\title{
Orthogonal Protecting Groups in the Synthesis of Tryptophanyl-Hexahydropyrroloindoles
}

\author{
Pau Ruiz-Sanchis, ${ }^{[\mathrm{a}, \mathrm{b}]}$ Svetlana A. Savina, ${ }^{[\mathrm{a}, \mathrm{c}]}$ Gerardo A. Acosta, ${ }^{[\mathrm{a}, \mathrm{c}]}$ \\ Fernando Albericio, ${ }^{*[a, b, d]}$ and Mercedes Álvarez ${ }^{*[a, b, c]}$
}

Keywords: Natural products / Heterocycles / Amino acids / Protecting groups

The synthesis of various polycyclic systems containing a $C^{3 a}-N^{i}$ bond between a hexahydropyrrolo[2,3-b]indole and an indole tryptophan is described here. A series of experiments was run to determine the best combination of five orthogonal protecting groups and the best reaction conditions for formation of said bond, which is a common feature among many recently discovered marine natural products.

\section{Introduction}

The tricyclic motif hexahydropyrrolo[2,3-b]indole (HPI) is present in many natural compounds with important bioactivities. ${ }^{[1]}$ These compounds all feature a substituent at the 3a-position of the HPI, such as a methyl group, in (-)-physostigmine, ${ }^{[2]}$ a prenyl, in flustramines, ${ }^{[3]}$ brevicompanines ${ }^{[4]}$ and roquefortines; ${ }^{[5]}$ and a newly discovered HPI linked by one aromatic carbon, in idiospermuline, ${ }^{[6]}$ psychotridine ${ }^{[7]}$ and quadrigemine. ${ }^{[8]}$ Recently isolated natural compounds such as psychotrimine, ${ }^{[9]}$ chaetomin and the chaetocochins ${ }^{[10]}$ contain an unusual bond between the $3 \mathrm{a}-$ position of the HPI and the indole nitrogen of either a tryptamine or a tryptophan (Figure 1). Kapakahines are natural products with a bond between the $C^{4 a}$ of an $\alpha$-carboline and the indole nitrogen of an $N$-Trp. ${ }^{[11]}$

[a] Institute for Research in Biomedicine, Barcelona Science Park, Baldiri Reixac 10, 08028 Barcelona, Spain.

[b] Laboratory of Organic Chemistry, Faculty of Pharmacy, University of Barcelona, 08028 Barcelona, Spain.

[c] CIBER-BBN, Networking Center on Bioengineering, Biomaterials and Nanomedicine, Barcelona Science Park, Baldiri Reixac 10 08028 Barcelona, Spain.

[d] Department of Organic Chemistry, University of Barcelona, 08028 Barcelona, Spain.

albericio@irbbarcelona.org, mercedes.alvarez@irbbarcelona.org Supporting information for this article is available on the WWW under http://www.eurjoc.org/ or from the author.
To date, four total syntheses of psychotrimine have been reported. ${ }^{[12]}$ Takayama et al. were the first to synthesize this compound, ${ }^{[12 a]}$ assembling the HPI motif from a phenylacetonitrile that contains an indoline at the appropriate $\alpha$-nitrile position. In contrast, Newhouse and Baran $^{[12 b]}$ prepared psychotrimine via simultaneous formation of the HPI and the $\mathrm{N}-\mathrm{C}^{3 a}$ bond. They later employed the same strategy to prepare kapakahines B and $\mathrm{F}^{[13]}$, and (+)-psychotetramine. ${ }^{[14]}$

During the course of the present work, Rainier et al. published a study on $\mathrm{N}-\mathrm{C}^{3 a}$ bond formation via bromo-displacement of 3abromo-HPIC with the $N$-anion of indole. ${ }^{[15]}$ The same group harnessed this chemistry to prepare kapakahines $\mathrm{E}$ and $\mathrm{F}^{[16]}$ and more recently, described a mechanism for the substitution. ${ }^{[17]}$

Compound 1, which contains a bond between the $C^{3 a}$ of HPI and the $N$ of an indole, could be used as a scaffold for the synthesis of many natural products and analogs. In the work reported here, 1 was synthesized via nucleophilic substitution of the bromine at position $3 \mathrm{a}$ of $3 \mathrm{a}$-bromo-HPI with an $\mathrm{N}$-indole anion (Figure 2). To ensure chemoselectivity during this chemistry, five orthogonal protecting groups were required. Studies to determine the best protecting groups and conditions for this bond formation were then performed and are described herein.

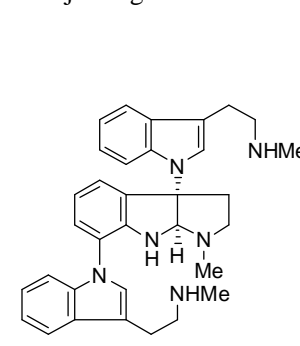

Psychotrimine

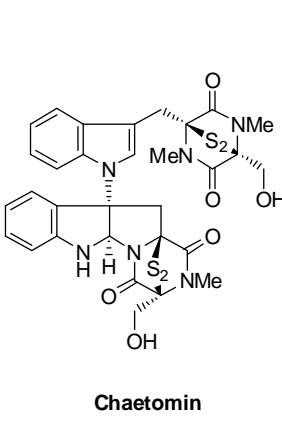

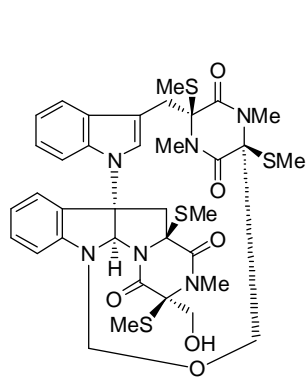

Chaetocochin A

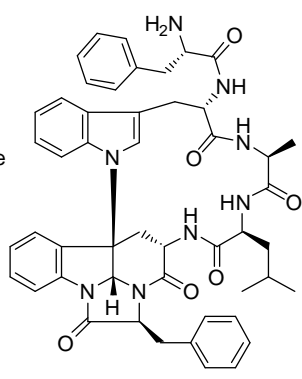

Kapakahine B

Figure 1. Natural products containing a bond between the $C^{3 a}$ of an HPI, or the $C^{4 a}$ of an $\alpha$-carboline, and the indole nitrogen of either a tryptamine or a tryptophan. 

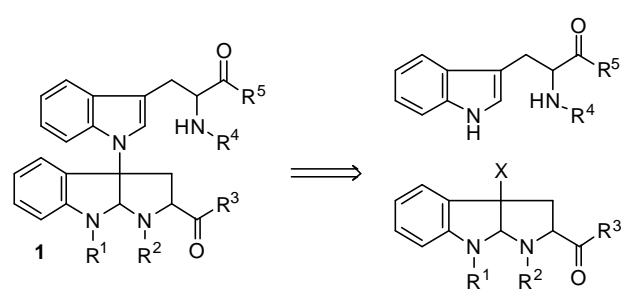

Figure 2. Retrosynthesis of compound $\mathbf{1}$.

\section{Results and Discussion}

First of all, various bromo analogs of 3a-bromo-1,2,3,3a,8,8ahexahydropyrrolo[2,3-b]indole-2-carboxylate (3a-Br-HPI; 3) were synthesized using two different procedures, which were subsequently compared for performance (Table 1). The first one follows the route described by Taniguchi and Hino, ${ }^{[18]}$ based on cyclization of a protected Trp in acidic medium, followed by aniline protection and subsequent benzylic bromination of HPI-2carboxylate. The second procedure is an one-step brominationcyclization of a totally protected Trp using $N$-bromosuccinimide (NBS) and pyridinium $p$-toluenesulfonate (PPTS) ${ }^{[19]}$ The resulting products $\mathbf{3}$ and their stereochemistries (endo/exo) are listed in Table 1. Although a three (two amino and one carboxylic protecting groups) orthogonal systems is desirable, the use of the same amino protecting groups $\left(\mathrm{R}^{1}=\mathrm{R}^{2}\right)$ for both amino groups were also studied (Table 1, Entries 2, 3, and 16) having in mind the different nucleophilicity of both amino functions. For the carboxylic protection, common esters such as methyl, $t$-butyl, and allyl were tested. On the other hand, for the amino function both alkoxycarbonyl, i.e., tert-butoxycarbonyl (Boc), allyloxycarbonyl (Alloc), benzyloxycarbonyl (Cbz), 2,2,2-trichloroetoxycarbonyl (Troc), and metoxycarbonyl (Moc), and and sulfonyl, i.e., 2nitrobenzenesulfonyl (Nosyl) and $\mathrm{SO}_{2} \mathrm{Ph}$ were tested. Additionally, , protected amino acids $\left(\mathrm{R}^{2}\right.$ and $\mathrm{R}^{3}$ Table 1 , Entries 14-16) were assayed with the idea of studying the size and/or the electronic properties of protecting groups.

The overall transformation of L-Trp-OMe (the starting material) into 3 was highly demanding, as illustrated by the yields, which ranged from poor to moderate. Method $\mathrm{B}$, which is shorter, gave better yields for the same set of protecting groups (Table 1: Entries 7, 12 and 13) and has the important additional advantage of being amenable to use of various protecting groups for the $\alpha$-amino group $\left(\mathrm{R}^{2}\right)$. Cyclization with $\mathrm{H}_{3} \mathrm{PO}_{4}$ (Method $\mathrm{A}$ ) gave better yields when methoxycarbonyl $\left(\mathrm{R}^{2}=\mathrm{Moc}\right)$ was used as $N^{\alpha}$-Trp protecting group compared to when trichloroethoxycarbonyl $\left(\mathrm{R}^{2}=\right.$ Troc $)$ was used (see Table 1: Entries 12 and 7, respectively).

Despite various attempts in diverse conditions, we were unable to remove the Moc group from the $N^{I}$ of HPI-2-carboxylate. ${ }^{[21]}$ Furthermore, to the best of our knowledge, ${ }^{[1]}$ to date there have not been any reports of the Moc group being removed from the $N^{8}$ of HPI-2-carboxylate; instead, this group typically is reduced to obtain the desired $N$-Me product. ${ }^{[12 b, 22]}$

Compounds $\mathbf{3 b}$ and $3 \mathbf{c}$ possess two Boc groups at positions $N^{l}$ and $N^{8}$ which could be cleaved simultaneously; however, the amine of $N^{l}$ is more reactive than the aniline of $N^{8}$, which enabled chemoselective acylation of $N^{l}$ as reported by Danishefsky et al. ${ }^{[23]}$

The ${ }^{1} \mathrm{H}-\mathrm{NMR}$ signals corresponding to the protecting groups of $\mathrm{R}^{2}$-namely, the signals for the $\mathrm{CH}_{2}$ of $\mathrm{Cbz}$ or Troc, and for the $\mathrm{CH}_{3}$ of Moc-are broad or split, because the protons are diastereotopic.

The difference in stereochemistry of the products $\mathbf{3}$ obtained from each method is noteworthy. Comparison of the ${ }^{1} \mathrm{H}-\mathrm{NMR}$ spectra of the products $\mathbf{3 g}$ obtained from Method A and from Method B revealed significant differences in the signals for the proton at position 2 ( $\delta 4.67$ vs. $3.98 \mathrm{ppm}$, respectively) and for the methyl ester ( $\delta 3.21$ vs. 3.74 ppm, respectively). Based on these data, the stereochemistry of the product from Method A was

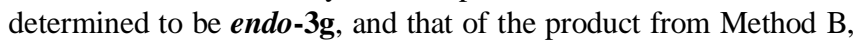
exo-3g (see Figure 3). The diamagnetic anisotropy of the phenyl ring shields the endo-methyl group $(\delta 3.21 \mathrm{ppm})$ and the exo-H2 $(\delta$ $3.98 \mathrm{ppm}) .{ }^{[24]}$ The same phenomenon occurred with the endo/exo $\mathbf{3 l}$ and $\mathbf{3 m}$ obtained with the appropriate method (see Supporting Information).
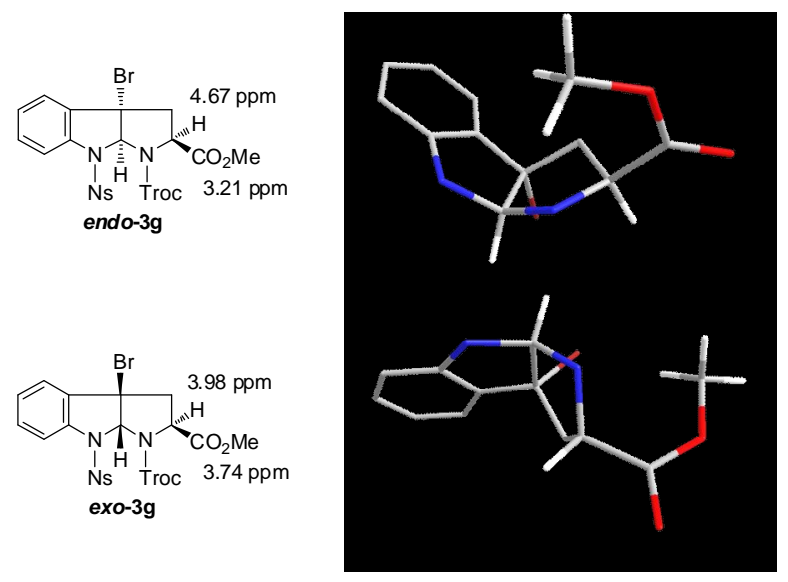

Figure 3. Comparison of the ${ }^{1} \mathrm{H}-\mathrm{NMR}$ data for endo and exo $\mathbf{3 g}$ (left). Three-dimensional models of the corresponding tricyclic systems (right). ${ }^{[25]}$

Compounds 3a, 3b, 3d, 3k, 3n-3p (Table 1: Entries 1, 2, 4, 11, 14-16) only showed one diastereomer on NMR data. Their stereochemistry assignments were determined by comparing the chemical shifts of proton and substituent at $C^{2}$ of HPI.

To obtain a more versatile intermediate during synthesis of $\mathbf{3 n}$, 3o and 3p via Method B, protected Ala or Ile were used as $N^{\alpha}$ - and $O$-protecting groups respectively. However, the synthesis of compound 6 required an indirect route (see Table 1, footnote d), because subjecting dipeptide 4 , which was $N^{\alpha}$-Alloc-Ala protected, to the acidic conditions for cyclization furnished the dimer $\mathbf{5}$ (Figure 4). Formation of $\mathbf{5}$ could be explained based on electrophilic substitution between $\mathbf{4}$ and the indoline that had formed after its protonation. 
Table 1. Synthesis of the bromo compounds 3a-p.

\begin{tabular}{|c|c|c|c|c|c|c|}
\hline \multirow{2}{*}{ \# } & & \multicolumn{2}{|c|}{$\begin{array}{l}\text { Method } \mathrm{A} \\
\text { 1. } \mathrm{H}_{3} \mathrm{PO}_{4}\left(\mathrm{R}^{1}=\mathrm{H}\right) \\
\text { 2. Protection of } \mathrm{N}^{8}\end{array}$} & 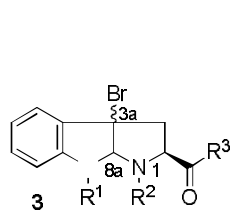 & \multirow{2}{*}{ endo:exo } \\
\hline & Comp. & $R^{\prime}$ & $\mathrm{R}^{2}$ & $\mathrm{R}^{\prime}$ & Method (Yield \%) & \\
\hline 1 & $3 a$ & Boc & Alloc & $\mathrm{OMe}$ & B (83) & exo \\
\hline 2 & $3 b$ & Boc & Boc & OAllyl & $B(30)^{[a]}$ & exo \\
\hline 3 & $3 c$ & Boc & Boc & $\mathrm{OMe}$ & B (86) & $4: 96^{[b]}$ \\
\hline 4 & $3 \mathrm{~d}$ & Boc & $\mathrm{Cbz}$ & $\mathrm{OMe}$ & B (78) & exo \\
\hline 5 & $3 e$ & Boc & Troc & $\mathrm{OMe}$ & B (77) & $11: 89^{[c]}$ \\
\hline 6 & $3 \mathrm{f}$ & Nosyl & $\mathrm{Cbz}$ & OtBu & B (80) & $7: 93^{[c]}$ \\
\hline \multirow{2}{*}{7} & \multirow{2}{*}{$3 \mathrm{~g}$} & \multirow{2}{*}{ Nosyl } & \multirow{2}{*}{ Troc } & \multirow{2}{*}{$\mathrm{OMe}$} & A (9) & endo \\
\hline & & & & & B (57) & $8: 92^{[\mathrm{c}]}$ \\
\hline 8 & $3 \mathrm{~h}$ & Nosyl & Troc & $\mathrm{OtBu}$ & B (59) & $6: 94^{[b]}$ \\
\hline 9 & $3 \mathrm{i}$ & $\mathrm{SO}_{2} \mathrm{Ph}$ & Boc & $\mathrm{OMe}$ & B (92) & $7: 93^{[b]}$ \\
\hline 10 & $3 j$ & $\mathrm{SO}_{2} \mathrm{Ph}$ & $\mathrm{Cbz}$ & $\mathrm{OMe}$ & B (82) & $4: 96^{[\mathrm{b}]}$ \\
\hline 11 & $3 \mathrm{k}$ & $\mathrm{SO}_{2} \mathrm{Ph}$ & $\mathrm{Cbz}$ & $\mathrm{OtBu}$ & B (83) & exo \\
\hline \multirow{2}{*}{12} & \multirow{2}{*}{31} & \multirow{2}{*}{$\mathrm{SO}_{2} \mathrm{Ph}$} & \multirow{2}{*}{ Moc } & \multirow{2}{*}{$\mathrm{OMe}$} & A (37) & endo \\
\hline & & & & & B (96) & $5: 95^{[b]}$ \\
\hline \multirow{2}{*}{13} & \multirow{2}{*}{$3 \mathrm{~m}$} & \multirow{2}{*}{$\mathrm{SO}_{2} \mathrm{Ph}$} & \multirow{2}{*}{ Moc } & \multirow{2}{*}{$\mathrm{O} t \mathrm{Bu}$} & A (28) & $91: 9^{[\mathrm{c}]}$ \\
\hline & & & & & B (58) & $25: 75^{[\mathrm{cc}]}$ \\
\hline 14 & $3 n$ & Boc & $N^{\alpha}$-Alloc-Ala & $\mathrm{OMe}$ & B (47) & exo \\
\hline 15 & 30 & Boc & Alloc & Ile-OMOM & B $(47)^{[\mathrm{d}]}$ & exo \\
\hline 16 & $3 p$ & Boc & Boc & Ile-OAllyl & $\mathrm{B}(41)^{[\mathrm{d}]}$ & exo \\
\hline
\end{tabular}

[a] 3b, 3o and 3p were synthesized from 3c, 3a and 3c, respectively, after hydrolysis and subsequent esterification or coupling with the protected Ile (see Supporting Information). [b] Ratio determined by HPLC. ${ }^{[20]}$ [c] Ratio determined by ${ }^{1} \mathrm{H}-\mathrm{NMR}$. [d]

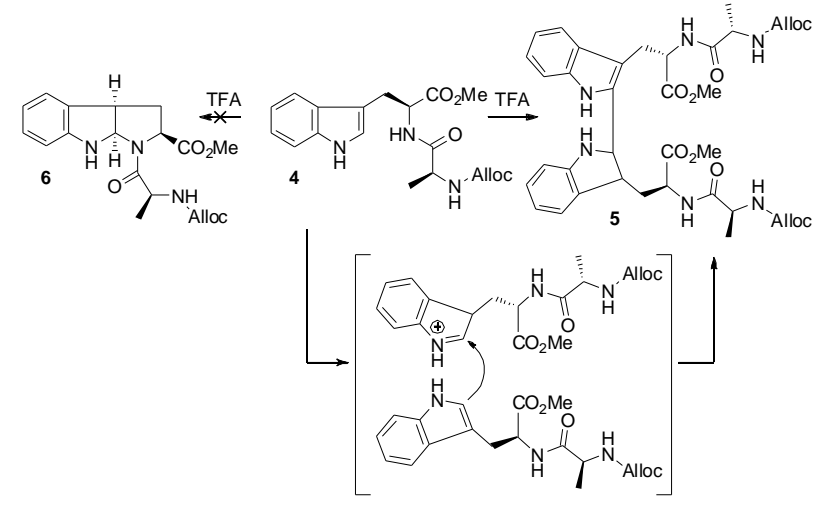

Figure 4. Dimerization of $\mathbf{4}$ under acidic conditions.

Consequently, in the first step of the HPI formation in Method A, working with an $N^{\alpha}$-carbamate protecting group at this position, instead an amide bond, is rather important.

The second part of this work comprised formation of the bond between the $C^{3 a}$ of the HPI and the $N^{i}$ of the Trp. Several pairs of base and solvent were tested to generate the indole anion that would drive the substitution to give compound $1 .^{[26]}$ The best conditions found comprised $\mathrm{NaH}$ in DMF at $70{ }^{\circ} \mathrm{C}$ for $1.5 \mathrm{~h}$. Every bromo derivative (3a-p) was tested with several protected Trp's. A distinguishing data point in the ${ }^{13} \mathrm{C}-\mathrm{NMR}$ data for compounds $\mathbf{1}$ and $\mathbf{3}$ is the chemical shift of the quaternary $C^{3 a}$, which is less shielded in $\mathbf{1}$ ( $\delta 72.4$ to $82.2 \mathrm{ppm})$ than in $\mathbf{3}(\delta 53.7$ to $67.9 \mathrm{ppm})$. The results of these substitutions are summarized in Table 2 .
The best yields of $\mathbf{1}$ in the nucleophilic substitution were found using 3a, 31, 3m and 3p (Table 2: Entries 1 and 7-10, respectively). Moderate yields were obtained for the substitutions with bromides 3c, 3d and 3g (Entries 3-6). However, very poor yields $(<10 \%$, data not shown) were observed when bromides $\mathbf{3 b}, \mathbf{3 e}, \mathbf{3 f}, \mathbf{3 h}-\mathbf{k}$ and $3 \mathrm{n}$ were reacted with different protected versions of $\mathbf{7}$, which contains two additional protecting groups.

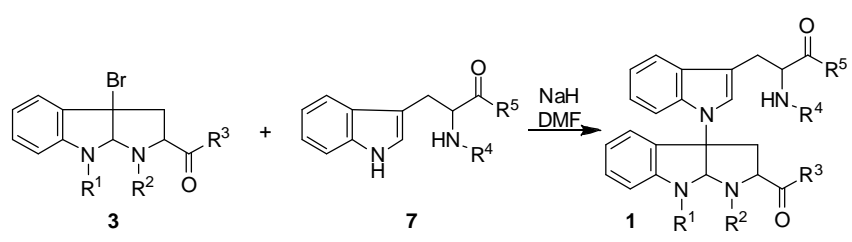

Table 2. Nucleophilic substitution at the C3a of 3a-Br-HPI

\begin{tabular}{cccccccc}
\hline$\#$ & $3^{[a]}$ & $\mathrm{R}^{4}$ & $\mathrm{R}^{5}$ & $3^{[\mathrm{b}]}$ & $7^{[\mathrm{b}]}$ & $1^{[\mathrm{b}]}$ & $\begin{array}{c}\text { Comp } \\
(\%)^{[\mathrm{c}]}\end{array}$ \\
\hline 1 & exo-3a & Phth & OMe & 39 & 18 & 43 & $\mathbf{1 a}(41)$ \\
2 & $\mathbf{3 c}$ & Moc & OMe & 49 & 22 & 29 & $\mathbf{1 b}(20)$ \\
3 & $\mathbf{3 c}$ & Phth & OMe & 66 & 11 & 24 & $\mathbf{1 c}(21)$ \\
4 & exo-3d & Phth & OMe & 28 & 14 & 58 & $\mathbf{1 d}(26)$ \\
5 & endo-3g & Alloc & OMe & 24 & 21 & 48 & $\mathbf{1 e}(29)$ \\
6 & exo-3g & Alloc & OtBu & 62 & 22 & 15 & $\mathbf{1 f}(22)$ \\
7 & endo-31 & Alloc-Ile & OtBu & - & 24 & 50 & $\mathbf{1 g}(41)$ \\
8 & endo-3l & Moc & OMe & - & 1 & 91 & $\mathbf{1 h}(77)$ \\
9 & endo-3m & Boc-Ile & OAllyl & 29 & 20 & 48 & $\mathbf{1 i}(30)$ \\
10 & $\mathbf{3 p}$ & Phth & OMe & 13 & 42 & 11 & $\mathbf{1 j}(30)$ \\
\hline
\end{tabular}

[a] See Table 1 for the protecting groups used in each compound 3. [b] Percentage of each compound in the reaction crude (as measured by HPLC) ${ }^{[20]}$. $[\mathrm{c}]$ Yield of isolated compound. 
The phthalamide (Phth) was introduced as $\mathrm{R}^{4}$ because it is orthogonal to all protecting groups used in the first reactions (Table 2: Entries 1, 3, 4 and 10), as it eliminates all the $N^{\alpha}$ acid protons in 7. The wide range of yields in the resulting substitution (from 21 to $41 \%$ ) demonstrates the importance of the protecting groups in the starting bromide. The bromides $\mathbf{3 I}$ and $\mathbf{3 m}$ have the same protecting groups in both amino groups of HPI $\left(\mathrm{R}^{1}=\mathrm{SO}_{2} \mathrm{Ph}, \mathrm{R}^{2}=\right.$ Moc). Interestingly, the yield was lower when the group at $\mathrm{R}^{5}$ was tert-butyl ester (1g, Entry 7) compared to methyl ester (1h, Entry 8). Likewise, the yield was lower when $\mathrm{R}^{3}$ was tert-butyl ester (1i, Entry 9) compared to methyl ester (1h, Entry 8). The same trend was observed for 1f (Entry 6) and 1e (Entry 5), although to a lesser extent: the tert-butyl in $\mathbf{1 f}$ is more sterically hindered than the methyl ester in 1e. The results obtained with a protected Ile-Trp dipeptide as nucleophile (Entries 7 and 9), and with a Br-HPI and a protected Ile (Entry 10), are interesting, as they can serve as the stepping stone to synthesis of peptides found in natural compounds. Additionally, owing to this Ile protection, $\mathbf{1 j}\left(\mathrm{R}^{3}=\right.$ Ile-OAllyl, Entry 10) was obtained in higher yield than was $1 c\left(R^{3}=\right.$ OMe, Entry 3$)$, whose protecting groups are the same, except for at $R^{3}$.

Reaction of bromide 3n and $N^{\alpha}$-Phth-Trp-OMe unexpectedly gave compound $\mathbf{8}$. The product was characterized by mono- and bidimensional NMR and by HRMS (see Supporting Information). Important spectroscopy data for compound $\mathbf{8}$ are the lack of $\mathrm{Br}$, the $\alpha$-proton of the Trp, and the fact that the two protons of the cyclopropane $\mathrm{CH}_{2}(2 \mathrm{~d}, J=15.4 \mathrm{~Hz}$, at $\delta 3.43$ and $3.91 \mathrm{ppm})$ only exhibit a geminal coupling constant. The significant difference in the chemical shift of the $\alpha$-proton of the Ala in $3 \mathbf{n}(\delta 5.02 \mathrm{ppm})$ and that of the Ala in $\mathbf{8}(\delta 4.11 \mathrm{ppm})$ could be justified by the different electronic effects in each compound. One hypothetical mechanism for formation of $\mathbf{8}$ begins with deprotonation of the $C^{2}$ of the HPI, made possible by the basic conditions, followed by intramolecular bromine displacement and subsequent formation of cyclopropane, to afford intermediate $\mathbf{B}$ (see Figure 5). The high strain in $\mathbf{B}$ could drive opening of the aminal and subsequent cyclization, to give a more relaxed cyclohexane (see Figure 5).

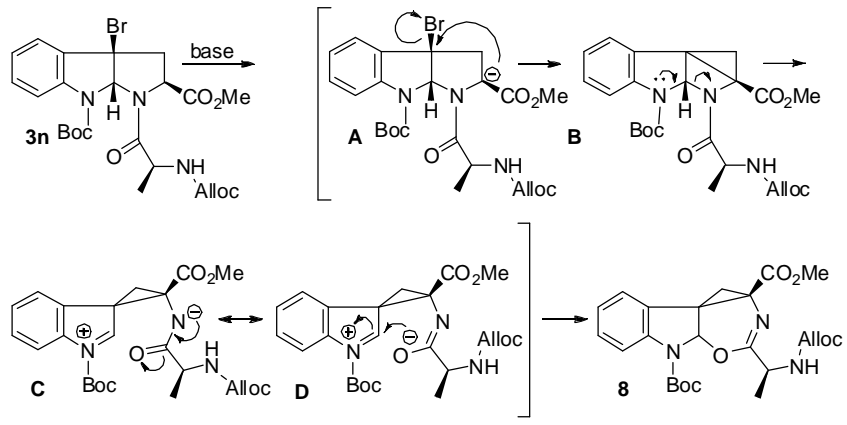

Figure 5. Hypothetical mechanism for formation of $\mathbf{8}$.

This is the first time that the authors of this paper have isolated a compound such as $\mathbf{8}$ after the nucleophilic substitution reaction with the aforementioned conditions. Recently, J.D. Rainier et al. have reported the behavior of $\mathbf{3 c}$ under basic conditions of $\mathrm{KO} t \mathrm{Bu}$ and have isolated a tetracycle-containing compound that resembles B. ${ }^{[27]}$

\section{Conclusions}

In conclusion, various analogs of $3 \mathrm{a}$-bromo-1,2,3,4,4a,8,8ahexahydropyrrolo[2,3-b]indole-2-carboxylate (3), protected with different combinations of three orthogonal protecting groups, were prepared by two different routes. The routes were then compared for performance. Method A, based on sequential cyclization, protection and bromination, provided the thermodynamic endocompound; whereas Method $\mathrm{B}$, based on one pot brominationcyclization of a fully protected Trp, afforded mainly the kinetic exo-bromide. The influence of the protecting groups on formation of the $\mathrm{N}-\mathrm{C}^{3 a}$ bond between the Trp and HPI to give compounds 1f, $\mathbf{1 g}$, and 1i (containing five orthogonal protecting groups) and compounds 1a, 1d, $\mathbf{1 e}$ and $\mathbf{1 j}$ (containing four orthogonal protecting groups) also was evaluated. Some of these compounds contain a protected Ile as the $\mathrm{R}^{4}$ to protect the $\alpha$-amino Trp; the orthogonal protecting groups enable synthetic versatility for constructing more structurally complex molecules. The protecting groups in the bromides $\mathbf{3}$ determined the yields of compounds 1a, 1c, $1 d$ and $\mathbf{1 j}$, whose starting $N^{\alpha}$-Phth-Trp-OMe 7 is the same. Moreover, the importance of the carbamate protecting group at $\mathrm{R}^{2}$ should be emphasized: unexpectedly, compound $\mathbf{5}$ was obtained from an attempted cyclization of $\mathbf{4}$ in acidic medium (using an Ala amide bond for protecting the $\mathrm{N}$ in 4 ) and compound $\mathbf{8}$ was obtained from an attempted nucleophilic substitution of the bromine at $\mathrm{C}^{3 \mathrm{a}}$ of $\mathbf{3 n}$.

\section{Experimental Section}

General procedure for the synthesis of 1: A solution of $6(3.0 \mathrm{mmol})$ in dry DMF $(10 \mathrm{~mL})$ was added to a suspension of $60 \% \mathrm{NaH}$ in mineral oil (1.2 eq.) in dry DMF (20 mL), and the resulting mixture was stirred at room temperature for $15 \mathrm{~min}$. A solution of $3(3.0 \mathrm{mmol})$ in dry DMF $(10 \mathrm{~mL})$ was then added. The mixture was stirred at $70{ }^{\circ} \mathrm{C}$ for $1.5 \mathrm{~h}$. The reaction mixture was then cooled to room temperature and quenched with $\mathrm{H}_{2} \mathrm{O}$. The aqueous phase was saturated with $\mathrm{NaCl}$ and extracted with EtOAc. The organic solution was dried over anhyd. $\mathrm{Na}_{2} \mathrm{SO}_{4}$. The solvent was removed, and the residue was purified by column chromatography on silica gel to afford 1 .

Compound 1a: Purified by flash chromatography (hexane/EtOAc, from 90:10 to 50:50), endo:exo (57:43) mixture. ${ }^{1} \mathrm{H}-\mathrm{NMR}\left(400 \mathrm{MHz}, \mathrm{CDCl}_{3}\right)$ : $\delta$ (ppm) 1.48 and $1.49(2 \mathrm{~s}, 9 \mathrm{H}) ; 2.82$ and $2.92(2 \mathrm{~d}, J=13.0 \mathrm{~Hz}, 1 \mathrm{H}) ; 3.17$ and $3.21(2 \mathrm{~s}, 3 \mathrm{H}) ; 3.33-3.45(\mathrm{~m}, 1 \mathrm{H}) ; 3.49-3.68(\mathrm{~m}, 2 \mathrm{H}) ; 3.76(\mathrm{~s}, 3 \mathrm{H}) ; 4.59$ $4.74(\mathrm{~m}, 2 \mathrm{H}) ; 4.88(\mathrm{t}, J=9.8 \mathrm{~Hz}, 1 \mathrm{H}) ; 5.08-5.16(\mathrm{~m}, 1 \mathrm{H}) ; 5.17-5.31(\mathrm{~m}$, $2 \mathrm{H}) ; 5.85-5.99(\mathrm{~m}, 1 \mathrm{H}) ; 6.62-6.88(\mathrm{~m}, 3 \mathrm{H}) ; 6.95-7.14(\mathrm{~m}, 4 \mathrm{H}) ; 7.30$ (dd, $J$ $=7.4$ and $14.8 \mathrm{~Hz}, 1 \mathrm{H}) ; 7.57(\mathrm{t}, J=6.8 \mathrm{~Hz}, 1 \mathrm{H}) ; 7.68(\mathrm{dd}, J=3.1$ and 5.5 $\mathrm{Hz}, 1 \mathrm{H}) ; 7.71(\mathrm{dd}, J=3.0$ and $5.6 \mathrm{~Hz}, 1 \mathrm{H}) ; 7.73-7.79(\mathrm{~m}, 3 \mathrm{H}) .{ }^{13} \mathrm{C}-\mathrm{NMR}$ $\left(100 \mathrm{MHz}, \mathrm{CDCl}_{3}\right): \delta(\mathrm{ppm}) 24.4(\mathrm{t}) ; 28.1(3 \mathrm{q}) ; 38.1(\mathrm{t}) ; 52.2(\mathrm{q}) ; 52.3(\mathrm{~d}) ;$ 52.5 and $52.8(\mathrm{q}) ; 53.4(\mathrm{~s}) ; 59.3$ and $59.4(\mathrm{~d}) ; 66.6(\mathrm{t}) ; 79.6(\mathrm{~d}) ; 82.2(2 \mathrm{~s})$; $110.6(\mathrm{~s}) ; 111.2$ and 111.3 (d); 117.6 and $117.7(\mathrm{t}) ; 119.2$ and $119.3(\mathrm{~d})$; 120.0 (d); 122.3 and 122.4 (d); 123.4 (4d); 124.4 and 124.7 (d); 129.7 (s); 129.8 (s); 130.9 (d); 131.6 (s); 131.7 (s); 132.5 (d); 134.0 (3d); 134.7 (s); 143.3 (s); 143.4 (s); 151.8 (s); 151.9 (s); 167.2 (s); 167.4 (s); 169.4 (s); $170.6(\mathrm{~s})$. IR (KBr): $v\left(\mathrm{~cm}^{-1}\right) 2952,1716,1390,1255,1158,1019,721$. HRMS (ESI) calculated for $\mathrm{C}_{41} \mathrm{H}_{40} \mathrm{~N}_{4} \mathrm{O}_{10} \mathrm{Na} \mathrm{m} / z\left(\mathrm{M}+\mathrm{Na}^{+}\right) 771.2642$, found 771.2634

Compound 1b: Purified by flash chromatography (hexane/EtOAc, from 80:20 to 50:50). ${ }^{1} \mathrm{H}-\mathrm{RMN}\left(400 \mathrm{MHz}, \mathrm{CDCl}_{3}\right): \delta(\mathrm{ppm}) 1.50(\mathrm{~s}, 9 \mathrm{~h}) ; 1.52(\mathrm{~s}$, 9H); 2.98-3.20 (m, 3H); $3.23(\mathrm{~s}, 3 \mathrm{H}) ; 3.54-3.65(\mathrm{~m}, 7 \mathrm{H}) ; 4.60(\mathrm{~m}, 1 \mathrm{H}) ; 4.90$ (bs, 1H); $5.19(\mathrm{t}, J=8.3 \mathrm{~Hz}, 1 \mathrm{H}) ; 6.69(\mathrm{~d}, J=7.3 \mathrm{~Hz}, 1 \mathrm{H}) ; 6.75(\mathrm{~s}, 1 \mathrm{H})$; 7.07-7.33 (m, 5H); 7.35-7.42 (m, 1H); 7.52 (t, $J=7.5 \mathrm{~Hz}, 1 \mathrm{H}) ; 7.67$ (bs, 1H). ${ }^{13} \mathrm{C}-\mathrm{RMN}\left(100 \mathrm{MHz}, \mathrm{CDCl}_{3}\right): \delta(\mathrm{ppm}) 27.6(\mathrm{t}) ; 28.2(3 \mathrm{q}) ; 28.3(3 \mathrm{q})$; 38.4 (t); 52.2 (q); 52.3 (q); 52.4 (q); 54.5 (d); 59.4 (d); 72.5 (s); 79.8 (d); 81.6 (s); 82.4 (s); 109.3 (s); 111.6 (d); 119.4 (d); 120.2 (2d); 122.5 (d); $123.6(\mathrm{~d}) ; 124.9$ (d); $125.3(\mathrm{~d}) ; 130.2(\mathrm{~s}) ; 131.0(\mathrm{~d}) ; 131.1(\mathrm{~s}) ; 134.8(\mathrm{~s})$; 143.5 (s); 152.2 (s); 156.6 (s); 164.3 (s); 171.5 (s); 172.3 (s). IR (KBr): v $\left(\mathrm{cm}^{-1}\right)$ 3352, 2978, 1719, 1394, 1368, 1158, 740. HRMS (ESI+) calculated for $\mathrm{C}_{36} \mathrm{H}_{45} \mathrm{~N}_{4} \mathrm{O}_{10} \mathrm{~m} / z\left(\mathrm{M}+\mathrm{H}^{+}\right)$693.3130, found 693.3118. 
Compound 1c: Purified by flash chromatography $\left(\mathrm{MeCN} / \mathrm{H}_{2} \mathrm{O}\right.$, from 30:70 to $90: 10) .{ }^{1} \mathrm{H}-\mathrm{RMN}\left(400 \mathrm{MHz}, \mathrm{CDCl}_{3}\right): \delta(\mathrm{ppm}) 1.46$ and $1.48(2 \mathrm{~s}, 9 \mathrm{H})$; 1.50 and $1.52(2 \mathrm{~s}, 9 \mathrm{H}) ; 2.77$ and $2.90(2 \mathrm{~d}, J=12.9 \mathrm{~Hz}, 1 \mathrm{H}) ; 3.17$ and 3.21 $(2 \mathrm{~s}, 3 \mathrm{H}) ; 3.38(\mathrm{dd}, J=9.3$ and $12.9 \mathrm{~Hz}, 1 \mathrm{H}) ; 3.50-3.66(\mathrm{~m}, 2 \mathrm{H}) ; 3.76(\mathrm{~s}$, $3 \mathrm{H}) ; 4.83(\mathrm{bs}, 1 \mathrm{H}) ; 5.08-5.18(\mathrm{~m}, 1 \mathrm{H}) ; 6.64-6.75(\mathrm{~m}, 3 \mathrm{H}) ; 6.82(\mathrm{t}, J=7.6$ $\mathrm{Hz}, 1 \mathrm{H}) ; 7.03-7.14(\mathrm{~m}, 4 \mathrm{H}) ; 7.24-7.31(\mathrm{~m}, 1 \mathrm{H}) ; 7.57(\mathrm{dd}, J=7.6$ and 7.8 $\mathrm{Hz}, 1 \mathrm{H}) ; 7.66-7.79$ (m, 4H). ${ }^{13} \mathrm{C}-\mathrm{RMN}\left(100 \mathrm{MHz}, \mathrm{CDCl}_{3}\right): \delta(\mathrm{ppm}) 24.4(\mathrm{t})$; $28.2(3 \mathrm{q}) ; 28.3(3 \mathrm{q}) ; 38.8(\mathrm{t}) ; 52.1(\mathrm{q}) ; 52.6(\mathrm{~d}) ; 52.8(\mathrm{q}) ; 59.3(\mathrm{~d}) ; 72.4(\mathrm{~s}) ;$ 79.6 (d); 79.7 (s); 82.0 (s); 110.4 (s); 111.4 (d); 119.2 (d); 119.9 (d); 122.3 (d); 123.1 (d); 123.4 (2d); 124.4 (d); 124.7 (d); 125.0 (d); 128.9 (s); 129.7 (s); $130.8(\mathrm{~d}) ; 131.7(\mathrm{~s}) ; 134.0$ (s and 2d); 134.7 (s); 143.5 (s); $151.8(2 \mathrm{~s})$; 167.2 (s); 167.4 (s); 169.4 (s); 171.0 (s). IR (KBr): v ( $\left.\mathrm{cm}^{-1}\right)$ 2977, 1716, 1390, 1255, 1158, 1019, 739, 721. HRMS (ESI+) calculated for $\mathrm{C}_{42} \mathrm{H}_{45} \mathrm{~N}_{4} \mathrm{O}_{10} \mathrm{~m} / z\left(\mathrm{M}+\mathrm{H}^{+}\right)$765.3130, found 765.3091.

Compound 1d: Purified by flash chromatography (hexane/EtOAc, from 90:10 to 50:50), endo:exo (69:31) mixture. ${ }^{1} \mathrm{H}-\mathrm{NMR}\left(400 \mathrm{MHz}, \mathrm{CDCl}_{3}\right)$ : $\delta$ (ppm) $1.45(\mathrm{~s}, 9 \mathrm{H}) ; 2.80(\mathrm{~d}, J=13.0 \mathrm{~Hz}, 1 \mathrm{H}) ; 3.16$ and $3.20(2 \mathrm{~s}, 3 \mathrm{H}) ; 3.37$ $(\mathrm{dt}, J=9.3$ and $13.0 \mathrm{~Hz}, 1 \mathrm{H}) ; 3.49-3.68(\mathrm{~m}, 2 \mathrm{H}) ; 3.76(\mathrm{~s}, 3 \mathrm{H}) ; 4.88(\mathrm{t}, J=$ $10.6 \mathrm{~Hz}, 1 \mathrm{H}) ; 5.07-5.30(\mathrm{~m}, 3 \mathrm{H}) ; 6.62-6.89(\mathrm{~m}, 3 \mathrm{H}) ; 7.02-7.10(\mathrm{~m}, 4 \mathrm{H})$; 7.26-7.36 (m, 6H); 7.54-7.61 $(\mathrm{m}, 1 \mathrm{H}) ; 7.66(\mathrm{dd}, J=3.1$ and $5.5 \mathrm{~Hz}, 1 \mathrm{H})$; $7.70(\mathrm{dd}, J=3.1$ and $5.5 \mathrm{~Hz}, 1 \mathrm{H}) ; 7.74(\mathrm{dd}, J=3.1$ and $5.5 \mathrm{~Hz}, 1 \mathrm{H}) ; 7.76$ (dd, $J=3.1$ and $5.5 \mathrm{~Hz}, 2 \mathrm{H}) .{ }^{13} \mathrm{C}-\mathrm{NMR}\left(400 \mathrm{MHz} \mathrm{CDCl}_{3}\right): \delta(\mathrm{ppm}) 24.4$ $(\mathrm{t}) ; 28.1(3 \mathrm{q}) ; 38.3(\mathrm{t}) ; 52.2(\mathrm{q}) ; 52.5(\mathrm{~d}) ; 52.8(\mathrm{q}) ; 59.4(\mathrm{~d}) ; 67.5(\mathrm{t}) ; 72.4$ (s); 79.7 (d); 82.2 (s); 110.5 (s); 111.3 (d); 119.2 (d); 120.0 (d); 122.4 (2d); 123.4 (4d); 124.6 (d); 127.8 (d); 128.0 (d); 128.4 (3d); 129.7 (s); 130.9 (d); 131.6 (s); 134.0 (2d); 134.7 (s); 136.2 (s); 143.2 (s); 143.4 (s); 151.8 (s); 167.2 (s); 167.3 (s); 169.4 (s); 169.4 (s); 170.6 (s). IR (KBr): v ( $\left.\mathrm{cm}^{-1}\right) 2952$, 1716, 1389, 1255, 1158, 1020, 721. HRMS (ESI) calculated for $\mathrm{C}_{45} \mathrm{H}_{42} \mathrm{~N}_{4} \mathrm{O}_{10} \mathrm{Na} m / z\left(\mathrm{M}+\mathrm{Na}^{+}\right)$821.2799, found 821.2804.

Compound 1e: Purified by flash chromatography (hexane/EtOAc, from 70:30 to 60:40). ${ }^{1} \mathrm{H}-\mathrm{RMN}\left(500 \mathrm{MHz}, \mathrm{CDCl}_{3}\right): \delta(\mathrm{ppm})$ 2.81-2.95 (m, 1H); $2.98(\mathrm{~d}, J=13.4 \mathrm{~Hz}, 1 \mathrm{H}) ; 3.07$ (ddd, $J=5.0,5.4$ and $14.8 \mathrm{~Hz}, 1 \mathrm{H}) ; 3.27$ (s, $3 \mathrm{H}) ; 3.57$ and $3.63(2 \mathrm{~s}, 3 \mathrm{H}) ; 3.62-3.68(\mathrm{~m}, 1 \mathrm{H}) ; 4.06-4.17$ and $4.59-4.67$ $(2 \mathrm{~m}, 1 \mathrm{H}) ; 4.74-4.82$ and 5.27-5.33 $(2 \mathrm{~m}, 1 \mathrm{H}) ; 4.50-4.57(\mathrm{~m}, 3 \mathrm{H}) ; 4.96-5.08$ $(\mathrm{m}, 1 \mathrm{H}) ; 5.09-5.30(\mathrm{~m}, 3 \mathrm{H}) ; 5.80-5.96(\mathrm{~m}, 1 \mathrm{H}) ; 6.32$ and $6.43(2 \mathrm{~s}, 1 \mathrm{H})$; 6.80 and $6.93(2 \mathrm{~d}, J=13.7 \mathrm{~Hz}, 1 \mathrm{H}) ; 7.10(\mathrm{dd}, J=7.6$ and $8.1 \mathrm{~Hz}, 1 \mathrm{H})$; 7.12-7.18 (m, 2H); 7.21 (bs, 1H); 7.37 (bs, 1H); 7.39-7.49 (m, 4H); 7.52$7.59(\mathrm{~m}, 1 \mathrm{H}) ; 7.62(\mathrm{dd}, J=2.9$ and $8.1 \mathrm{~Hz}, 1 \mathrm{H}) ; 7.86(\mathrm{bs}, 1 \mathrm{H}) .{ }^{13} \mathrm{C}-\mathrm{RMN}$ $\left(125 \mathrm{MHz}, \mathrm{CDCl}_{3}\right): \delta(\mathrm{ppm}) 27.7$ and $27.8(\mathrm{t}) ; 37.9(\mathrm{t}) ; 52.3(\mathrm{q}) ; 52.6(\mathrm{q})$; 54.1 and $54.4(\mathrm{~d}) ; 59.4$ and $60.1(\mathrm{~d}) ; 65.8(\mathrm{t}) ; 72.9$ and $74.0(\mathrm{~s}) ; 74.6$ and $75.3(\mathrm{t}) ; 81.0$ and $81.7(\mathrm{~d}) ; 94.8$ and $95.3(\mathrm{~s}) ; 109.3(\mathrm{~s}) ; 110.9(\mathrm{~d}) ; 117.8$ and 117.9 (t); 119.7 (d); 119.8 (d); 120.5 (d); 122.8 (d); 124.0 and 124.2 (d); 124.8 (d); 125.8 (d); 126.2 (d); 126.5 (s); 129.7 (d); 130.2 (s); 130.5 (s); 131.5 (d); 132.1 (d); 132.6 (d); 133.3 (d); 133.8 and 133.9 (s); 143.0 and 143.1 (s); 147.3 (s); 151.6 and 152.6 (s); 155.4 and 155.5 (s); 169.9 (s); 171.9 and $172.0(\mathrm{~s})$. IR (KBr): $v\left(\mathrm{~cm}^{-1}\right) 3369,2953,1733,1545,1402,1368$, 1231, 1174, 1055, 852, 740, 580. HRMS (ESI+) calculated for $\mathrm{C}_{37} \mathrm{H}_{35} \mathrm{~N}_{5} \mathrm{O}_{12} \mathrm{SCl}_{3} \mathrm{~m} / z$. $\left(\mathrm{M}+\mathrm{H}^{+}\right)$878.1063, found 878.1059.

Compound 1f: Purified by flash chromatography $\left(\mathrm{MeCN} / \mathrm{H}_{2} \mathrm{O}\right.$, from 0:100 to $70: 30) .{ }^{1} \mathrm{H}-\mathrm{RMN}\left(400 \mathrm{MHz}, \mathrm{CDCl}_{3}\right): \delta$ (ppm) 1.22 and $1.34(2 \mathrm{~s}, 9 \mathrm{H})$; 2.74-3.11 (m, 3H); 3.27 (s, 3H); 3.49-3.68 (m, 1H); 4.45 (bs, 1H); 4.49$4.58(\mathrm{~m}, 2 \mathrm{H})$; 4.59-4.84 (m, 2H); 5.02 (bs, 1H); 5.09-5.33 (m, 3H); 5.79$5.96(\mathrm{~m}, 1 \mathrm{H}) ; 6.36$ and $6.46(2 \mathrm{~s}, 1 \mathrm{H}) ; 6.82$ and $6.94(2 \mathrm{~d}, J=18.7 \mathrm{~Hz}, 1 \mathrm{H})$; 7.04-7.25 (m, 4H); 7.29-7.58 (m, 6H); 7.62 (d, $J=8.3 \mathrm{~Hz}, 1 \mathrm{H}) ; 7.75-7.97$ (m, 1H). ${ }^{13} \mathrm{C}-\mathrm{RMN}\left(100 \mathrm{MHz}, \mathrm{CDCl}_{3}\right): \delta(\mathrm{ppm}) 27.8$ and $28.0(3 \mathrm{q}) ; 27.9$ $(\mathrm{t}) ; 37.9(\mathrm{t}) ; 52.6(\mathrm{q}) ; 54.4$ and $54.6(\mathrm{~d}) ; 59.4(\mathrm{~d}) ; 65.7(\mathrm{t}) ; 74.6(\mathrm{~s}) ; 75.3(\mathrm{t}) ;$ 81.6 (s); 82.2 (d); 109.5 (s); 110.8 (d); 117.8 (t); 119.8 (d); 120.2 (d); 120.5 (d); 122.7 (d); 124.2 (d); 124.6 (d); 125.8 (d); 126.3 (s); 126.7 (d); 129.7 (d); 130.5 (s); 131.7 (d); 132.1 (d); 132.7 (d); 133.3 (d); 133.7 (s); 137.9 (s); 143.1 (s); 147.4 (s); 155.6 (s); 155.7 (s); 169.9 (s); 170.6 (s). IR (KBr): v $\left(\mathrm{cm}^{-1}\right) 3419,2979,1733,1545,1368,1230,1173,1129,1055,740,581$ HRMS (ESI+) calculated for $\mathrm{C}_{40} \mathrm{H}_{41} \mathrm{~N}_{5} \mathrm{O}_{12} \mathrm{SCl}_{3} \mathrm{~m} / z\left(\mathrm{M}+\mathrm{H}^{+}\right) 920.1538$, found 920.1578
Compound 1g: Purified by flash chromatography (hexane/EtOAc, 60:40). ${ }^{1} \mathrm{H}-\mathrm{NMR}$ (400 MHz, $\mathrm{CDCl}_{3}$ ): $\delta$ (ppm) 0.81-0.88 (m, 6H); 1.00-1.12 (m, $1 \mathrm{H}) ; 1.31$ (s, 9H); 1.39 (bs, $1 \mathrm{H}) ; 1.72-1.82(\mathrm{~m}, 1 \mathrm{H}) ; 2.67$ (dd, $\mathrm{J}=4.7$ and $14.7 \mathrm{~Hz}, 1 \mathrm{H}) ; 2.82(\mathrm{~d}, \mathrm{~J}=13.3 \mathrm{~Hz}, 1 \mathrm{H}) ; 3.08(\mathrm{dd}, \mathrm{J}=5.9$ and $14.7 \mathrm{~Hz}, 1 \mathrm{H})$; $3.21(\mathrm{~s}, 3 \mathrm{H}) ; 3.50(\mathrm{~m}, 1 \mathrm{H}) ; 3.83(\mathrm{~s}, 3 \mathrm{H}) ; 3.89(\mathrm{dd}, \mathrm{J}=6.6$ and $8.4 \mathrm{~Hz}, 1 \mathrm{H})$; 4.54 (m, 2H); 4.64 (m, 1H); 4.92 (bs, 1H); 5.21 (d, J = 10.5 Hz, 1H); 5.26$5.34(\mathrm{~m}, 2 \mathrm{H}) ; 5.83(\mathrm{bs}, 1 \mathrm{H}) ; 5.84-5.96(\mathrm{~m}, 1 \mathrm{H}) ; 6.10(\mathrm{~d}, \mathrm{~J}=7.7 \mathrm{~Hz}, 1 \mathrm{H})$; $6.70(\mathrm{bs}, 1 \mathrm{H}) ; 6.90(\mathrm{t}, \mathrm{J}=7.8 \mathrm{~Hz}, 2 \mathrm{H}) ; 7.15(\mathrm{bs}, 2 \mathrm{H}) ; 7.16-7.22(\mathrm{~m}, 2 \mathrm{H})$; 7.23-7.35 (m, 4H); $7.50(\mathrm{t}, \mathrm{J}=7.3 \mathrm{~Hz}, 2 \mathrm{H}) ; 7.77(\mathrm{~d}, \mathrm{~J}=8.0 \mathrm{~Hz}, 1 \mathrm{H}) .{ }^{13} \mathrm{C}-$ NMR (100 MHz, $\left.\mathrm{CDCl}_{3}\right): \delta(\mathrm{ppm}) 11.4(\mathrm{q}) ; 15.3(\mathrm{q}) ; 24.7(\mathrm{t}) ; 27.6(\mathrm{t}) ; 28.0$ $(3 \mathrm{q}) ; 37.2(\mathrm{t}) ; 37.8(\mathrm{~d}) ; 52.3(\mathrm{q}) ; 53.3(\mathrm{q}$ and d); $59.2(\mathrm{~d}) ; 59.5(\mathrm{~d}) ; 65.8(\mathrm{t}) ;$ 73.4 (s); 81.9 (s); 82.1 (d); 109.2 (s); 110.9 (d); 117.7 (t); 119.9 (2d); 120.5 (d); 122.7 (d); 124.3 (d); 125.7 (d); 126.4 (2d); 126.7 (d); 128.5 (2d); 130.3 (s); 130.6 (s); 131.7 (d); 132.2 (d); 132.7 (d); 133.5 (s); 138.2 (s); 143.2 (s); 154.8 (s); 155.9 (s); 170.1 (s); $170.5(\mathrm{~s}) ; 170.6(\mathrm{~s}) . \mathrm{IR}(\mathrm{KBr}): v\left(\mathrm{~cm}^{-1}\right) 3367$, 2954, 1721, 1447, 1363, $1170 \mathrm{~cm}^{-1}$. HRMS (ESI) calculated for $\mathrm{C}_{45} \mathrm{H}_{54} \mathrm{~N}_{5} \mathrm{O}_{11} \mathrm{~S} m / z\left(\mathrm{M}+\mathrm{H}^{+}\right)$872.3541, found 872.3557.

Compound 1h: Purified by flash chromatography (hexane/EtOAc, 60:40). ${ }^{1} \mathrm{H}-\mathrm{NMR}\left(400 \mathrm{MHz}, \mathrm{CDCl}_{3}\right): \delta(\mathrm{ppm}) 2.66(\mathrm{dd}, J=9.5$ and $5.3 \mathrm{~Hz}, 1 \mathrm{H})$; $2.79(\mathrm{~d}, J=13.3 \mathrm{~Hz}, 1 \mathrm{H}) ; 3.03-3.14(\mathrm{~m}, 1 \mathrm{H}) ; 3.20(\mathrm{~s}, 3 \mathrm{H}) ; 3.42-3.54(\mathrm{~m}$, $1 \mathrm{H})$; 3.58-3.68 (m, 6H); 3.84 (s, 3H); 4.42-4.57 (m, 1H); $4.91(\mathrm{bs}, 1 \mathrm{H})$; $5.10(\mathrm{~d}, J=8.1 \mathrm{~Hz}, 1 \mathrm{H}) ; 5.73(\mathrm{~s}, 1 \mathrm{H}) ; 6.71(\mathrm{bs}, 1 \mathrm{H}) ; 6.87(\mathrm{t}, J=7.5 \mathrm{~Hz}$, $2 \mathrm{H})$; 7.12-7.35 (m, 5H); 7.43-7.58 (m, 3H); $7.80(\mathrm{bd}, J=7.8 \mathrm{~Hz}, 1 \mathrm{H}) .{ }^{13} \mathrm{C}-$ NMR (100 MHz, $\left.\mathrm{CDCl}_{3}\right): \delta(\mathrm{ppm}) 28.0(\mathrm{t}) ; 37.5(\mathrm{t}) ; 52.5(3 \mathrm{q}) ; 53.5(\mathrm{q})$; 54.8 (d); 59.4 (d); 73.6 (s); 82.1 (d); 109.4 (s); 111.2 (d); 119.8 (d); 120.2 (d); 120.7 (d); 123.0 (d); 124.3 (d); 124.5 (s); 125.8 (s); 126.6 (2d); 126.8 (d); 128.7 (2d); 130.5 (s); 132.1 (2d); 132.4 (d); 133.7 (s); 138.3 (s); 143.5 (s); $156.5(\mathrm{~s}) ; 170.6(\mathrm{~s}) ; 172.3(\mathrm{~s})$. IR (KBr): v $\left(\mathrm{cm}^{-1}\right)$ 3328, 2964, 1722 , $1676,1448,1368,1170$. HRMS (ESI) calculated for $\mathrm{C}_{34} \mathrm{H}_{35} \mathrm{~N}_{4} \mathrm{O}_{10} \mathrm{~S} m / z$ $\left(\mathrm{M}+\mathrm{H}^{+}\right)$691.2074, found 691.2079.

Compound 1i: Purified by flash chromatography $\left(\mathrm{MeCN} / \mathrm{H}_{2} \mathrm{O}\right.$, from 30:70 to 50:50). ${ }^{1} \mathrm{H}-\mathrm{NMR}\left(400 \mathrm{MHz}, \mathrm{CDCl}_{3}\right): \delta$ (ppm) $0.84(\mathrm{~m}, 6 \mathrm{H}) ; 1.07(\mathrm{~m}$, $1 \mathrm{H}) ; 1.18(\mathrm{~s}, 9 \mathrm{H}) ; 1.40(\mathrm{~s}, 10 \mathrm{H}) ; 1.80(\mathrm{~m}, 1 \mathrm{H}) ; 2.80(\mathrm{~m}, 2 \mathrm{H}) ; 3.10(\mathrm{dd}, J=$ 5.9 and $14.6 \mathrm{~Hz}, 1 \mathrm{H}) ; 3.50(\mathrm{dd}, J=10.1$ and $13.4,1 \mathrm{H}) ; 3.81(\mathrm{~s}, 3 \mathrm{H}) ; 3.85$ $(\mathrm{m}, 1 \mathrm{H}) ; 4.41(\mathrm{dd}, J=5.7$ and $13.2 \mathrm{~Hz}, 1 \mathrm{H}) ; 4.51(\mathrm{dd}, J=5.7$ and $13.2 \mathrm{~Hz}$, $1 \mathrm{H}) ; 4.70-4.87(\mathrm{~m}, 2 \mathrm{H}) ; 5.10(\mathrm{bs}, 1 \mathrm{H}) ; 5.20(\mathrm{~m}, 2 \mathrm{H}) ; 5.70(\mathrm{~m}, 1 \mathrm{H}) ; 5.78(\mathrm{bs}$, $1 \mathrm{H}) ; 6.20(\mathrm{~d}, J=7.0 \mathrm{~Hz}, 1 \mathrm{H}) 6.65(\mathrm{bs}, 1 \mathrm{H}) ; 6.85(\mathrm{t}, J=7.6,2 \mathrm{H}) ; 7.25(\mathrm{~m}$, $8 \mathrm{H}) ; 7.50(\mathrm{~m}, 2 \mathrm{H}) ; 7.80(\mathrm{~d}, J=7.6 \mathrm{~Hz}, 1 \mathrm{H}) .{ }^{13} \mathrm{C}-\mathrm{NMR}\left(100 \mathrm{MHz}, \mathrm{CDCl}_{3}\right)$ : $\delta(\mathrm{ppm}) 11.6(\mathrm{q}) ; 15.4(\mathrm{q}) ; 24.7(\mathrm{t}) ; 27.7(3 \mathrm{q}) ; 28.2(\mathrm{t}) ; 28.4(3 \mathrm{q}) ; 31.5(\mathrm{q}) ;$ $36.6(\mathrm{q}) ; 37.3(\mathrm{t}) ; 37.7(\mathrm{~d}) ; 53.2(\mathrm{~d}) ; 59.1(\mathrm{~d}) ; 60.0(\mathrm{~d}) ; 66.0(\mathrm{t}) ; 79.8(\mathrm{~s}) ;$ 82.1 (d); 82.3 (s); 109.0 (d); 111.1 (d); 118.8 (t); 119.6 (d); 120.1 (s); 120.6 (d); 122.8 (d); 124.4 (d); 126.0 (d); 126.5 (d); 126.6 (d); 128.6 (2d); 130.4 (d); 131.0 (s); 131.4 (d); 131.7 (d); 132.3 (d); 133.7 (s); 138.3 (s); 141.7 (s); $143.2(\mathrm{~s}) ; 145.0(\mathrm{~s}) ; 155.6(\mathrm{~s}) ; 162.7$ (s); 168.8 (s); 171.0 (s); 171.3 (s). IR $(\mathrm{KBr}): v\left(\mathrm{~cm}^{-1}\right)$ 3323, 2965, 2929, 1716, 1448, 1367, 1171. HRMS (ESI) calculated for $\mathrm{C}_{48} \mathrm{H}_{60} \mathrm{~N}_{5} \mathrm{O}_{11} \mathrm{~S} \mathrm{~m} / z\left(\mathrm{M}+\mathrm{H}^{+}\right)$914.4010, found 914.3986.

Compound 1j: Purified by flash chromatography (hexane/EtOAc 70:30) ${ }^{1} \mathrm{H}-\mathrm{NMR}$ (400 MHz, $\mathrm{CDCl}_{3}$ ): $\delta$ (ppm) 0.85-1.03 (m, 6H); 1.13 (dd, $J=3.2$ and $6.7 \mathrm{~Hz}, 2 \mathrm{H}) ; 1.54(\mathrm{~s}, 9 \mathrm{H}) ; 1.56(\mathrm{~s}, 9 \mathrm{H}) ; 2.52(\mathrm{bs}, 1 \mathrm{H}) ; 2.68-2.90(\mathrm{~m}$, $1 \mathrm{H})$; 2.97-3.15 (m, 1H); 3.54-3.66 (m, 2H); $3.77(\mathrm{~s}, 3 \mathrm{H})$; 3.94-4.07 (m, 1H); 4.49-4.73 (m, 3H); 5.08-5.31 (m, 3H); 5.75-5.90 (m, 1H); 6.50 (d, $J=7.7$ $\mathrm{Hz}, 1 \mathrm{H}) ; 6.65(\mathrm{~d}, J=11.2 \mathrm{~Hz}, 1 \mathrm{H}) ; 6.75(\mathrm{~d}, J=9.2 \mathrm{~Hz}, 1 \mathrm{H}) ; 6.87-7.18(\mathrm{~m}$, $5 \mathrm{H}) ; 7.33-7.41(\mathrm{~m}, 1 \mathrm{H}) ; 7.61(\mathrm{dd}, J=6.1$ and $7.4 \mathrm{~Hz}, 1 \mathrm{H}) ; 7.66-7.83(\mathrm{~m}$, $5 \mathrm{H}) ; 7.87-8.01$ (bs, $1 \mathrm{H}) .{ }^{13} \mathrm{C}-\mathrm{NMR}\left(100 \mathrm{MHz} \mathrm{CDCl}_{3}\right): \delta(\mathrm{ppm}) 10.7$ and $11.3(\mathrm{q}) ; 15.8$ and $16.8(\mathrm{q}) ; 24.3(\mathrm{t}) ; 25.8$ and $27.4(\mathrm{t}) ; 28.2(6 \mathrm{q}) ; 34.1$ and $34.4(\mathrm{~d}) ; 39.9(\mathrm{t}) ; 52.2$ and $52.3(\mathrm{~d}) ; 52.8(\mathrm{q}) ; 56.8$ and $58.2(\mathrm{~d}) ; 61.2(\mathrm{~d})$; $61.5(\mathrm{~s}) ; 66.3(\mathrm{t}) ; 78.4$ (d); 78.7 (s); $83.1(2 \mathrm{~s}) ; 109.7$ (s); 110.7 (d); 111.0 (d); 116.0 and $116.1(\mathrm{~d}) ; 118.8(\mathrm{t}) ; 119.4$ (d); 120.3 (d); 122.8 (d); 123.3 (d); $123.4(\mathrm{~d}) ; 123.5$ and $123.6(\mathrm{~s}) ; 124.5(2 \mathrm{~d}) ; 126.4(\mathrm{~s}) ; 129.9$ (s); 131.4 (d); $131.5(\mathrm{~d}) ; 131.7$ (s); 134.1 (2d); $134.3(\mathrm{~s}) ; 134.4(\mathrm{~s}) ; 151.2(\mathrm{~s}) ; 167.1$ (s); $167.3(\mathrm{~s}) ; 168.0(\mathrm{~s}) ; 168.1(\mathrm{~s}) ; 169.3(2 \mathrm{~s})$. IR (KBr): v $\left(\mathrm{cm}^{-1}\right)$ 3413, 2969, 1718, 1483, 1455, 1388, 1253, 1162, 1019, 739, 720. HRMS (ESI+) calculated for $\mathrm{C}_{46} \mathrm{H}_{49} \mathrm{~N}_{5} \mathrm{O}_{11} \mathrm{~m} / z(\mathrm{M}-t \mathrm{Bu}) 847.3429$, found 847.3658. 


\section{Acknowledgments}

This study was partially supported by CICYT (Grant CTQ2009-07758), the Generalitat de Catalunya (2009SGR 1024), the Institute for Research in Biomedicine, and the Barcelona Science Park.

[1] P. Ruiz-Sanchis, S.A. Savina, F. Albericio, M. Álvarez; Chem. Eur. J. 2011, 17, 1388-1408

[2] B. Witkop, Heterocycles 1998, 49, 9-27.

[3] a) J.S. Carlé, C. Christophersen, J. Am. Chem. Soc. 1979, 101, 4012 4013. b) J.S. Carlé, C. Christophersen, J. Org. Chem. 1980, 45 , 1586-1589. c) J.S. Carlé, C. Christophersen, J. Org. Chem. 1981, 46, 3440-3443. d) M.V. Laycock, J.L.C. Wright, J.A. Findlay, A.D Patil, Can. J. Chem. 1986, 64, 1312-1316. e) P.B. Holst, U. Anthoni, C. Christophersen, P.H. Nielsen, J. Nat. Prod. 1994, 57, 997-1000. f) S.J. Rochfort, S. Moore, C. Craft, N.H. Martin, R.M Van Wagoner, J.L.C. Wright, J. Nat. Prod. 2009, 72, 1773-1781.

[4] a) M. Kusano, G. Sotoma, H. Koshino, J. Uzawa, M. Chijimatsu, S Fujioka, T. Kawano, Y. Kimura, J. Chem. Soc., Perkin Trans. 1 1998, 2823-2826. b) Y. Kimura, A. Sawada, M. Kuramata, M. Kusano, S. Fujioka, T. Kawano, A. Shimada, J. Nat. Prod. 2005, 68, 237-239. c) L. Du, X. Yang, T. Zhu, F. Wang, X. Xiao, H. Park, Q. Gu, Chem. Pharm. Bull. 2009, 57, 873-876.

[5] a) S. Ohmomo, T. Sato, T. Utagawa, M. Abe, Agric. Biol. Chem 1975, 39, 1333-1334. b) S. Ohmomo, T. Utagawa, M. Abe, Agric. Biol. Chem. 1977, 41, 2097-2098. c) S. Ohmomo, K. Oguma, T Ohashi, M. Abe, Agric. Biol. Chem. 1978, 12, 2387-2389. d) L. Du, D. Li, T. Zhu, S. Cai, F. Wang, X. Xiao, Q. Gu, Tetrahedron 2009, 65, 1033-1039

[6] R.K. Duke, R.D. Allan, G.A.R. Johnston, K.N. Mewett, A.D. Mitrovic, C.C. Duke, T.W. Hambley, J. Nat. Prod. 1995, 58, 12001208.

[7] N.K. Hart, S.R. Johns, J.A. Lamberton, R.E. Summons, Aust. J. Chem., 1974, 27, 639-646

[8] a) V. Jannic, F. Gueritte, O. Laprevote, L. Serani, M.-T. Martin, T Sévenet, P. Potier, J. Nat. Prod. 1999, 62, 838-843. b) L. Verotta, T. Pilati, M. Tato, E. Elisabetsky, T.A. Amador, D.S. Nunes, J. Nat Prod. 1998, 61, 392-396. c) F. Guéritte-Voegelein, T. Sévenet, J Pusset, M.-T. Adeline, B. Gillet, J.-C. Beloeil, D. Guénard, P. Potier, R. Rasolonjanahary, C. Kordon, J. Nat. Prod. 1992, 55, 923-930. d) F. Libot, C. Miet, N. Kunesch, J.E. Poisson, J. Pusset T. Sévenet, J. Nat. Prod. 1987, 50, 468-473. e) K.P. Parry, G.F Smith, J. Chem. Soc. Perkin Trans. 1 1978, 1671-1682.

[9] H. Takayama, I. Mori, M. Kitajima, N. Aimi, N.H. Lajis, Org. Lett. 2004, 6, 2945-2948.

[10] a) S.A. Waksman, E. Bugie, J. Bacteriol., 1944, 48, 527-530. b) W.B. Geiger, J.E. Conn, S.A. Waksman, J. Bacteriol. 1944, 48 , 531-536. c) W.B. Geiger, Arch. Biochem. 1949, 21, 125-131. d) S. Safe, A. Taylor, J. Chem. Soc. Perkin Trans. 1 1972, 472-479. e) A.G. McInnes, A. Taylor, J.A. Walter, J. Am. Chem. Soc. 1976, 98 6741-6741. f) T. Kikuchi, S. Kadota, K. Nakamura, A. Nishi, T. Taga, T. Kaji, K. Osaki, K. Tubaki, Chem. Pharm. Bull. 1982, 30, 3846-3848. g) G.-Y. Li, B.-G. Li, T. Yang, J.-F. Yan, G.-Y. Liu, G.-L. Zhang, J. Nat. Prod. 2006, 69, 1374-1376.
[11] a) Y. Nakao, B.K.S. Yeung, Y.W. Yoshida, P.J. Scheurer, J. Am. Chem. Soc. 1995, 117, 8271-8272; b) B.K.S. Yeung, Y. Nakao, R.B. Kinnel, J.R. Carney, W.Y. Yoshida, P.J. Scheuer, M. KellyBorges, J. Org. Chem. 1996, 61, 7168-7173; c) Y. Nakao, J. Kuo, W.Y. Yoshida, M. Kelly, P.J. Scheuer, Org. Lett. 2003, 5, 13871390 .

[12] a) Y. Matsuda, M. Kitajima, H. Takayama, Org. Lett. 2008, 10, 125-128; b) T. Newhouse, P.S. Baran, J. Am. Chem. Soc. 2008 130, 10886-10887; c) N. Takahashi, T. Ito, Y. Matsuda, N. Kogure, M. Kitajima, H. Takayama, Chem. Commun. 2010, 46, 2501-2503 d) T. Newhouse, C.A. Lewis, K.J. Eastman, P.S. Baran, J. Am. Chem. Soc. 2010, 132, 7119-7137.

[13] T. Newhouse, C.A. Lewis, P.S. Baran, J. Am. Chem. Soc. 2009 $131,6360-6361$.

[14] K. Foo, T. Newhouse, I.Mori, H. Takayama, P.S. Baran, Angew. Chem. Int. Ed. 2011, 50, 2716-2719

[15] V.R. Espejo, J.D. Rainier, J. Am. Chem. Soc. 2008, 130, 1289412895

[16] V.R. Espejo, J.D. Rainier, Org. Lett. 2010 12, 2154-2157

[17] V.R. Espejo, J.D. Rainier, J. Am. Chem. Soc. 2010, 132, 8282-8284

[18] M. Taniguchi, T. Hino, Tetrahedron 1981, 37, 1487

[19] C. Silva-López, C. Pérez-Balado, P. Rodríguez-Granja, A.R. de Lera, Org. Lett., 2008, 10, 77-80

[20] Column: Waters XBridge $\mathrm{C}_{18}(75 \times 4.6 \mathrm{~mm}, 2.5 \mu \mathrm{m})$; eluent and gradient: $\mathrm{MeCN} / \mathrm{H}_{2} \mathrm{O}$ (from 30:70 to 100:0 in 8 minutes)

[21] Several reaction conditions were tested to remove the Moc group from methyl $N^{I}$-(methoxycarbonyl)- $N^{8}$-(phenylsulphonyl)HPI-2carboxylate (S6, see Supporting Information): the reagents comprised $\mathrm{LiOH}, \mathrm{LiSBn}, \mathrm{LiSCH}_{2} \mathrm{CH}_{2} \mathrm{OH}$ or TMSI; the solvents tested were THF, $\mathrm{H}_{2} \mathrm{O}, 1$,4-dioxane, $\mathrm{MeOH}$ and HMPA; and the temperatures, from rt to solvent reflux temperature. Several of these tests were performed on MW.

[22] a) M. Bruncko, D. Crich, R. Samy, J. Org. Chem. 1994, 59, 55435549; b) M. Kawahara, A. Nishida, M. Nakagawa, Org. Lett. 2000, $2,675-678$

[23] a) T.M. Kamenecka, S.J. Danishefsky Angew. Chem. Int. Ed. 1998 , 37, 2993-2995; b) T.M. Kamenecka, S.J. Danishefsky Angew. Chem. Int. Ed. 1998, 37, 2995-2998

[24] N.H. Martin, N.W. Allen III, K.D. Moore, L. Vo, J. Mol. Struct 1998, $454,161-166$

[25] The 3D model of Br-HPI endo- and exo-3g was calculated with Gaussian ${ }^{\circledR} 03 \mathrm{~W}$ V 6.0 (Gaussian Inc.) and built with ChemBio3D V 11.0 (CambridgeSoft).

[26] Base-solvent pairs such as NaH/THF, NaHMDS/THF, $\mathrm{K} t \mathrm{BuO} / \mathrm{MeCN}$ or proton sponge/THF did not provide better yields.

[27] V.R. Espejo, X.-B. Li, J.D. Rainier, J. Am. Chem. Soc. 2010, 132 8282-8284

Received: ((will be filled in by the editorial staff)) Published online: ((will be filled in by the editorial staff)) 
Entry for the Table of Contents ((Please choose one layout.))

\section{Layout 1:}

Trp-HPI

Several Tryptophanyl-

Hexahydropyrroloindoles (Trp-HPI) with four or five orthogonal protecting groups have been synthesized. This polyheterocyclic system is the scaffold of many natural products, recently isolated.

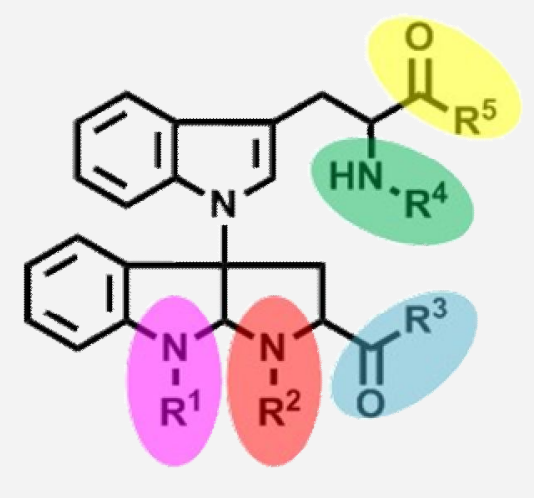

Pau Ruiz-Sanchis, Svetlana A. Savina, Gerardo A. Acosta, Fernando Albericio," and Mercedes Álvarez Page No. - Page No.

Orthogonal Protecting Groups in the Synthesis of TryptophanylHexahydropyrroloindoles

Keywords: Natural products / Heterocycles / Amino acids / Protecting groups 


\title{
Orthogonal Protecting Groups in the Synthesis of Tryptophanyl-Hexahydropyrroloindoles
}

\author{
Pau Ruiz-Sanchis, ${ }^{[a, b]}$ Svetlana A. Savina, ${ }^{[a, c]}$ Gerardo A. Acosta, ${ }^{[a, c]}$
}

Fernando Albericio, ${ }^{*[a, c, d]}$ and Mercedes Álvarez ${ }^{*[a, b, c]}$

[a]: Institute for Research in Biomedicine, Barcelona Science Park, Baldiri Reixac 10, 08028 Barcelona, Spain; [b]: Laboratory of Organic Chemistry, Faculty of Pharmacy, University of Barcelona, 08028 Barcelona, Spain. [c]: ClBER-BBN, Networking Center on Bioengineering, Biomaterials and Nanomedicine, Barcelona Science Park, Baldiri Reixac 10, 08028 Barcelona, Spain; [d]: Department of Organic Chemistry, University of Barcelona, 08028 Barcelona, Spain

albericio@irbbarcelona.org, mercedes.alvarez@irbbarcelona.org

\section{TABLE OF CONTENTS}

SI 2 General data

SI 2 Experimental section

SI 5 Compounds characterization

SI $12 \quad{ }^{1} \mathrm{H}$ - and ${ }^{13} \mathrm{C}$-NMR spectra 


\section{General data}

Reagents and solvents were purified according to Purification of Laboratory Chemicals (Armarego, W. and Chai, C.; Elsevier; 2003). Automatic flash chromatography was done in an Isco Combiflash medium pressure liquid chromatograph with Redisep silica gel columns $(47-60 \mu \mathrm{m}) .{ }^{1} \mathrm{H}$ - and ${ }^{13} \mathrm{C}-\mathrm{NMR}$ spectra were recorded on a Varian Mercury $400 \mathrm{MHz}$. Multiplicity of the carbons was assigned with DEPT and gHSQC experiments, although standard abbreviations according to off-resonance decoupling are used here: (s) singlet, (d) doublet, (t) triplet and (q) quartet. For ${ }^{1} \mathrm{H}-\mathrm{NMR}$, the following additional abbreviations are used: (m) multiplet, (bs) broad singlet and (bd) broad doublet. Spectra were referenced to the residual solvent peak of $\mathrm{CDCl}_{3}$. IR spectra were obtained on a Thermo Nicolet FT-IR spectrometer. HRMS was performed on an Acquity UPLC Binary Sol MGR (WatersCorporation) by the Mass Spectrometry Core Facility at the IRBB. Analytical HPLC was performed on a Waters Alliance 2695 separation module equipped with a Waters 996 PDA detector $(\lambda=254 \mathrm{~nm})$ and Waters XBridge $C_{18}$ column $(75 \times 4.6 \mathrm{~mm}, 2.5 \mu \mathrm{m})$, in runs of $8 \mathrm{~min}$. Microwave-assisted reactions were carried out in a CEM Discover microwave oven.

\section{Experimental section}

$N^{\alpha}$-Protection of L-Trp-OMe $\left(R^{2}=\right.$ Alloc, Boc, Cbz, Moc, Troc, $\boldsymbol{N}^{\alpha}$-Alloc-Ala)

A solution of L-Trp-OMe.HCl $(5.4 \mathrm{~g}, 21.2 \mathrm{mmol})$ and $\mathrm{Et}_{3} \mathrm{~N}(2.9 \mathrm{~mL}, 21.2 \mathrm{mmol})$ in dry $\mathrm{CH}_{2} \mathrm{Cl}_{2}(85 \mathrm{~mL})$ was added to a solution of either di-tert-butyldicarbonate $(5.5 \mathrm{~g}, 25.4 \mathrm{mmol})$ or an appropriate chloroformate $(1.5 \mathrm{eq})$ in $\mathrm{CH}_{2} \mathrm{Cl}_{2}$ $(3 \mathrm{~mL} / \mathrm{mmol})$. The reaction mixture was stirred for $2 \mathrm{~h}$ at $\mathrm{rt}$. The organic solution was washed with brine, and then dried over $\mathrm{Na}_{2} \mathrm{SO}_{4}$. The solvent was removed, and the crude was purified by flash chromatography (hexanes/EtOAc) to afford the desired product in 80 to $99 \%$ yield.

If $\mathrm{R}^{2}=N^{\alpha}$-Alloc-Ala. A solution of Alloc-Ala-OH dicyclohexylamine salt $(4.1 \mathrm{~g}, 11.7 \mathrm{mmol})$, EDC.HCl $(2.3 \mathrm{~g}, 11.9$ mmol) and $\mathrm{HOBt}(1.6 \mathrm{~g}, 11.9 \mathrm{mmol})$ in dry $\mathrm{CH}_{2} \mathrm{Cl}_{2}(20 \mathrm{~mL})$ was added to a solution of Trp-OMe $(2.9 \mathrm{~g}, 11.5 \mathrm{mmol})$ and DIEA (4.2 $\mathrm{mL}, 24.1 \mathrm{mmol})$ in dry $\mathrm{CH}_{2} \mathrm{Cl}_{2}(16 \mathrm{~mL})$. The mixture was stirred for $4 \mathrm{~h}$ at rt. The organic solution was washed with sat. $\mathrm{NH}_{4} \mathrm{Cl}, 10 \% \mathrm{NaHCO}_{3}$, water and brine, dried over $\mathrm{Na}_{2} \mathrm{SO}_{4}$, and then concentrated. The resulting crude was purified by flash chromatography $\left(\mathrm{CH}_{2} \mathrm{Cl}_{2} / \mathrm{MeOH}, 99: 1\right)$ to obtain the desired product in $58 \%$ yield.

\section{Syntheses of $N^{\alpha}$-protected-Trp-OtBu analogs $\left(2 \mathrm{f}, 2 \mathrm{~h}, 2 \mathrm{k}, 2 \mathrm{~m} ; \mathbf{R}^{1}=\mathrm{H}\right)$}

Hydrolysis of the methyl ester. $N^{\alpha}$-protected-Trp-methyl ester $(9.9 \mathrm{mmol})$ was dissolved in $10: 1 \mathrm{THF} / \mathrm{H}_{2} \mathrm{O}(168 \mathrm{~mL})$ and $2 \mathrm{M} \mathrm{LiOH}(15 \mathrm{~mL}, 30,0 \mathrm{mmol})$, and the solution was stirred at $\mathrm{rt}$ for $3 \mathrm{~h}$. The solution was then diluted with water and subsequently brought to $\mathrm{pH} 5$ by dropwise addition of $2 \mathrm{~N} \mathrm{HCl}$. The aqueous solution was saturated with $\mathrm{NaCl}$ and the phases were separated. The aqueous layer was extracted with THF. The combined organic layers were dried over $\mathrm{Na}_{2} \mathrm{SO}_{4}$ and concentrated in vacuo to give the carboxylic acid in quantitative yield.

Formation of the tert-butyl ester. A mixture of $N^{\alpha}$-protected-Trp $(8.4 \mathrm{mmol}), \mathrm{BnEt}_{3} \mathrm{NCl}(1.9 \mathrm{~g}, 8.4 \mathrm{mmol})$ and $\mathrm{K}_{2} \mathrm{CO}_{3}$ $(7.6 \mathrm{~g}, 54.7 \mathrm{mmol})$ in $\mathrm{MeCN}(25 \mathrm{~mL})$ was vigorously stirred for $5 \mathrm{~h} . t-\mathrm{BuBr}(9.9 \mathrm{~mL}, 88.4 \mathrm{mmol})$ was then added, and the solution was heated at $50{ }^{\circ} \mathrm{C}$ for $2 \mathrm{~h}$. The reaction mixture was treated with $\mathrm{MeCN}(13 \mathrm{~mL})$, and then stirred for $24 \mathrm{~h}$. The solvent was evaporated off and the resulting solid was dissolved in 2:1 $\mathrm{EtOAc} / \mathrm{H}_{2} \mathrm{O}$. The aqueous solution was extracted with EtOAc. The combined organic layers were washed with brine and dried over $\mathrm{Na}_{2} \mathrm{SO}_{4}$. The solvent was removed to give the tert-butyl $N^{\alpha}$-protected-Trp carboxylates $\mathbf{2 f}(84 \%), \mathbf{2 k}(84 \%), \mathbf{2} \mathbf{h}$ (55\%) or $\mathbf{2 m}(58 \%)$. 


\section{Syntheses of $N^{\alpha}, N^{\prime}$-protected-Trp-alkyl esters (2a-2n)}

Compounds $\mathbf{2} \mathbf{c}^{1}$ and $\mathbf{2 \mathbf { l } ^ { 2 }}$ have previously been reported.

$N^{\alpha}$-Protected-Trp alkyl ester (13.3 mmol), $\mathrm{Bu}_{4} \mathrm{NHSO}_{4}(0.4 \mathrm{~g}, 1.3 \mathrm{mmol})$ and crushed $\mathrm{NaOH}(1.6 \mathrm{~g}, 39.9 \mathrm{mmol})$ were mixed in $\mathrm{CH}_{2} \mathrm{Cl}_{2}(26 \mathrm{~mL})$. Di-tert-butyl dicarbonate $(4.3 \mathrm{~g}, 19.9 \mathrm{mmol})$ was then added slowly, and the resulting mixture was stirred for $15 \mathrm{~h}$ at rt. The solvent was evaporated off, the crude was dissolved in 1:1 sat. $\mathrm{NH}_{4} \mathrm{Cl} / \mathrm{EtOAc}$ and extracted with EtOAc. The organic layer was washed with $\mathrm{H}_{2} \mathrm{O}$ and dried over $\mathrm{Na}_{2} \mathrm{SO}_{4}$. The solvent was removed, and the crude was purified by flash chromatography (hexanes/EtOAc, 80:20 to 60:40) to give $\mathbf{2 a}(68 \%), \mathbf{2} \mathbf{d}(32 \%), \mathbf{2 e}(42 \%)$ or $\mathbf{2 n}(16 \%)$.

For Nosyl protection, either $N^{\alpha}$-Cbz-Trp-OtBu, $N^{\alpha}$-Troc-Trp-OMe or $N^{\alpha}$-Troc-Trp-OtBu $(2.5 \mathrm{mmol})$ was treated with 2-nitrobenzenesulfonyl chloride $(0.7 \mathrm{~g}, 3.1 \mathrm{mmol})$, which provided the corresponding 8-(2-nitrobenzenesulfonyl) analogs $\mathbf{2 f}(80 \%), \mathbf{2 g}(43 \%)$ or $\mathbf{2 h}(46 \%)$, respectively.

For $\mathrm{SO}_{2} \mathrm{Ph}$ protection, either $N^{\alpha}$-Boc-Trp-OMe, $N^{\alpha}$-Cbz-Trp-OMe, $N^{\alpha}$-Cbz-Trp-OtBu, or $N^{\alpha}$-Moc-Trp-OtBu (23.9 mmol) was treated with $\mathrm{PhSO}_{2} \mathrm{Cl}(3.7 \mathrm{~mL}, 28.7 \mathrm{mmol})$ in $\mathrm{CH}_{2} \mathrm{Cl}_{2}(120 \mathrm{~mL})$. The reaction mixture was stirred for $2 \mathrm{~h}$ to give $\mathbf{2} \mathbf{i}(87 \%), \mathbf{2} \mathbf{j}(92 \%), \mathbf{2 k}(80 \%)$ or $\mathbf{2} \mathbf{m}(93 \%)$, respectively.

\section{Synthesis of 3a-bromo-1,2,3,3a,8,8a-hexahydropyrrolo[2,3-b]indole-2-carboxylate (3)}

\section{Method A: three-step synthesis for endo-3g, endo-3l and endo-3m}

endo-(2S, 3aS, 8aS)-Methyl 3a-bromo-8-(2-nitrobenzenesulfonyl)-1-trichloroethylcarbonyl--1,2,3,3a,8,8ahexahydropyrrolo[2,3-b]indole-2-carboxylate (endo-3g)

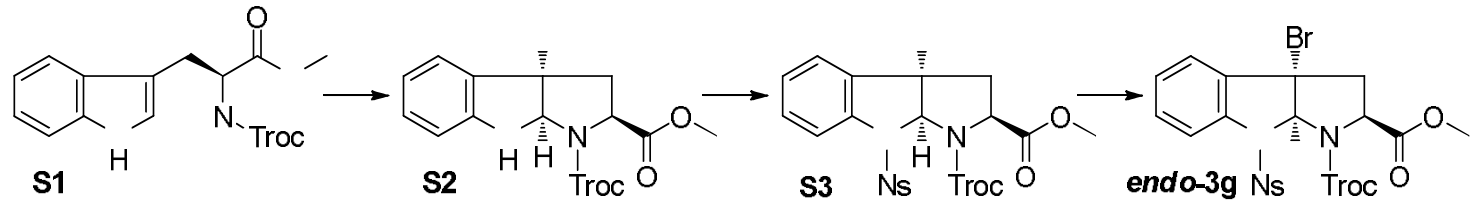

A solution of $N^{\alpha}$-Troc-Trp-OMe $(1.0 \mathrm{~g}, 2.5 \mathrm{mmol})$ and trifluoroacetic acid $(6.3 \mathrm{~mL})$ was stirred for 30 minutes at $\mathrm{rt}$, and then slowly poured into a mixture of $\mathrm{CH}_{2} \mathrm{Cl}_{2}(30.5 \mathrm{~mL})$ and $15 \% \mathrm{Na}_{2} \mathrm{CO}_{3}(76.2 \mathrm{~mL})$. The aqueous layer was extracted with $\mathrm{CH}_{2} \mathrm{Cl}_{2}$. The combined organic layers were dried over $\mathrm{Na}_{2} \mathrm{SO}_{4}$. The solvent was removed and the resulting residue was dried in vacuo to give $\mathbf{S} 2$ in quantitative yield.

2-Nitrobenzenesulfonyl chloride $(2.0 \mathrm{~g}, 9.2 \mathrm{mmol})$ was slowly added to a mixture of the previously $\mathbf{S} 2(0.9 \mathrm{~g}, 2.3$ $\mathrm{mmol}$ ) and pyridine $(3.0 \mathrm{~mL})$ cooled to $0 \stackrel{\circ}{\circ} \mathrm{C}$ and the reaction mixture was stirred at it for $2 \mathrm{~h}$. The organic solvent was removed in vacuo and the residue was dissolved in EtOAc. The organic solution was washed with $2 \mathrm{M} \mathrm{HCl}$, $15 \% \mathrm{Na}_{2} \mathrm{CO}_{3}$ and brine, dried over $\mathrm{Na}_{2} \mathrm{SO}_{4}$ and concentrated in vacuo. The resulting residue was purified by flash chromatography (hexanes/EtOAc, 3:1) to give S3 $(0.9 \mathrm{~g}, 65 \%)$.

AIBN $(0.1 \mathrm{~g}, 0.7 \mathrm{mmol})$ was added to a solution of $\mathbf{S} 3(0.9 \mathrm{~g}, 1.5 \mathrm{mmol})$ in dry $\mathrm{CCl}_{4}(28 \mathrm{~mL})$ under $\mathrm{N}_{2}$. The solution was heated to reflux, and then treated with NBS $(0.3 \mathrm{~g}, 1.5 \mathrm{mmol})$, the resulting mixture was stirred for $2 \mathrm{~h}$. The crude was then cooled to rt and the precipitate was filtered off. The organic layer was concentrated in vacuo and the resulting residue was purified by flash chromatography (hexanes/EtOAc, 80:20 to 60:40) to give endo-3g (0.1 $\mathrm{g}, 14 \%)$. The starting material was recovered.

endo-(2S, 3aS, 8aS)-Methyl 3a-bromo-1-methoxycarbonyl-8-benzenesulfonyl-1,2,3,3a,8,8a-hexahydropyrrolo[2,3b]indole-2-carboxylate (endo-3l).

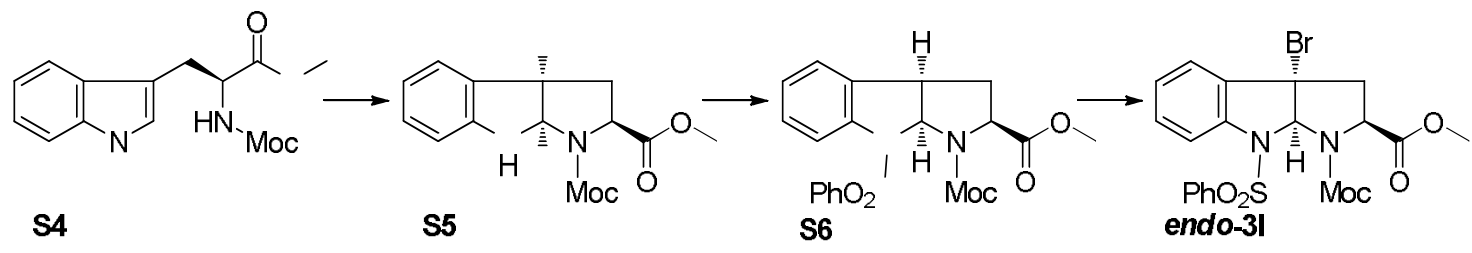

\footnotetext{
${ }^{1}$ S.P. Marsden, K.M. Depew, S.J. Danishefsky; J. Am. Chem. Soc.,1994, 116, 11143-11144.

${ }^{2}$ D. Crich, X. Huang; J. Org. Chem., 1999, 64, 7218-7223
} 
A solution of $N^{\alpha}$-Moc-Trp-OMe $(1.0 \mathrm{~g}, 3.6 \mathrm{mmol})$ and $85 \% \mathrm{H}_{3} \mathrm{PO}_{4}(11 \mathrm{~mL})$ was stirred under $\mathrm{N}_{2}$ at $\mathrm{rt}$ for $3 \mathrm{~h}$. The reaction mixture was then slowly poured into a mixture of $\mathrm{CH}_{2} \mathrm{Cl}_{2}(43 \mathrm{~mL})$ and $15 \% \mathrm{Na}_{2} \mathrm{CO}_{3}(109 \mathrm{~mL})$, and the resulting mixture was extracted with $\mathrm{CH}_{2} \mathrm{Cl}_{2}$. The combined organic layer was dried over $\mathrm{Na}_{2} \mathrm{SO}_{4}$. The solvent was removed and the residue was dried in vacuo to give $\mathbf{S} 5$ in quantitative yield.

Benzenesulfonyl chloride $(0.8 \mathrm{~mL}, 6.4 \mathrm{mmol})$ was slowly added to a mixture of S5 $(0.9 \mathrm{~g}, 3.2 \mathrm{mmol})$ and pyridine $(4 \mathrm{~mL})$ cooled to $0{ }^{\circ} \mathrm{C}$ and the reaction mixture was stirred at $\mathrm{rt}$ for $15 \mathrm{~h}$. The organic solvent was then removed in vacuo and the residue was dissolved in EtOAc. The organic solution was washed with $2 \mathrm{M} \mathrm{HCl}, 15 \% \mathrm{Na}_{2} \mathrm{CO}_{3}$ and brine, dried over $\mathrm{Na}_{2} \mathrm{SO}_{4}$ and concentrated in vacuo. The resulting residue was purified by flash chromatography (hexanes/EtOAc, 3:1) to give $\mathbf{S 6}(1.1 \mathrm{~g}, 80 \%)$.

endo-3l was obtained with $49 \%$ yield from $\mathbf{S} 6$ following the same reported method for endo-3g.

endo-(2S, 3aS, 8aS)-tert-Butyl 3a-bromo-1-methoxycarbonyl-8-benzenesulfonyl-1,2,3,3a,8,8a-

hexahydropyrrolo[2,3-b]indole-2-carboxylate (endo-3m).

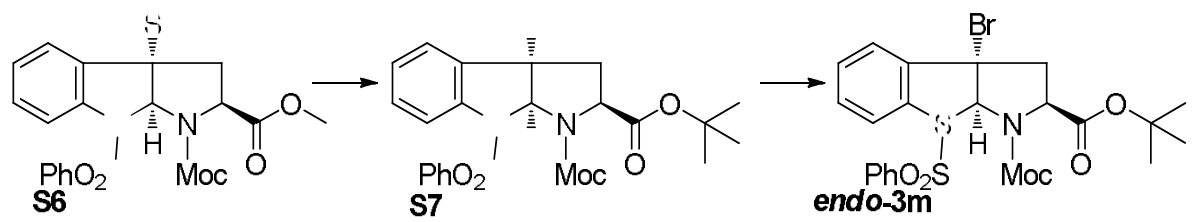

To a solution of $\mathbf{S 6}(5.0 \mathrm{~g}, 12.0 \mathrm{mmol})$ in $10: 1 \mathrm{THF} / \mathrm{H}_{2} \mathrm{O}(204 \mathrm{~mL})$ was added $2 \mathrm{M} \mathrm{LiOH}(12.0 \mathrm{~mL}, 24.0 \mathrm{mmol})$. The reaction mixture was stirred at reflux temperature for $2.5 \mathrm{~h}$. The solution was diluted with water and subsequently brought to $\mathrm{pH} 5$ by dropwise addition of $2 \mathrm{~N} \mathrm{HCl}$. The aqueous solution was saturated with $\mathrm{NaCl}$ and extracted with THF. The combined organic layers were dried over $\mathrm{Na}_{2} \mathrm{SO}_{4}$, and then concentrated in vacuo to give the carboxylic acid in quantitative yield.

Following the aforementioned method to generate a tert-butyl ester, $\mathbf{S 7}$ was obtained with $81 \%$ yield.

endo-3m was obtained in $46 \%$ yield from $\mathbf{S} 7$ following the same method reported for endo-3g.

\section{Method B: One-pot bromocyclization for exo-3a, exo-(3c-3n)}

To a solution of PPTS $(1.9 \mathrm{~g}, 7.4 \mathrm{mmol})$ and NBS $(1.3 \mathrm{~g}, 7.4 \mathrm{mmol})$ was added $N^{\alpha}$ - $N$-protected-Trp alkyl ester $(7.4$ mmol) in anhydrous $\mathrm{CH}_{2} \mathrm{Cl}_{2}(67 \mathrm{~mL})$ under $\mathrm{N}_{2}$. The reaction mixture was stirred at it for $4 \mathrm{~h}$. The crude mixture was washed with $15 \% \mathrm{NaHCO}_{3}, 10 \% \mathrm{Na}_{2} \mathrm{~S}_{2} \mathrm{O}_{4}$ and brine, and then dried over $\mathrm{Na}_{2} \mathrm{SO}_{4}$. The solvent was evaporated off, and the crude was purified by flash chromatography (see the following section for yields and solvent systems).

\section{Synthesis of exo-3b}

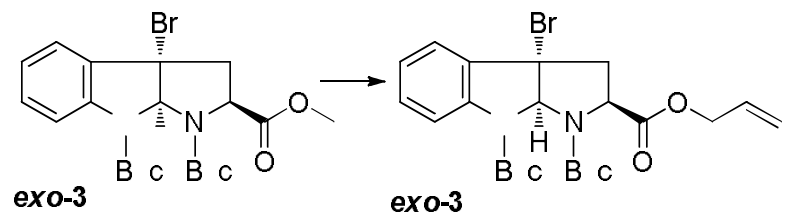

$2 \mathrm{M} \mathrm{LiOH}(1.5 \mathrm{~mL}, 3.0 \mathrm{mmol})$ was added to a solution of exo-3c $(0.5 \mathrm{~g}, 1.0 \mathrm{mmol})$ in $10: 1 \mathrm{THF} / \mathrm{H}_{2} \mathrm{O}(17 \mathrm{~mL})$. The solution was stirred at reflux for $5 \mathrm{~h}$. The solution was then diluted with water and subsequently brought to $\mathrm{pH} 5 \mathrm{by}$ dropwise addition of $2 \mathrm{~N} \mathrm{HCl}$. The aqueous layer was saturated with $\mathrm{NaCl}$ and extracted with THF. The combined organic layers were dried over $\mathrm{Na}_{2} \mathrm{SO}_{4}$ and concentrated in vacuo to give the carboxylic acid in quantitative yield. To a solution of the aforementioned acid $(0.7 \mathrm{~g}, 1.5 \mathrm{mmol})$ in $\mathrm{MeOH}(10 \mathrm{~mL})$ was added $\mathrm{Cs}_{2} \mathrm{CO}_{3}(0.5 \mathrm{~g}, 1.6 \mathrm{mmol})$, and the solution was stirred for $30 \mathrm{~min}$ at rt. The reaction mixture was then concentrated, treated with dry DMF (5 $\mathrm{mL})$ and AllylBr $(0.25 \mathrm{~mL}, 3.0 \mathrm{mmol})$, and finally, stirred at $\mathrm{rt}$ for $4 \mathrm{~h}$. The organic solution was diluted with EtOAc and washed with $5 \% \mathrm{NaHCO}_{3}$ and brine. The organic phase was dried over $\mathrm{MgSO}_{4}$, and then concentrated in vacuo. The resulting residue was purified by flash chromatography (hexanes/EtOAc, 80:20 to 70:30) to give exo3b $(0.2 \mathrm{~g}, 30 \%)$.

\section{Synthesis of exo-3o and exo-3p}

exo-3a or exo-3c were hydrolyzed following the aforementioned method for hydrolysis of a methyl ester of exo-3c. A solution of DIEA $(0.63 \mathrm{~mL}, 3.6 \mathrm{mmol})$ and either lle-OMOM $(0.6 \mathrm{~g}, 3.6 \mathrm{mmol}$, for exo-3o) or lle-OAllyl $(0.6 \mathrm{~g}, 3.6$ $\mathrm{mmol}$, for exo-3p) in $\mathrm{CH}_{2} \mathrm{Cl}_{2}(14 \mathrm{~mL})$ was added to a solution of the acid obtained $(3.0 \mathrm{mmol})$ from either exo-3a 
or exo-3c, respectively, in $\mathrm{CH}_{2} \mathrm{Cl}_{2}(23 \mathrm{~mL})$. The mixture was stirred at $-10{ }^{\circ} \mathrm{C}$ for $10 \mathrm{~min}$, and then treated with $\operatorname{HBTU}(1.1 \mathrm{~g}, 3.0 \mathrm{mmol})$, and the resulting mixture was stirred at $\mathrm{rt}$ for $21 \mathrm{~h}$. The mixture was washed with $5 \%$ $\mathrm{NaHCO}_{3}$, sat. $\mathrm{NH}_{4} \mathrm{Cl}$ and brine, dried over $\mathrm{Na}_{2} \mathrm{SO}_{4}$, and concentrated in vacuo. The resulting crude was purified by flash chromatography (hexanes/EtOAc, $70: 30$ ) to afford exo-3o (47\%) or exo-3p (41\%).

\section{Compounds Characterization:}

endo-3I, ${ }^{3}$ has been reported previously.

exo-Methyl 1-allyloxycarbonyl-3a-bromo-8-(tert-butyloxycarbonyl)-1,2,3,3a,8,8a-hexahydropyrrolo[2,3b]indole-2-carboxylate (exo-3a).<smiles>COC(=O)C1CCC1(Br)c1ccccc1N(C)C</smiles>

Purified by flash chromatography (hexanes:EtOAc 30:70) 83\% yield; exo.

${ }^{1} \mathrm{H}-\mathrm{RMN}\left(400 \mathrm{MHz}, \mathrm{CDCl}_{3}\right): \delta(\mathrm{ppm}) 1.59(\mathrm{~s}, 9 \mathrm{H}) ; 2.86(\mathrm{dd}, J=10.3$ and $12.7 \mathrm{~Hz}, 1 \mathrm{H}) ; 3.26$ (dd, $J=6.5$ and 12.7 $\mathrm{Hz}, 1 \mathrm{H}) ; 3.74(\mathrm{~s}, 3 \mathrm{H}) ; 3.97(\mathrm{dd}, J=6.5$ and $10.3 \mathrm{~Hz}, 1 \mathrm{H}) ; 4.43-4.70(\mathrm{~m}, 2 \mathrm{H}) ; 5.14-5.29(\mathrm{~m}, 2 \mathrm{H}) ; 5.75-5.94(\mathrm{~m}, 1 \mathrm{H})$; $6.40(\mathrm{~s}, 1 \mathrm{H}) ; 7.13(\mathrm{dd}, J=7.5$ and $7.6 \mathrm{~Hz}, 1 \mathrm{H}) ; 7.33$ (dd, $J=7.5$ and $8.1 \mathrm{~Hz}, 1 \mathrm{H}) ; 7.37(\mathrm{~d}, J=7.6 \mathrm{~Hz}, 1 \mathrm{H}) ; 7.60$ (bs, $1 \mathrm{H})$.

${ }^{13} \mathrm{C}-\mathrm{RMN}\left(100 \mathrm{MHz}, \mathrm{CDCl}_{3}\right)$ : $\delta(\mathrm{ppm}) 28.2(3 \mathrm{q}) ; 41.8$ (t); 52.6 (q); 59.5 (d); 59.7 (s); 66.4 (t); 82.3 (s); 83.8 (d); 117.7 (t); 118.4 (d); 123.2 (d); 124.5 (d); 130.8 (d); 132.2 (d); 132.5 (s); 141.3 (s); 152.0 (s); 171.1 (s).

IR (KBr): v $\left(\mathrm{cm}^{-1}\right)$ 2978, 1717, 1479, 1405, 1334, 1156, 1017, 754.

HRMS (ESI+) calculated for $\mathrm{C}_{21} \mathrm{H}_{25} \mathrm{BrN}_{2} \mathrm{O}_{6} \mathrm{Na} \mathrm{m} / z\left(\mathrm{M}+\mathrm{Na}^{+}\right) 503.0794$, found 503.0800 .

exo-Allyl 3a-bromo-1,8-di-tert-butyloxycarbonyl-1,2,3,3a,8,8a-hexahydropyrrolo[2,3-b]indole-2-carboxylate (3b).<smiles></smiles>

${ }^{1} \mathrm{H}-\mathrm{RMN}\left(400 \mathrm{MHz}, \mathrm{CDCl}_{3}\right): \delta(\mathrm{ppm}) 1.40(\mathrm{~s}, 9 \mathrm{H}) ; 1.59(\mathrm{~s}, 9 \mathrm{H}) ; 2.82(\mathrm{dd}, \mathrm{J}=10.3$ and $12.6 \mathrm{~Hz}, 1 \mathrm{H}) ; 3.23(\mathrm{dd}, \mathrm{J}=$ 6.4 and $12.6 \mathrm{~Hz}, 1 \mathrm{H})$; $3.91(\mathrm{dd}, \mathrm{J}=6.4$ and $10.3 \mathrm{~Hz}, 1 \mathrm{H})$; 4.56-4.70 $(\mathrm{m}, 2 \mathrm{H})$; 5.23-5.37 (m, 2H); 5.85-5.96 $(\mathrm{m}, 1 \mathrm{H})$; $6.39(\mathrm{~s}, 1 \mathrm{H}) ; 7.12(\mathrm{t}, \mathrm{J}=7.6 \mathrm{~Hz}, 1 \mathrm{H}) ; 7.32(\mathrm{dd}, \mathrm{J}=7.6$ and $8.0 \mathrm{~Hz}, 1 \mathrm{H}) ; 7.36(\mathrm{~d}, 7.6 \mathrm{~Hz}, 1 \mathrm{H}) ; 7.54(\mathrm{bs}, 1 \mathrm{H})$.

${ }^{13} \mathrm{C}-\mathrm{RMN}\left(100 \mathrm{MHz}, \mathrm{CDCl}_{3}\right): \delta(\mathrm{ppm}) 28.2(6 \mathrm{q}) ; 41.9(\mathrm{t}) ; 59.5(\mathrm{~d}) ; 59.7(\mathrm{~s}) ; 66.0(\mathrm{t}) ; 82.3(2 \mathrm{~s}) ; 83.8(\mathrm{~d}) ; 119.0(\mathrm{t}) ;$ 123.2 (2d); 124.4 (d); 130.6 (d); 131.5 (d); 133.1 (s); 141.5 (s); 152.2 (s); 171.0 (s).

IR (KBr): v $\left(\mathrm{cm}^{-1}\right)$ 2979, 1722, 1604, 1479, 1395, 1333, 1256, 1161, 1017, 851, 752.

HRMS (ESI+) calculated for $\mathrm{C}_{48} \mathrm{H}_{62} \mathrm{Br}_{2} \mathrm{~N}_{4} \mathrm{O}_{12} \mathrm{Na} \mathrm{m} / z\left(2 \mathrm{M}+\mathrm{Na}^{+}\right)$1067.2629, found 1067.2601.

exo-Methyl 3a-bromo-1,8-di(tert-butyloxycarbonyl)-1,2,3,3a,8,8a-hexahydropyrrolo[2,3-b]indole-2carboxylate (3c)<smiles>[B]C1([B])C(C(=O)OC)CC1(Br)c1ccccc1P</smiles>

Purified by flash chromatography (hexanes:EtOAc 80:20); 86\% yield; endo:exo 4:96.

${ }^{1} \mathrm{H}-\mathrm{RMN}\left(400 \mathrm{MHz}, \mathrm{CDCl}_{3}\right.$ ): $\delta(\mathrm{ppm}) 1.40(\mathrm{~s}, 9 \mathrm{H}) ; 1.59$ (s, 9H); $2.82(\mathrm{dd}, \mathrm{J}=10.3$ and $12.6 \mathrm{~Hz}, 1 \mathrm{H}) ; 3.20$ (dd, $\mathrm{J}=$ 6.3 and $12.6 \mathrm{~Hz}, 1 \mathrm{H}) ; 3.74(\mathrm{~s}, 3 \mathrm{H}) ; 3.89(\mathrm{dd}, \mathrm{J}=6.3$ and $10.3 \mathrm{~Hz}, 1 \mathrm{H}) ; 6.39(\mathrm{~s}, 1 \mathrm{H}) ; 7.12(\mathrm{dd}, \mathrm{J}=7.5 \mathrm{and} 7.6 \mathrm{~Hz}$, $1 \mathrm{H}) ; 7.32(\mathrm{dd}, \mathrm{J}=7.5$ and $8.1 \mathrm{~Hz}, 1 \mathrm{H}) ; 7.36(\mathrm{~d}, \mathrm{~J}=7.6 \mathrm{~Hz}, 1 \mathrm{H}) ; 7.56(\mathrm{bs}, 1 \mathrm{H})$.

\footnotetext{
${ }^{3}$ M. Bruncko, D. Crich, R. Samy, J. Org. Chem. 1994, 59, 5543-5549.
} 
${ }^{13} \mathrm{C}-\mathrm{RMN}\left(100 \mathrm{MHz}, \mathrm{CDCl}_{3}\right): \delta(\mathrm{ppm}) 28.5(3 \mathrm{q}) ; 28.6(3 \mathrm{q}) ; 42.2(\mathrm{t}) ; 52.7$ (q); $59.8(\mathrm{~d}) ; 60.1$ (s); $81.8(\mathrm{~s}) ; 82.6$ (s); 84.1 (d); 119.2 (d); 123.5 (d); 124.7 (d); 130.9 (d); 140.7 (s); 141.9 (s); 152.5 (s); 163.6 (s); 171.8 (s).

IR (KBr): v $\left(\mathrm{cm}^{-1}\right)$ 2979, 1720, 1394, 1333, 1161, 733.

HRMS (ESI+) calculated for $\mathrm{C}_{22} \mathrm{H}_{30} \mathrm{BrN}_{2} \mathrm{O}_{6} \mathrm{~S} \mathrm{~m} / z\left(\mathrm{M}+\mathrm{H}^{+}\right)$497.1282, found 497.1276.

exo-Methyl 1-benzyloxycarbonyl-3a-bromo-8-tert-butyloxycarbonyl-1,2,3,3a,8,8a-hexahydropyrrolo[2,3-

b]indole-2-carboxylate (3d)<smiles>COC(=O)C1CCC1(Br)c1ccccc1N(C)c1ccccc1</smiles>

Purified by flash chromatography (hexanes:EtOAc from 90:10 to $70: 30$ ); $78 \%$ yield; exo.

${ }^{1} \mathrm{H}-\mathrm{RMN}\left(400 \mathrm{MHz}, \mathrm{CDCl}_{3}\right.$ ): $\delta$ (ppm) 1.54 (bs, 9H); 2.85 (dd, $J=10.2$ and $12.8 \mathrm{~Hz}, 1 \mathrm{H}$ ); 3.26 (dd, $J=6.6$ and 12.8 $\mathrm{Hz}, 1 \mathrm{H}$ ); 3.65 (bs, 3H); 3.98 (dd, $J=6.6$ and $10.2 \mathrm{~Hz}, 1 \mathrm{H}$ ); 5.20 (bs, 2H); 6.43 (s, 1H); 7.13 (t, $J=7.5 \mathrm{~Hz}, 1 \mathrm{H}$ ); 7.25-7.38 (m, 7H); $7.61(\mathrm{~m}, 1 \mathrm{H})$.

${ }^{13} \mathrm{C}-\mathrm{RMN}\left(100 \mathrm{MHz}, \mathrm{CDCl}_{3}\right): \delta(\mathrm{ppm}) 28.1(3 \mathrm{q}) ; 41.8(\mathrm{t}) ; 52.4$ (q); 59.5 (d); 59.7 (s); 67.4 (t); 82.4 (s); 83.9 (d); 118.3 (d); 123.2 (d); 124.6 (d); 128.0 (s); 128.4 (4d); 130.8 (2d); 132.5 (s); 141.3 (s); 152.0 (s); 171.1 (s).

IR (KBr): v $\left(\mathrm{cm}^{-1}\right)$ 2978, 1717, 1478, 1410, 1335, 1157, 1018, 855, 753.

HRMS (ESI+) calculated for $\mathrm{C}_{25} \mathrm{H}_{27} \mathrm{BrN}_{2} \mathrm{O}_{6} \mathrm{Na} \mathrm{m} / z\left(\mathrm{M}+\mathrm{Na}^{+}\right)$553.0950, found 553.0950.

exo-Methyl 1-trichloroethoxycarbonyl-3a-bromo-8-(tert-butyloxycarbonyl)-1,2,3,3a,8,8ahexahydropyrrolo[2,3-b]indole-2-carboxylate (3e).

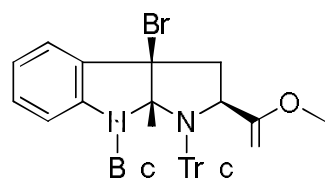

Purified by flash chromatography (hexanes:EtOAc 30:70) 77\% yield; endo:exo (11:89).

${ }^{1} \mathrm{H}-\mathrm{RMN}\left(400 \mathrm{MHz}, \mathrm{CDCl}_{3}\right.$ ): $\delta(\mathrm{ppm}) 1.61(\mathrm{~s}, 9 \mathrm{H}) ; 2.88(\mathrm{dd}, J=10.0$ and $13.0 \mathrm{~Hz}, 1 \mathrm{H}) ; 3.33$ (dd, $J=6.5$ and 13.0 $\mathrm{Hz}, 1 \mathrm{H}) ; 3.76(\mathrm{~s}, 3 \mathrm{H}) ; 4.05-4.13(\mathrm{~m}, 1 \mathrm{H}) ; 4.75$ and $4.78(2 \mathrm{~s}, 2 \mathrm{H}) ; 6.45(\mathrm{bs}, 1 \mathrm{H}) ; 7.14(\mathrm{t}, J=7.6 \mathrm{~Hz}, 1 \mathrm{H}) ; 7.34$ (dd, J $=7.6$ and $7.9,1 \mathrm{H}) ; 7.38(\mathrm{~d}, J=7.6 \mathrm{~Hz}, 1 \mathrm{H}) ; 7.68(\mathrm{bs}, 1 \mathrm{H})$.

${ }^{13} \mathrm{C}-\mathrm{RMN}\left(100 \mathrm{MHz}, \mathrm{CDCl}_{3}\right)$ : $\delta(\mathrm{ppm}) 29.6$ (q); 43.5 (t); 53.7 (s); 54.2 (q); 60.7 (d); 76.5 (t); 84.0 (s); 85.7 (d); 96.1 (s); 119.2 (d); 124.6 (d); 125.2 (s); 125.8 (d); 132.3 (d); 142.6 (s); 153.3 (2s); 172.2 (s).

IR $(\mathrm{KBr})$ : v $\left(\mathrm{cm}^{-1}\right)$ 2979, 1719, 1479, 1401, 1156, 1063, 754, 577.

HRMS (ESI+) calculated for $\mathrm{C}_{20} \mathrm{H}_{23} \mathrm{BrCl}_{3} \mathrm{~N}_{2} \mathrm{O}_{6} \mathrm{~m} / 2\left(\mathrm{M}+\mathrm{H}^{+}\right) 570.9805$, found 570.9791 .

exo-tert-Butyl 1-benzyloxycarbonyl-3a-bromo-8-(2-nitrobenzenesulfonyl)-1,2,3,3a,8,8ahexahydropyrrolo[2,3-b]indole-2-carboxylate (3f).<smiles>CC(C)(C)OC(=O)C1CC(Br)(c2ccccc2N)C1(N)N</smiles>

Purified by flash chromatography (hexanes:EtOAc 80:20) 80\% yield; endo:exo (7:93).

${ }^{1} \mathrm{H}-\mathrm{RMN}\left(400 \mathrm{MHz}, \mathrm{CDCl}_{3}\right): \delta(\mathrm{ppm}) 1.34(\mathrm{~s}, 9 \mathrm{H}) ; 2.80(\mathrm{dd}, J=10.5$ and $12.2 \mathrm{~Hz}, 1 \mathrm{H}) ; 3.10-3.19(\mathrm{~m}, 1 \mathrm{H}) ; 3.79$ (bs, $1 \mathrm{H}) ; 4.90$ and $4.93(2 \mathrm{~s}, 1 \mathrm{H}) ; 5.20(\mathrm{bs}, 1 \mathrm{H}) ; 6.54(\mathrm{~s}, 1 \mathrm{H}) ; 7.25-7.47(\mathrm{~m}, 10 \mathrm{H}) ; 7.55-7.65(\mathrm{~m}, 3 \mathrm{H})$.

${ }^{13} \mathrm{C}-\mathrm{RMN}\left(100 \mathrm{MHz}, \mathrm{CDCl}_{3}\right): \delta(\mathrm{ppm}) 27.9(3 \mathrm{q}) ; 43.3(\mathrm{t}) ; 59.2$ (s); 60.3 (d); 67.5 (t); 82.4 (d); 85.4 (s); 119.3 (d); 123.9 (2d); 124.2 (d); 127.0 (s); 128.1 (2d); 128.3 (2d); 128.4 (d); 129.9 (s); 131.1 (d); 131.4 (2d); 134.1 (d); 134.4 (s); $139.8(\mathrm{~s}) ; 148.6(\mathrm{~s}) ; 168.7(\mathrm{~s}) ; 171.1(\mathrm{~s})$.

IR $(\mathrm{KBr}): \mathrm{v}\left(\mathrm{cm}^{-1}\right)$ 2978, 1719, 1546, 1463, 1370, 1217, 1152, 1063, 594.

HRMS (ESI+) calculated for $\mathrm{C}_{29} \mathrm{H}_{29} \mathrm{BrN}_{3} \mathrm{O}_{8} \mathrm{~S} \mathrm{~m} / z\left(\mathrm{M}+\mathrm{H}^{+}\right) 658.0859$, found 658.0856. 
endo-Methyl 3a-bromo-8-(2-nitrobenzenesulfonyl)-1-trichloroethoxycarbonyl-1,2,3,3a,8,8ahexahydropyrrolo[2,3-b]indole-2-carboxilate $(3 \mathrm{~g})$

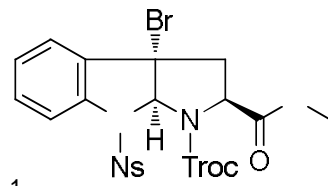

${ }^{1} \mathrm{H}-\mathrm{RMN}\left(400 \mathrm{MHz}, \mathrm{CDCl}_{3}\right): \delta(\mathrm{ppm}) 3.08-3.19(\mathrm{~m}, 1 \mathrm{H}) ; 3.21(\mathrm{~s}, 3 \mathrm{H}) ; 3.35(\mathrm{~d}, J=13.2 \mathrm{~Hz}, 1 \mathrm{H}) ; 4.50(\mathrm{~m}, 2 \mathrm{H}) ; 4.67$ $(\mathrm{d}, J=8.6 \mathrm{~Hz}, 1 \mathrm{H}) ; 6.59(\mathrm{~s}, 1 \mathrm{H}) ; 7.21(\mathrm{t}, J=7.2 \mathrm{~Hz}, 1 \mathrm{H}) ; 7.33-7.50(\mathrm{~m}, 3 \mathrm{H}) ; 7.57$ (t, $J=7.6 \mathrm{~Hz}, 1 \mathrm{H}) ; 7.69$ (t, $J=7.6$ $\mathrm{Hz}, 1 \mathrm{H}) ; 7.79(\mathrm{bd}, J=7.2 \mathrm{~Hz}, 1 \mathrm{H}) ; 8.03(\mathrm{bs}, 1 \mathrm{H})$.

${ }^{13} \mathrm{C}-\mathrm{RMN}\left(100 \mathrm{MHz}, \mathrm{CDCl}_{3}\right): \delta(\mathrm{ppm}) 44.5(\mathrm{t}) ; 52.5$ (q); 58.8 (s); 59.6 (d); 75.0 (t); 86.5 (d); $94.6(\mathrm{~s}) ; 117.9$ (d); 121.0 (s); 124.4 (d); 124.8 (d); 125.9 (d); 129.6 (d); 131.4 (d); 132.1 (d); 132.6 (s); 133.6 (d); 134.0 (s); 141.3 (s); $148.0(\mathrm{~s}) ; 169.4(\mathrm{~s}) ; 175.6(\mathrm{~s})$.

IR (KBr): v $\left(\mathrm{cm}^{-1}\right)$ 2994, 1736, 1545, 1463, 1376, 1228, 1175, 1048, 852, 767, 583.

HRMS (ESI+) calculated for $\mathrm{C}_{21} \mathrm{H}_{18} \mathrm{BrCl}_{3} \mathrm{~N}_{3} \mathrm{O}_{8} \mathrm{~S} \mathrm{~m} / z\left(\mathrm{M}+\mathrm{H}^{+}\right) 655.9064$, found 655.9041 .

exo-Methyl 3a-Bromo-8-(2-nitrobenzenesulfonyl)-1-trichloroethoxycarbonyl-1,2,3,3a,8,8ahexahydropyrrolo[2,3-b]indole-2-carboxilate $(3 \mathrm{~g})$

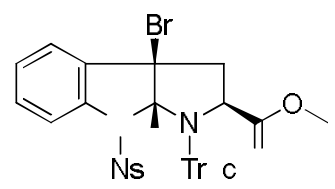

Purified by flash chromatography (DCM); $57 \%$ yield; endo:exo (8:92).

${ }^{1} \mathrm{H}-\mathrm{RMN}\left(400 \mathrm{MHz}, \mathrm{CDCl}_{3}\right.$ ): $\delta(\mathrm{ppm}) 2.90$ (dd, $J=10.1$ and $13.0 \mathrm{~Hz}, 1 \mathrm{H}$ ); 3.20 (dd, $J=6.2$ and $13.0 \mathrm{~Hz}, 1 \mathrm{H}$ ); 3.74 (s, 3H); 3.93-4.02 (m, 1H); 4.55 and $4.58(2 \mathrm{~s}, 2 \mathrm{H}) ; 6.60(\mathrm{~s}, 1 \mathrm{H}) ; 7.30(\mathrm{dd}, J=7.4$ and $7.6 \mathrm{~Hz}, 1 \mathrm{H}) ; 7.38(\mathrm{~d}, J=7.9$ $\mathrm{Hz}, 1 \mathrm{H}) ; 7.43(\mathrm{dd}, J=7.4$ and $7.9 \mathrm{~Hz}, 1 \mathrm{H}) ; 7.48(\mathrm{~d}, J=7.6 \mathrm{~Hz}, 1 \mathrm{H}) ; 7.57(\mathrm{~d}, J=7.3 \mathrm{~Hz}, 1 \mathrm{H}) ; 7.64(\mathrm{dd}, J=7.3$ and $8.0 \mathrm{~Hz}, 2 \mathrm{H}) ; 7.73-7.83(\mathrm{~m}, 1 \mathrm{H})$.

${ }^{13} \mathrm{C}-\mathrm{RMN}\left(100 \mathrm{MHz}, \mathrm{CDCl}_{3}\right): \delta(\mathrm{ppm}) 43.7$ (t); 52.8 (q); 58.7 (s); 59.5 (d); 75.1 (t); 86.1 (d); 94.8 (s); 119.6 (d); 123.9 (d); 124.3 (d); 127.2 (d); 130.3 (d); 131.3 (d); 131.4 (d); 132.0 (s); 134.1 (s); 134.3 (d); 139.9 (s); 148.9 (s); $160.1(\mathrm{~s}) ; 169.7$ (s).

IR $(\mathrm{KBr}): \mathrm{v}\left(\mathrm{cm}^{-1}\right)$ 2955, 1750, 1545, 1464, 1373, 1208, 1177, 1061, 774, 595.

HRMS (ESI+) calculated for $\mathrm{C}_{21} \mathrm{H}_{18} \mathrm{BrCl}_{3} \mathrm{~N}_{3} \mathrm{O}_{8} \mathrm{~S} \mathrm{~m} / z\left(\mathrm{M}+\mathrm{H}^{+}\right) 655.9064$, found 655.9045 .

exo-tert-Butyl 3a-bromo-8-(2-nitrobenzenesulfonyl)-1-trichloroethoxycarbonyl-1,2,3,3a,8,8ahexahydropyrrolo[2,3-b]indole-2-carboxylate (3h).

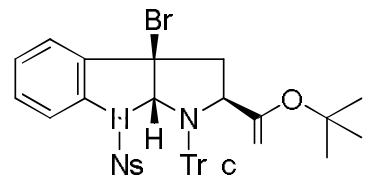

Purified by flash chromatography (hexanes:EtOAc from 80:20 to $70: 30$ ) $59 \%$ yield; exo.

${ }^{1} \mathrm{HRMN}\left(400 \mathrm{MHz}, \mathrm{CDCl}_{3}\right)$ : $\delta(\mathrm{ppm}) 1.45(\mathrm{~s}, 9 \mathrm{H}) ; 2.85(\mathrm{dd}, J=10.5$ and $12.4 \mathrm{~Hz}, 1 \mathrm{H}) ; 3.08-3.28(\mathrm{~m}, 1 \mathrm{H}) ; 3.66-4.08$ $(\mathrm{m}, 1 \mathrm{H}) ; 4.22-4.67(\mathrm{~m}, 1 \mathrm{H}) ; 4.88-5.26(\mathrm{~m}, 1 \mathrm{H}) ; 6.59(\mathrm{~s}, 1 \mathrm{H}) ; 7.31(\mathrm{dd}, J=7.4$ and $7.5 \mathrm{~Hz}, 1 \mathrm{H}) ; 7.42-7.50(\mathrm{~m}, 3 \mathrm{H})$; 7.52-7.71 (m, 4H).

${ }^{13} \mathrm{CRMN}\left(400 \mathrm{MHz}, \mathrm{CDCl}_{3}\right): \delta(\mathrm{ppm}) 28.1(3 \mathrm{q}) ; 43.8(\mathrm{t}) ; 59.1$ (s); 60.7 (d); 74.9 (t); 83.1 (s); 85.5 (d); 95.2 (s); 119.9 (d); 124.2 (d); 124.4 (2d); 127.6 (d); 130.3 (s); 131.6 (2d); 134.5 (d); 137.9 (s); 140.0 (s); 149.0 (s); 167.8 (s); $168.6(\mathrm{~s})$.

IR (KBr): v $\left(\mathrm{cm}^{-1}\right)$ 2980, 1741, 1546, 1464, 1371, 1218, 1152, 1062, 851, 773, 595.

HRMS (ESI+) calculated for $\mathrm{C}_{24} \mathrm{H}_{24} \mathrm{BrCl}_{3} \mathrm{~N}_{3} \mathrm{O}_{8} \mathrm{~S} \mathrm{~m} / z\left(\mathrm{M}+\mathrm{H}^{+}\right)$697.9533, found 697.9526 . 


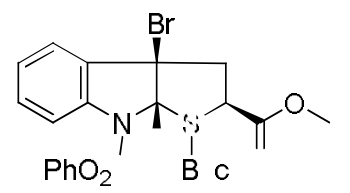

Purified by flash chromatography (hexanes:EtOAc 70:30); 92\% yield.

${ }^{1} \mathrm{H}-\mathrm{RMN}\left(400 \mathrm{MHz}, \mathrm{CDCl}_{3}\right.$ ): $\delta$ (ppm) 1.54 (bs, 9H); 2.77 (dd, $J=10.4$ and $12.6 \mathrm{~Hz}, 1 \mathrm{H}$ ); 3.06 (dd, $J=6.0$ and 12.6 $\mathrm{Hz}, 1 \mathrm{H}) ; 3.73(\mathrm{~s}, 3 \mathrm{H}) ; 3.83(\mathrm{dd}, J=6.0$ and $10.4 \mathrm{~Hz}, 1 \mathrm{H}) ; 6.32(\mathrm{bs}, 1 \mathrm{H}) ; 7.17$ (dd, $J=7.5$ and $8.0 \mathrm{~Hz}, 1 \mathrm{H}$ ); $7.24-$ $7.28(\mathrm{~m}, 1 \mathrm{H}) ; 7.34(\mathrm{dd}, J=7.1$ and $7.5 \mathrm{~Hz}, 3 \mathrm{H}) ; 7.46(\mathrm{t}, \mathrm{J}=7.5 \mathrm{~Hz}, 1 \mathrm{H}) ; 7.57(\mathrm{~d}, J=8.0 \mathrm{~Hz}, 1 \mathrm{H}) ; 7.82(\mathrm{bs}, 2 \mathrm{H})$.

${ }^{13} \mathrm{C}-\mathrm{RMN}\left(100 \mathrm{MHz}, \mathrm{CDCl}_{3}\right.$ ): $\delta$ (ppm) 28.3 (3q); 43.2 (t); 52.5 (q); 58.6 (s); 59.5 (d); 86.7 (d); 88.9 (s); 118.8 (d); 123.9 (d); 126.4 (d); 128.3 (2d); 128.6 (2d); 129.3 (s); 130.9 (d); 133.4 (d); 134.1 (s); 140.3 (s); 170.8 (s).

IR (KBr): v $\left(\mathrm{cm}^{-1}\right)$ 2979, 1752, 1702, 1448, 1367, 1032, 1171, 733.

HRMS (ESI+) calculated for $\mathrm{C}_{23} \mathrm{H}_{26} \mathrm{BrN}_{2} \mathrm{O}_{6} \mathrm{~S} \mathrm{~m} / z\left(\mathrm{M}+\mathrm{H}^{+}\right)$537.0689, found 537.0689.

exo-Methyl 8-benzenesulfonyl-1-benzyloxycarbonyl-3a-bromo-1,2,3,3a,8,8a-hexahydropyrrolo[2,3b]indole-2-carboxylate (3j)<smiles>COC(=O)C1CC(Br)(Br)C1(I)c1ccccc1</smiles>

$\mathrm{PhO}_{2} \quad \mathrm{Cbz}$

Purified by flash chromatography (hexanes:EtOAc 70:30); $82 \%$ yield.

${ }^{1} \mathrm{H}-\mathrm{RMN}\left(400 \mathrm{MHz}, \mathrm{CDCl}_{3}\right): \delta(\mathrm{ppm}) 2.78(\mathrm{dd}, J=10.1$ and $12.8 \mathrm{~Hz}, 1 \mathrm{H}) ; 3.09$ (dd, $J=6.3$ and $12.8 \mathrm{~Hz}, 1 \mathrm{H}$ ); 3.68 (bs, $3 \mathrm{H}) ; 3.88(\mathrm{dd}, J=6.3$ and $10.1 \mathrm{~Hz}, 1 \mathrm{H}) ; 5.13-5.45(\mathrm{~m}, 2 \mathrm{H}) ; 6.33(\mathrm{bs}, 1 \mathrm{H}) ; 7.18(\mathrm{dd}, J=7.4$ and $8.0 \mathrm{~Hz}, 1 \mathrm{H})$; $7.26(\mathrm{~d}, J=6.8 \mathrm{~Hz}, 2 \mathrm{H}) ; 7.29-7.40(\mathrm{~m}, 6 \mathrm{H}) ; 7.47(\mathrm{t}, J=7.4 \mathrm{~Hz}, 2 \mathrm{H}) ; 7.62(\mathrm{~d}, J=8.1 \mathrm{~Hz}, 1 \mathrm{H}) ; 7.79$ (bs, $2 \mathrm{H})$.

${ }^{13} \mathrm{C}-\mathrm{RMN}\left(100 \mathrm{MHz}, \mathrm{CDCl}_{3}\right)$ : $\delta(\mathrm{ppm}) 43.3$ (t); 52.4 (q); 59.3 (d); 59.5 (s); 67.9 (t); 86.7 (d); 118.9 (d); 123.8 (2d); 126.4 (d); 128.2 (2d); 128.3 (2d); 128.7 (2d); 128.8 (2d and s); 131.0 (d); 133.5 (d); 133.8 (s); 138.4 (s); 140.2 (s); $170.4(\mathrm{~s})$.

IR (KBr): v $\left(\mathrm{cm}^{-1}\right)$ 2953, 1749, 1711, 1408, 1366, 1172, 1029, 755.

HRMS (ESI+) calculated for $\mathrm{C}_{26} \mathrm{H}_{24} \mathrm{BrN}_{2} \mathrm{O}_{6} \mathrm{~S} \mathrm{~m} / z\left(\mathrm{M}+\mathrm{H}^{+}\right) 571.0533$, found 571.0531 .

exo-tert-Butyl 1-benzyloxycarbonyl-3a-bromo-8-benzenesulfonyl-1,2,3,3a,8,8a-hexahydropyrrolo[2,3b]indole-2-carboxylate (3k).

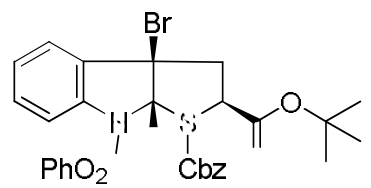

Purified by flash chromatography (hexanes/EtOAc from 80:20 to $70: 30$ ) $83 \%$ yield; exo.

${ }^{1} \mathrm{H}-\mathrm{RMN}\left(400 \mathrm{MHz}, \mathrm{CDCl}_{3}\right): \delta(\mathrm{ppm}) 1.35(\mathrm{~s}, 9 \mathrm{H}) ; 2.73(\mathrm{dd}, J=10.2$ and $12.7 \mathrm{~Hz}, 1 \mathrm{H}) ; 3.09$ (dd, $J=6.3$ and 12.7 $\mathrm{Hz}, 1 \mathrm{H}) ; 3.78(\mathrm{bs}, 1 \mathrm{H}) ; 5.11(\mathrm{~d}, J=12.1,1 \mathrm{H}) ; 5.37$ (bs, $1 \mathrm{H}) ; 6.31$ (bs, $1 \mathrm{H}) ; 7.17(\mathrm{t}, J=7.6 \mathrm{~Hz}, 1 \mathrm{H}) ; 7.25-7.40(\mathrm{~m}$, $7 \mathrm{H}) ; 7.46(\mathrm{t}, J=7.4 \mathrm{~Hz}, 2 \mathrm{H}) ; 7.47-7.51(\mathrm{~m}, 1 \mathrm{H}) ; 7.60(\mathrm{~d}, J=8.1 \mathrm{~Hz}, 1 \mathrm{H}) ; 7.79$ (bs, 2H).

${ }^{13} \mathrm{C}-\mathrm{RMN}\left(100 \mathrm{MHz}, \mathrm{CDCl}_{3}\right)$ : $\delta(\mathrm{ppm}) 27.7(3 \mathrm{q}) ; 43.3(\mathrm{t}) ; 59.3(\mathrm{~s}) ; 60.2$ (d); 67.6 (t); 82.2 (s); 86.9 (d); 118.8 (d); 123.9 (2d); 126.3 (d); 128.0 (d); 128.2 (d); 128.3 (2d); 128.7 (2d); 129.4 (s); 130.9 (2d); 133.4 (2d); 134.1 (s); $138.5(\mathrm{~s}) ; 140.2(\mathrm{~s}) ; 161.9(\mathrm{~s}) ; 169.0(\mathrm{~s})$.

IR (KBr): v $\left(\mathrm{cm}^{-1}\right)$ 2978, 1715, 1463, 1368, 1216, 1172, 1041, 595.

HRMS (ESI+) calculated for $\mathrm{C}_{29} \mathrm{H}_{30} \mathrm{BrN}_{2} \mathrm{O}_{6} \mathrm{~S} \mathrm{~m} / z\left(\mathrm{M}+\mathrm{H}^{+}\right) 613.1008$, found 613.1002 .

exo-Methyl 8-benzenesulfonyl-3a-bromo-1-methoxycarbonyl-1,2,3,3a,8,8a-hexahydropyrrolo[2,3-b]indole2-carboxylate (3I).

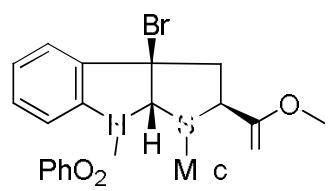

Purified by flash chromatography (hexanes:EtOAc 70:30); 96\% yield. 
${ }^{1} \mathrm{H}-\mathrm{RMN}\left(400 \mathrm{MHz}, \mathrm{CDCl}_{3}\right): \delta(\mathrm{ppm}) 2.81$ (dd, $J=10.4$ and $\left.12.7 \mathrm{~Hz}, 1 \mathrm{H}\right) ; 3.11$ (dd, $J=6.0$ and $12.7 \mathrm{~Hz}, 1 \mathrm{H}$ ); 3.75 (s, 3H); 3.81 (bs, $3 \mathrm{H}) ; 3.87$ (dd, $J=6.0$ and $10.4 \mathrm{~Hz}, 1 \mathrm{H}) ; 6.29(\mathrm{~s}, 1 \mathrm{H}) ; 7.18(\mathrm{dd}, J=7.4$ and $7.7 \mathrm{~Hz}, 1 \mathrm{H}) ; 7.27$ (bd, $\mathrm{J}=7.7 \mathrm{~Hz}, 1 \mathrm{H}) ; 7.31-7.39(\mathrm{~m}, 3 \mathrm{H}) ; 7.48(\mathrm{dd}, J=7.4$ and $7.5 \mathrm{~Hz}, 1 \mathrm{H}) ; 7.60(\mathrm{~d}, J=8.2 \mathrm{~Hz}, 1 \mathrm{H}) ; 7.80(\mathrm{~d}, J=7.4 \mathrm{~Hz}$, $2 \mathrm{H})$.

${ }^{13} \mathrm{C}-\mathrm{RMN}\left(100 \mathrm{MHz}, \mathrm{CDCl}_{3}\right.$ ): $\delta$ (ppm) 43.4 (t); 52.7 (q); 53.0 (q); 59.1 (s); 59.2 (d); 86.7 (d); 118.8 (d); 123.8 (d); 126.4 (d); 128.1 (2d); 128.8 (2d); 131.0 (d); 133.5 (d); 133.7 (s); 138.4 (s); 140.2 (s); 149.3 (s); 170.4 (s).

IR (KBr): v $\left(\mathrm{cm}^{-1}\right)$ 2954, 1714, 1447, 1366, 1173, 1092, 1027, 733.

HRMS (ESI+) calculated for $\mathrm{C}_{20} \mathrm{H}_{20} \mathrm{BrN}_{2} \mathrm{O}_{6} \mathrm{~S} \mathrm{~m} / \mathrm{z}\left(\mathrm{M}+\mathrm{H}^{+}\right)$495.0220, found 495.0219.

\section{endo-tert-Butyl 8-benzenesulfonyl-3a-bromo-1-methoxycarbonyl-1,2,3,3a,8,8a-hexahydropyrrolo[2,3-}

b]indole-2-carboxylate $(3 \mathrm{~m})$

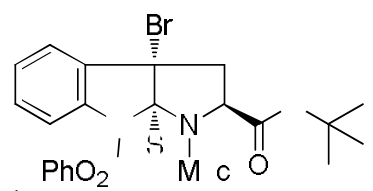

${ }^{1} \mathrm{H}-\mathrm{RMN}\left(400 \mathrm{MHz}, \mathrm{CDCl}_{3}\right): \delta(\mathrm{ppm}) 1.08(\mathrm{~s}, 9 \mathrm{H}) ; 3.04(\mathrm{dd}, J=9.6$ and $13.2 \mathrm{~Hz}, 1 \mathrm{H}) ; 3.23(\mathrm{~d}, J=13.2 \mathrm{~Hz}, 1 \mathrm{H})$; $3.65(\mathrm{~s}, 3 \mathrm{H}) ; 4.49(\mathrm{~d}, J=9.6 \mathrm{~Hz}, 1 \mathrm{H}) ; 6.35(\mathrm{~s}, 1 \mathrm{H}) ; 7.12(\mathrm{dd}, J=7.5$ and $7.8 \mathrm{~Hz}, 1 \mathrm{H}) ; 7.27-7.35(\mathrm{~m}, 2 \mathrm{H}) ; 7.41(\mathrm{t}, J$ $=7.6 \mathrm{~Hz}, 2 \mathrm{H}) ; 7.47-7.55(\mathrm{~m}, 2 \mathrm{H}) ; 7.86(\mathrm{~d}, J=7.6 \mathrm{~Hz}, 2 \mathrm{H})$.

${ }^{13} \mathrm{C}-\mathrm{RMN}\left(100 \mathrm{MHz}, \mathrm{CDCl}_{3}\right.$ ): $\delta(\mathrm{ppm}) 27.5(3 \mathrm{q}) ; 44.3(\mathrm{t}) ; 52.7$ (s); 52.9 (q); 60.2 (d); 82.0 (s); 87.2 (d); 118.2 (d); 124.5 (d); 125.9 (d); 127.4 (2d); 128.9 (2d); 129.1 (s); 131.0 (d); 133.2 (d); 133.6 (s); 141.6 (s); 157.2 (s); 160.0 (s). IR (KBr): v $\left(\mathrm{cm}^{-1}\right)$ 2979, 1721, 1600, 1447, 1369, 1172, 1092, 975, 850, 756, 735.

HRMS (ESI+) calculated for $\mathrm{C}_{23} \mathrm{H}_{26} \mathrm{BrN}_{2} \mathrm{O}_{6} \mathrm{~S} \mathrm{~m} / \mathrm{z}\left(\mathrm{M}+\mathrm{H}^{+}\right)$537.0689, found 537.0687.

exo-tert-Butyl 8-benzenesulfonyl-3a-bromo-1-methoxycarbonyl-1,2,3,3a,8,8a-hexahydropyrrolo[2,3b]indole-2-carboxylate $(3 \mathrm{~m})$.

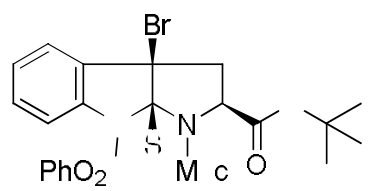

Purified by flash chromatography (hexanes/EtOAc from 80:20 to 70:30) 58\% yield; endo:exo (25:75).

${ }^{1} \mathrm{H}-\mathrm{RMN}\left(400 \mathrm{MHz}, \mathrm{CDCl}_{3}\right)$ : exo product $\delta(\mathrm{ppm}) 1.45(\mathrm{~s}, 9 \mathrm{H}) ; 2.75(\mathrm{dd}, J=10.3$ and $12.7 \mathrm{~Hz}, 1 \mathrm{H}) ; 3.09(\mathrm{dd}, J=$ 6.3 and $12.7 \mathrm{~Hz}, 1 \mathrm{H}) ; 3.75(\mathrm{dd}, J=6.3$ and $10.3 \mathrm{~Hz}, 1 \mathrm{H}) ; 3.78(\mathrm{bs}, 3 \mathrm{H}) ; 6.28(\mathrm{~s}, 1 \mathrm{H}) ; 7.18(\mathrm{t}, J=7.6 \mathrm{~Hz}, 1 \mathrm{H}) ; 7.29$ (t, $J=7.0 \mathrm{~Hz}, 1 \mathrm{H}) ; 7.32-7.40(\mathrm{~m}, 3 \mathrm{H}) ; 7.42-7.51(\mathrm{~m}, 1 \mathrm{H}) ; 7.58(\mathrm{~d}, J=8.1 \mathrm{~Hz}, 1 \mathrm{H}) ; 7.79(\mathrm{bd}, J=6.9 \mathrm{~Hz}, 2 \mathrm{H})$.

${ }^{13} \mathrm{C}-\mathrm{RMN}\left(100 \mathrm{MHz}, \mathrm{CDCl}_{3}\right)$ : exo product $\delta(\mathrm{ppm}) 27.8(\mathrm{q}) ; 43.6(\mathrm{t}) ; 52.8(\mathrm{q}) ; 59.2$ (s); 60.1 (d); 82.3 (s); 86.9 (d); 118.7 (d); 123.9 (d); 125.1 (s); 126.3 (d); 128.1 (2d); 128.7 (d); 129.4 (d); 130.9 (d); 133.4 (d); 138.5 (s); 140.2 (s); $169.6(\mathrm{~s}) ; 171.5(\mathrm{~s})$.

IR (KBr): v $\left(\mathrm{cm}^{-1}\right)$ 2979, 1725, 1447, 1369, 1172, 595.

HRMS (ESI+) calculated for $\mathrm{C}_{23} \mathrm{H}_{26} \mathrm{BrN}_{2} \mathrm{O}_{6} \mathrm{~S} \mathrm{~m} / \mathrm{z}\left(\mathrm{M}+\mathrm{H}^{+}\right)$537.0695, found 537.0681 .

exo-Methyl 2-( $N^{a}$-allyloxycarbonyl-Ala-O-yl)-3a-bromo-1-(tert-butoxycarbonyl)-1,2,3,3a,8,8ahexahydropyrrolo[2,3-b]indole-2-carboxylate (3n)

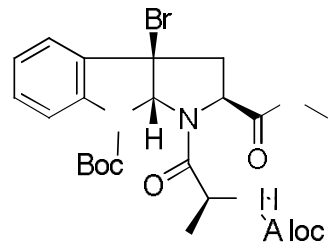

Purified by flash chromatography (hexanes/EtOAc from 80:20 to $70: 30$ ) $47 \%$ yield; exo.

${ }^{1} \mathrm{H}-\mathrm{RMN}\left(400 \mathrm{MHz}, \mathrm{CDCl}_{3}\right): \delta(\mathrm{ppm}) 1.50(\mathrm{~d}, J=6.8 \mathrm{~Hz}, 3 \mathrm{H}) ; 1.62(\mathrm{~s}, 9 \mathrm{H}) ; 2.79$ (dd, $J=10.9$ and $\left.12.5 \mathrm{~Hz}, 1 \mathrm{H}\right)$; $3.26(\mathrm{dd}, J=6.2$ and $12.5 \mathrm{~Hz}, 1 \mathrm{H}) ; 3.73(\mathrm{~s}, 3 \mathrm{H}) ; 4.03(\mathrm{dd}, \mathrm{J}=6.2$ and $10.9 \mathrm{~Hz}, 1 \mathrm{H}) ; 4.46-4.57(\mathrm{~m}, 2 \mathrm{H}) ; 5.02(\mathrm{t}, 6.8$ $\mathrm{Hz}, 1 \mathrm{H}) ; 5.13-5.29(\mathrm{~m}, 2 \mathrm{H}) ; 5.43(\mathrm{bs}, 1 \mathrm{H}) ; 5.82-5.94(\mathrm{~m}, 1 \mathrm{H}) ; 6.41(\mathrm{~s}, 1 \mathrm{H}) ; 7.18(\mathrm{dd}, J=7.3$ and $7.4 \mathrm{~Hz}, 1 \mathrm{H}) ; 7.32-$ $7.40(\mathrm{~m}, 2 \mathrm{H}) ; 7.50(\mathrm{bs}, 1 \mathrm{H})$.

${ }^{13} \mathrm{C}-\mathrm{RMN}\left(100 \mathrm{MHz}, \mathrm{CDCl}_{3}\right)$ : $\delta(\mathrm{ppm}) 19.3$ (q); 28.1 (3q); 40.6 (t); 47.9 (d); 52.5 (q); 60.0 (d); 65.3 (t); 67.9 (s); 84.0 (s); 84.5 (d); 117.3 (t); 120.5 (d); 122.8 (d); 125.6 (d); 128.1 (s); 130.8 (d), 132.9 (d); 133.4 (s); 154.9 (s); 170.3 (s); $173.8(\mathrm{~s})$. 
IR (KBr): v $\left(\mathrm{cm}^{-1}\right)$ 3325, 2979, 1724, 1663, 1478, 1414, 1370, 1333, 1254, 1155, 852, 754.

HRMS (ESI+) calculated for $\mathrm{C}_{24} \mathrm{H}_{31} \mathrm{BrN}_{3} \mathrm{O}_{7} \mathrm{~m} / z\left(\mathrm{M}+\mathrm{H}^{+}\right)$552.1340, found 552.1338.

exo-Methoxymethyloxyisoleucinyl 2-allyloxycarbonyl-3a-bromo-1-(tert-butoxycarbonyl)-1,2,3,3a,8,8ahexahydropyrrolo[2,3-b]indole-2-carbonylate (30)<smiles>C=C(OCOC)C(NC(=O)C1CC(Br)(Br)C12[B]c1ccccc12)C(C)CC</smiles>

${ }^{1} \mathrm{H}-\mathrm{RMN}\left(400 \mathrm{MHz}, \mathrm{CDCl}_{3}\right): \delta(\mathrm{ppm}) 0.91$ (t, $\left.J=7.5 \mathrm{~Hz}, 3 \mathrm{H}\right) ; 0.93(\mathrm{~d}, J=6.9 \mathrm{~Hz}, 3 \mathrm{H}) ; 1.14-1.24(\mathrm{~m}, 1 \mathrm{H}) ; 1.39-1.49$ $(\mathrm{m}, 1 \mathrm{H}) ; 1.57(\mathrm{~s}, 9 \mathrm{H}) ; 1.88-1.98(\mathrm{~m}, 1 \mathrm{H}) ; 2.93(\mathrm{dd}, J=10.1$ and $12.8 \mathrm{~Hz}, 1 \mathrm{H}) ; 3.22(\mathrm{dd}, J=6.4$ and $12.8 \mathrm{~Hz}, 1 \mathrm{H})$; $3.47(\mathrm{~s}, 3 \mathrm{H}) ; 3.85(\mathrm{dd}, J=6.4$ and $10.1 \mathrm{~Hz}, 1 \mathrm{H}) ; 4.48-4.65(\mathrm{~m}, 3 \mathrm{H}) ; 5.12-5.24(\mathrm{~m}, 2 \mathrm{H}) ; 5.20(\mathrm{~d}, J=5.9 \mathrm{~Hz}, 1 \mathrm{H})$; $5.33(\mathrm{~d}, J=5.9 \mathrm{~Hz}, 1 \mathrm{H}) ; 5.76-5.88(\mathrm{~m}, 1 \mathrm{H}) ; 6.23(\mathrm{~d}, J=8.5 \mathrm{~Hz}, 1 \mathrm{H}) ; 6.39(\mathrm{~s}, 1 \mathrm{H}) ; 7.12(\mathrm{dd}, J=7.5$ and $8.3 \mathrm{~Hz}$, $1 \mathrm{H}) ; 7.31(\mathrm{dd}, J=7.2$ and $8.3 \mathrm{~Hz}, 1 \mathrm{H}) ; 7.36(\mathrm{~d}, J=7.5 \mathrm{~Hz}, 1 \mathrm{H}) ; 7.58(\mathrm{bd}, J=7.2 \mathrm{~Hz}, 1 \mathrm{H})$.

${ }^{13} \mathrm{C}-\mathrm{RMN}\left(100 \mathrm{MHz}, \mathrm{CDCl}_{3}\right): \delta(\mathrm{ppm}) 11.6(\mathrm{q}) ; 15.3(\mathrm{q}) ; 25.0(\mathrm{t}) ; 28.2(3 \mathrm{q}) ; 37.8(\mathrm{~d}) ; 41.9(\mathrm{t}) ; 56.6(\mathrm{~d}) ; 57.9(\mathrm{q}) ; 59.8$ (s); 61.2 (d); $66.3(\mathrm{t}) ; 82.3(\mathrm{~s}) ; 84.1$ (d); $91.1(\mathrm{t}) ; 117.5$ (t); 118.3 (d); 123.2 (d); 124.4 (d); 130.6 (d); 132.3 (d); 132.8 (s); $141.2(\mathrm{~s}) ; 152.0(\mathrm{~s}) ; 169.7$ (s); $171.4(2 \mathrm{~s})$.

IR (KBr): v $\left(\mathrm{cm}^{-1}\right)$ 3333, 2967, 2934, 1718, 1540, 1478, 1369, 1335, 1161, 927, 755.

HRMS (ESI+) calculated for $\mathrm{C}_{28} \mathrm{H}_{38} \mathrm{BrN}_{3} \mathrm{O}_{8} \mathrm{Na} \mathrm{m} / z\left(\mathrm{M}+\mathrm{Na}^{+}\right) 646.1740$, found 646.1730.

exo-Allyloxyisoleucinyl 3a-bromo-1,8-(di-tert-butoxycarbonyl)-1,2,3,3a,8,8a-hexahydropyrrolo[2,3-b]indole2-carbonylate (3p)

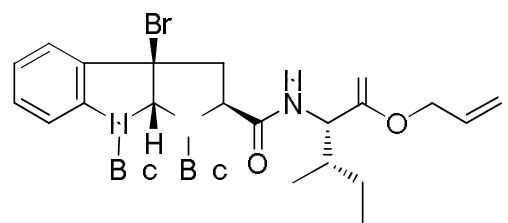

${ }^{1} \mathrm{H}-\mathrm{RMN}\left(400 \mathrm{MHz}, \mathrm{CDCl}_{3}\right): \delta(\mathrm{ppm})$ 0.88-0.94 (m, 6H); 1.15-1.26 (m, 1H); $1.39(\mathrm{~s}, 9 \mathrm{H}) ; 1.41-1.49(\mathrm{~m}, 1 \mathrm{H}) ; 1.58(\mathrm{~s}$, $9 \mathrm{H}) ; 1.86-1.93(\mathrm{~m}, 1 \mathrm{H}) ; 2.84-2.95(\mathrm{~m}, 1 \mathrm{H}) ; 3.18(\mathrm{dd}, J=6.3$ and $12.8 \mathrm{~Hz}, 1 \mathrm{H}) ; 3.77(\mathrm{dd}, J=6.3$ and $10.1 \mathrm{~Hz}, 1 \mathrm{H})$; 4.58 (dd, $J=3.6$ and $4.9 \mathrm{~Hz}, 1 \mathrm{H}$ ); 4.59-4.63 (m, 2H); 5.26 (ddd, $J=1.1,2.5$ and $10.4 \mathrm{~Hz}, 1 \mathrm{H}$ ); 5.33 (ddd, $J=1.4$, 2.5 and 17.2, $1 \mathrm{H}) ; 5.84-5.95(\mathrm{~m}, 1 \mathrm{H}) ; 6.28(\mathrm{~d}, J=8.5 \mathrm{~Hz}, 1 \mathrm{H}) ; 6.36(\mathrm{~s}, 1 \mathrm{H}) ; 7.12(\mathrm{t}, J=7.6 \mathrm{~Hz}, 1 \mathrm{H}) ; 7.30(\mathrm{dd}, J=$ 7.6 and $7.9 \mathrm{~Hz}, 1 \mathrm{H}) ; 7.35(\mathrm{bd}, J=7.6 \mathrm{~Hz}, 1 \mathrm{H}) ; 7.50(\mathrm{bd}, J=7.9 \mathrm{~Hz}, 1 \mathrm{H})$.

${ }^{13} \mathrm{C}-\mathrm{NMR}\left(100 \mathrm{MHz}, \mathrm{CDCl}_{3}\right) \delta(\mathrm{ppm}) 11.5$ (q); 15.4 (q); 25.1 (t); $28.2(3 \mathrm{q}) ; 28.3(3 \mathrm{q}) ; 38.1$ (d); 41.9 (t); 56.5 (d); 59.9 (s); 61.3 (d); 65.8 (t); 81.6 (s); 82.4 (s); 84.1 (d); 118.7 (d); 119.0 (t); 123.3 (d); 124.5 (d); 130.5 (d); 131.5 (d); 133.3 (s); 141.4 (s); 152.3 (s); 169.9 (s); $171.4(2 \mathrm{~s})$.

IR (KBr): v $\left(\mathrm{cm}^{-1}\right)$ 3343, 2975, 2933, 1721, 1532, 1478, 1394, 1368, 1331, 1165, 853, 751.

HRMS (ESI+) calculated for $\mathrm{C}_{30} \mathrm{H}_{42} \mathrm{BrN}_{3} \mathrm{O}_{7} \mathrm{~m} / \mathrm{z}(\mathrm{M})$ 635.2206, found 635.2628.

\section{Compound 5}

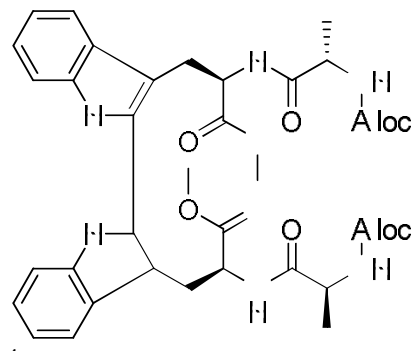

${ }^{1} \mathrm{H}-\mathrm{RMN}\left(400 \mathrm{MHz}, \mathrm{CDCl}_{3}\right): \delta(\mathrm{ppm}) 1.18(\mathrm{~s}, 3 \mathrm{H}) ; 1.20(\mathrm{~s}, 3 \mathrm{H}) ; 2.17-2.36(\mathrm{~m}, 2 \mathrm{H}) ; 3.24-3.41(\mathrm{~m}, 2 \mathrm{H}) ; 3.62(\mathrm{~s}, 3 \mathrm{H})$; $3.63(\mathrm{~s}, 3 \mathrm{H}) ; 3.78(\mathrm{bs}, 1 \mathrm{H}) ; 4.09(\mathrm{t}, J=7.1 \mathrm{~Hz}, 1 \mathrm{H}) ; 4.22-4.51(\mathrm{~m}, 5 \mathrm{H}) ; 4.70(\mathrm{dd}, J=7.6$ and $12.0 \mathrm{~Hz}, 1 \mathrm{H}) ; 4.92(\mathrm{dd}$, $J=6.7$ and $12.8 \mathrm{~Hz}, 1 \mathrm{H}) ; 5.13-5.28(\mathrm{~m}, 5 \mathrm{H}) ; 5.31(\mathrm{~d}, J=6.9 \mathrm{~Hz}, 1 \mathrm{H}) ; 5.49(\mathrm{~d}, J=6.0 \mathrm{~Hz}, 1 \mathrm{H}) ; 5.76-5.90(\mathrm{~m}, 2 \mathrm{H})$; $6.63(\mathrm{bs}, 1 \mathrm{H}) ; 6.95(\mathrm{bd}, J=6.4 \mathrm{~Hz}, 1 \mathrm{H}) ; 7.03-7.20(\mathrm{~m}, 5 \mathrm{H}) ; 7.22(\mathrm{~d}, J=8.0 \mathrm{~Hz}, 1 \mathrm{H}) ; 7.31(\mathrm{t}, J=4.4 \mathrm{~Hz}, 1 \mathrm{H}) ; 7.45$ $(\mathrm{d}, J=7.9 \mathrm{~Hz}, 2 \mathrm{H}) ; 9.42(\mathrm{~s}, 1 \mathrm{H})$.

${ }^{13} \mathrm{C}-\mathrm{RMN}\left(100 \mathrm{MHz}, \mathrm{CDCl}_{3}\right)$ : $\delta(\mathrm{ppm}) 17.4$ (q); 18.0 (q); 27.1 (t); 35.8 (t); 46.1 (d); 50.2 (d); 50.3 (2d); 52.6 (q); 52.7 (q); 52.9 (d); 60.6 (d); 66.0 (t); 66.2 (t); 109.2 (s); 111.7 (d); 116.0 (d); 118.1 (2t); 118.8 (d); 119.9 (d); 122.8 (s); 
123.2 (d); 124.7 (d); 126.1 (d); 127.6 (s); 127.9 (s); 129.1 (d); 132.1 (d); 132.3 (d); 136.1 (s); 156.4 (s); 160.9 (s); 161.2 (s); 171.8 (s); 172.9 (s); 173.1 (2s).

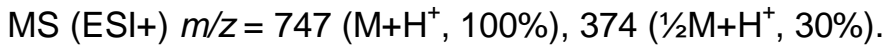

HPLC (MeCN: $\mathrm{H}_{2} \mathrm{O}$ des de 40:60 fins 50:50 en 8 minuts): $\mathrm{t}_{\mathrm{R}}=5.52 \mathrm{~min}$.

\section{Compound 8}

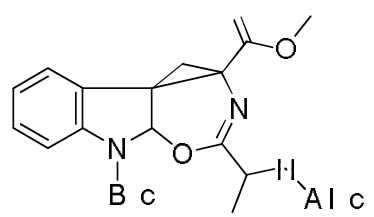

${ }^{1} \mathrm{H}-\mathrm{RMN}\left(400 \mathrm{MHz}, \mathrm{CDCl}_{3}\right): \delta(\mathrm{ppm}) 0.66$ and $0.77(2 \mathrm{~d}, J=6,6 \mathrm{~Hz}, 3 \mathrm{H}) ; 1.64(\mathrm{~s}, 9 \mathrm{H}) ; 3.43(\mathrm{~d}, J=15.4 \mathrm{~Hz}, 1 \mathrm{H})$; $3,81(\mathrm{~s}, 3 \mathrm{H}) ; 3.91(\mathrm{~d}, J=15.4 \mathrm{~Hz}, 1 \mathrm{H}) ; 4.04-4.19(\mathrm{~m}, 1 \mathrm{H}) ;$ 4.54-4.77 (m, 2H); 5.15-5.44 (m, 2H); 5.84-5.99 (m, $1 \mathrm{H})$; 7.08 (bs, $1 \mathrm{H}) ; 7.14$ (dd, $J=7.2$ and $7.6 \mathrm{~Hz}, 1 \mathrm{H}) ; 7.27(\mathrm{dd}, J=7.2$ and $8.2 \mathrm{~Hz}, 1 \mathrm{H}) ; 7.43-7.52(\mathrm{~m}, 2 \mathrm{H}) ; 8.08(\mathrm{~d}, J=$ $8.2 \mathrm{~Hz}, 1 \mathrm{H})$.

${ }^{13} \mathrm{C}-\mathrm{RMN}\left(100 \mathrm{MHz}, \mathrm{CDCl}_{3}\right): \delta(\mathrm{ppm}) 17.0$ (q); $28.1(3 \mathrm{q}) ; 28.9$ (t); 53.3 (q); 54.9 (d); 66.4 (t); 77.9 (s); 83.8 (s); 113.3 (s); 115.1 (d); 118.1 (t); 119.1 (d); 122.6 (d); 124.4 (d); 125.9 (d); 132.0 (s); 135.0 (s);149.6 (s); 154.0 (s); $170.2(\mathrm{~s}) ; 172.9(\mathrm{~s})$.

IR $(\mathrm{KBr}): \mathrm{v}\left(\mathrm{cm}^{-1}\right)$ 3283, 2931, 1735, 1452, 1370, 1257, 1158, 1082, 747.

HRMS (ESI+) calculated for $\mathrm{C}_{48} \mathrm{H}_{58} \mathrm{~N}_{6} \mathrm{O}_{14} \mathrm{Na} \mathrm{m} / \mathrm{z}\left(2 \mathrm{M}+\mathrm{Na}^{+}\right)$965.3909, found 965.3934 . 
1 a.

$\mathrm{H} 1 /$ s2pul/Mercury-400

Cdel3/Temp: 25 C/N reg: D-PCB010411092154<smiles></smiles>
Boc Álloc $\mathrm{O}$

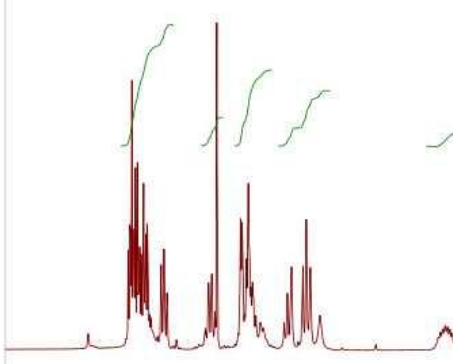
Whit

$\Upsilon$

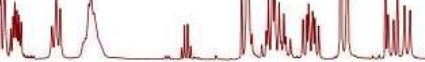

8.0

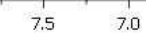

${ }_{\mathrm{f} 1 \text { (ppm) }}^{4.5}$

C13/s2pul/Mercury-400

cdcl3/Temp: $25^{\circ} \mathrm{C} / \mathrm{N}$ reg: N-PCB010411104558

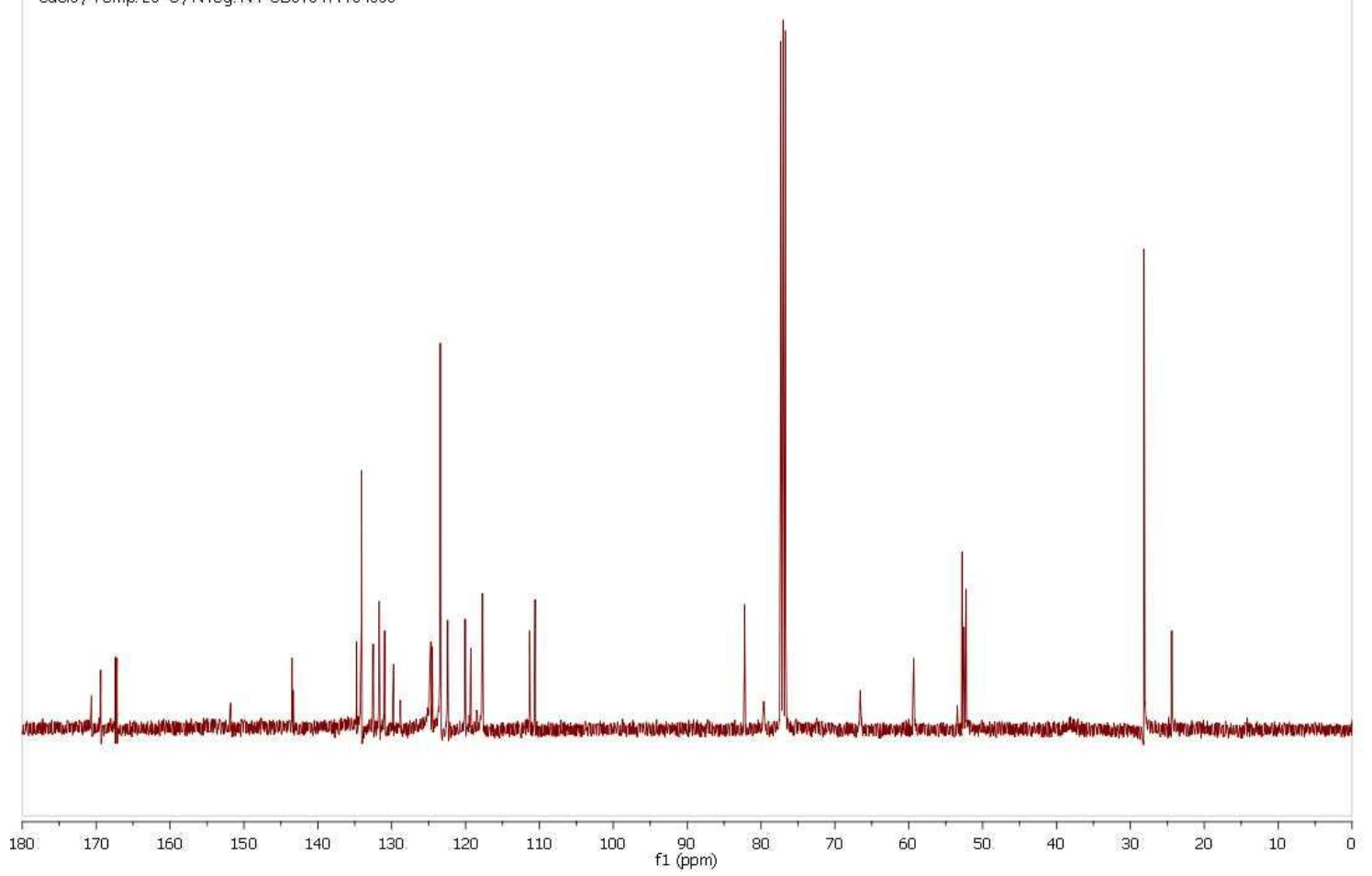


$1 b$.

$\mathrm{H1} /$ s2pul/Mercury-400

odc13/Temp: $25^{\circ} \mathrm{C} / \mathrm{N}$ reg: N-PCB180511092250<smiles>COC(=O)NC(Cc1cn(C23CC(C(=O)OC)N(C(=O)OC(C)(C)C)C2N(c2ccccc2)c2ccccc23)c2ccccc12)C(=O)OC</smiles>

Boc Boc O

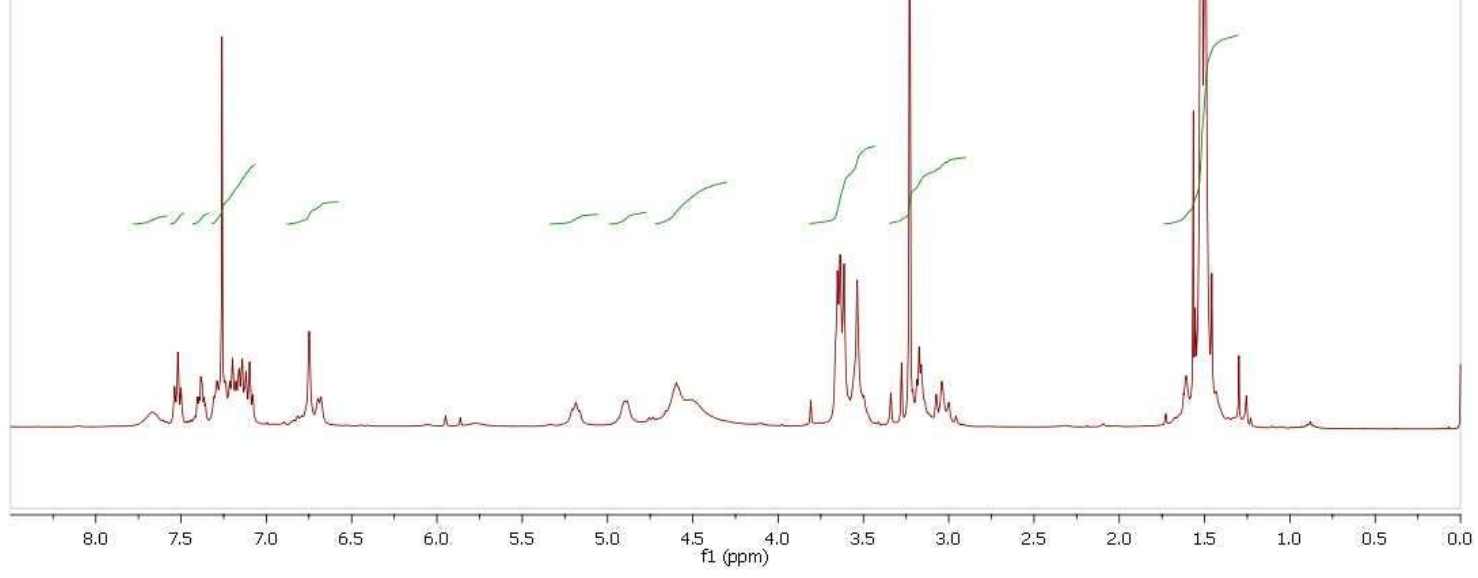

C13/s2pul/Mercury-40

odc13/Temp: $25^{\circ} \mathrm{C} / \mathrm{N}$ reg: N-PCB180511092250
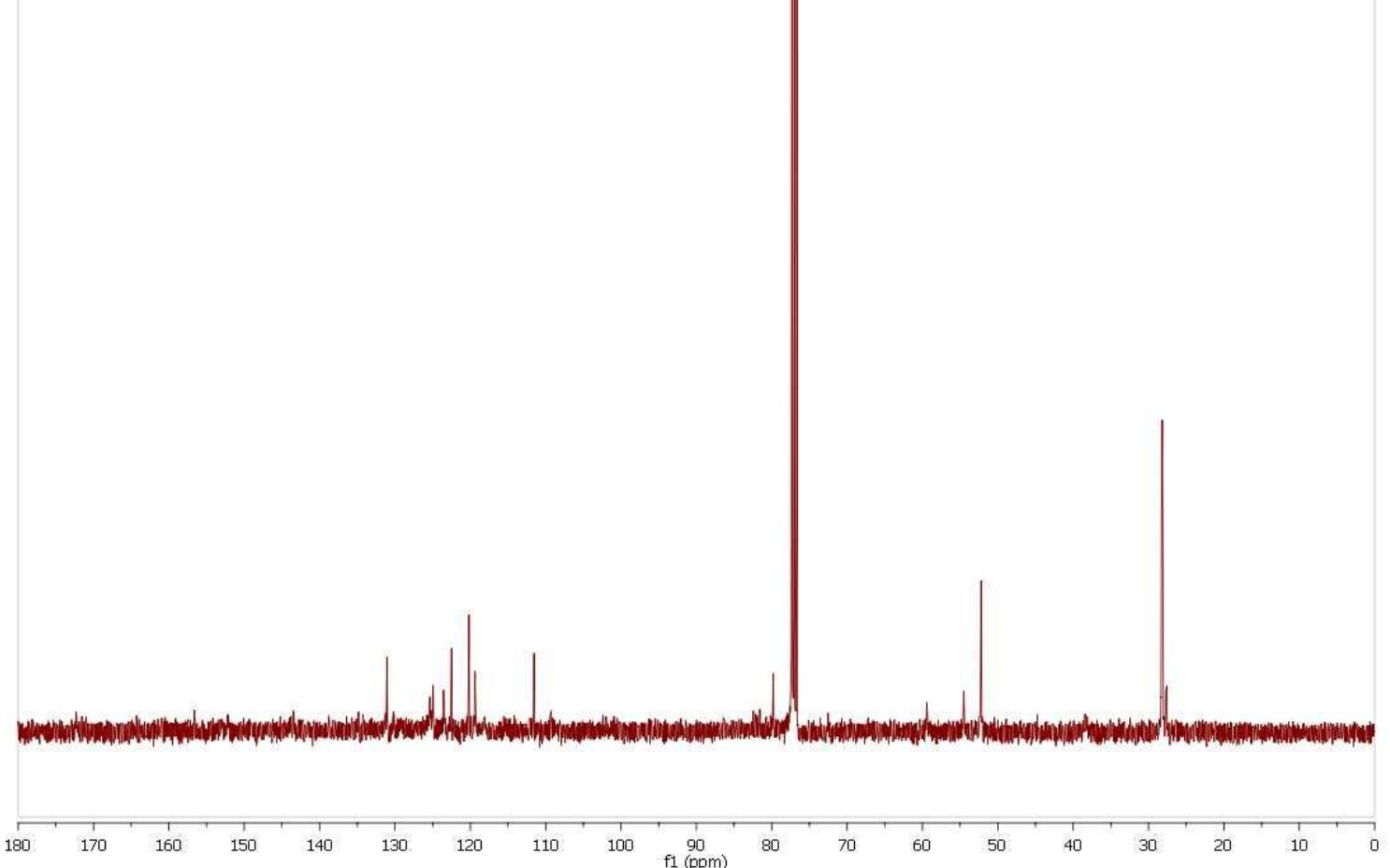
$1 c$.

$\mathrm{H} 1$ / s2pul/Mercury-400

cdcl3/Temp: $25^{\circ} \mathrm{C} / \mathrm{N}$ reg: D-PCB100309194703<smiles>C=C(OC)C1CC(c2ccccc2I)(n2cc(CC(NP)C(=O)CC)c3ccccc32)C(N)N1C</smiles>

$\mathrm{B}$ C $\mathrm{B}$ c

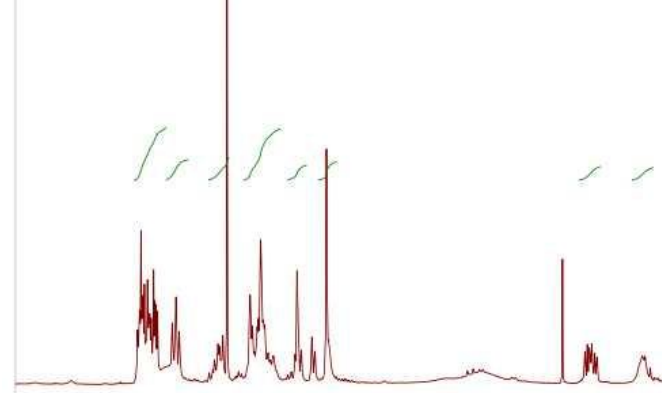

4
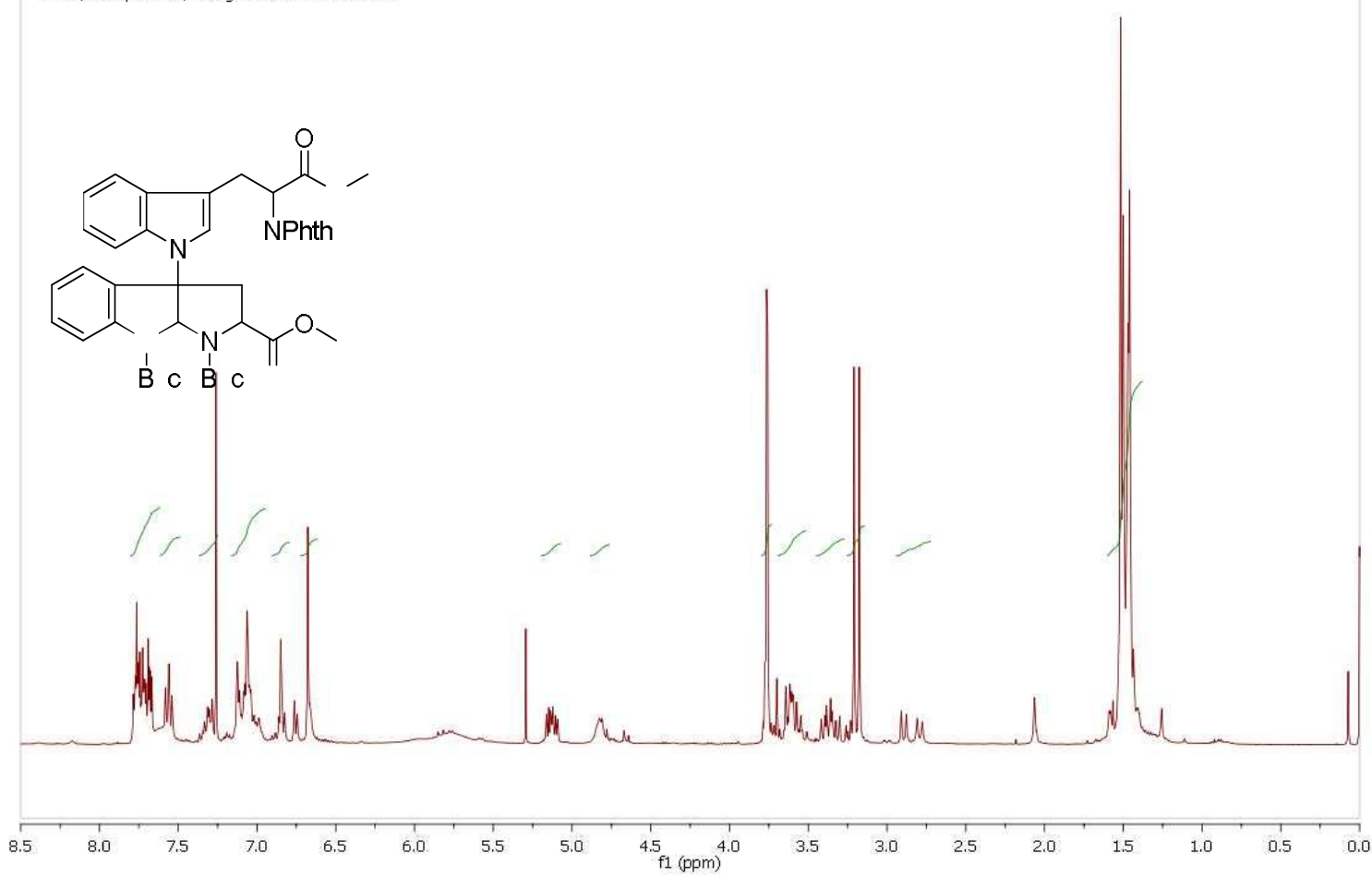

C13/s2pul/Mercury-400

cdcl $3 /$ Temp: $25^{\circ} \mathrm{C} / \mathrm{N}$ reg: D-PCB100309194703

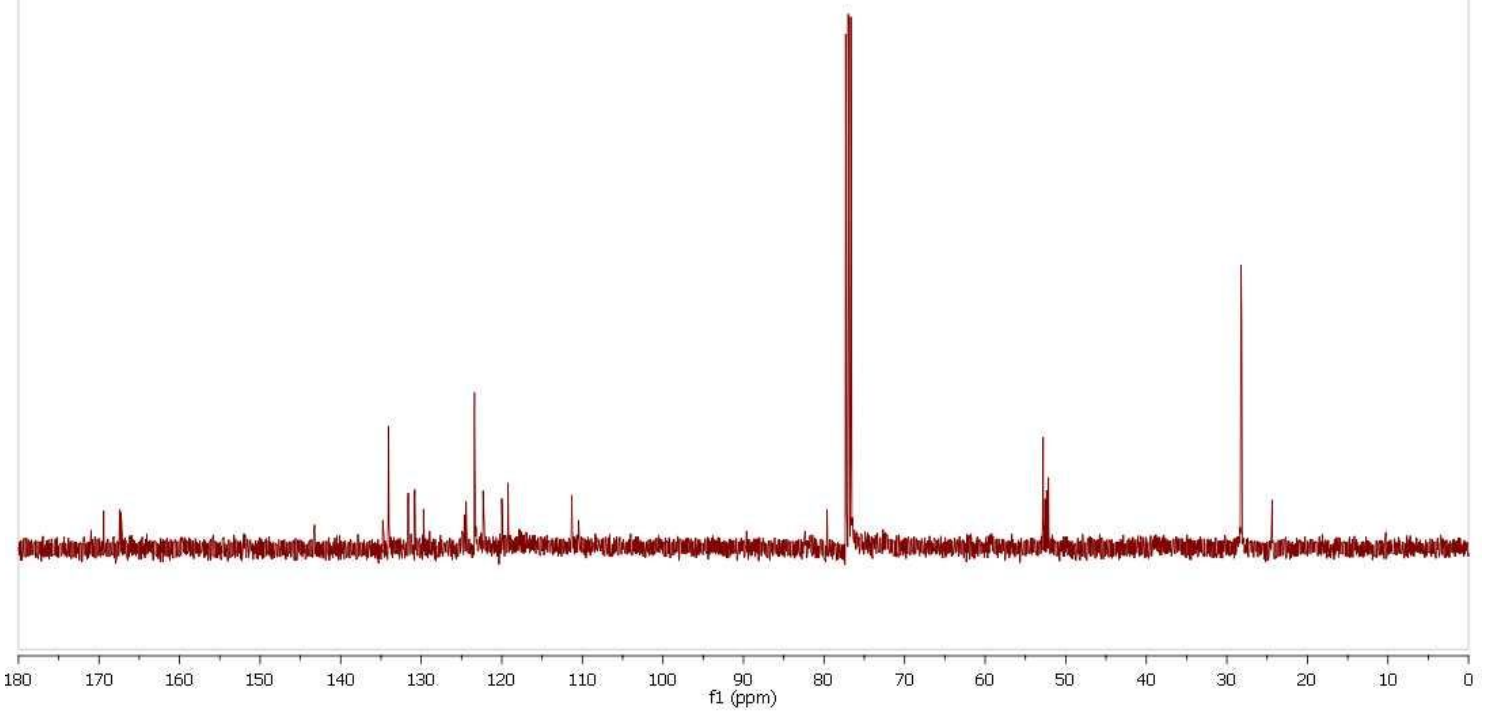

Create PDF files without this message by purchasing novaPDF printer (http://www.novapdf.com) 
$1 d$.

$\mathrm{H} 1 /$ s2pul / Mercury-400

cdcl $3 /$ Temp $: 25^{\circ} \mathrm{C} / \mathrm{N}$ reg: N-PCB060511083146<smiles></smiles>
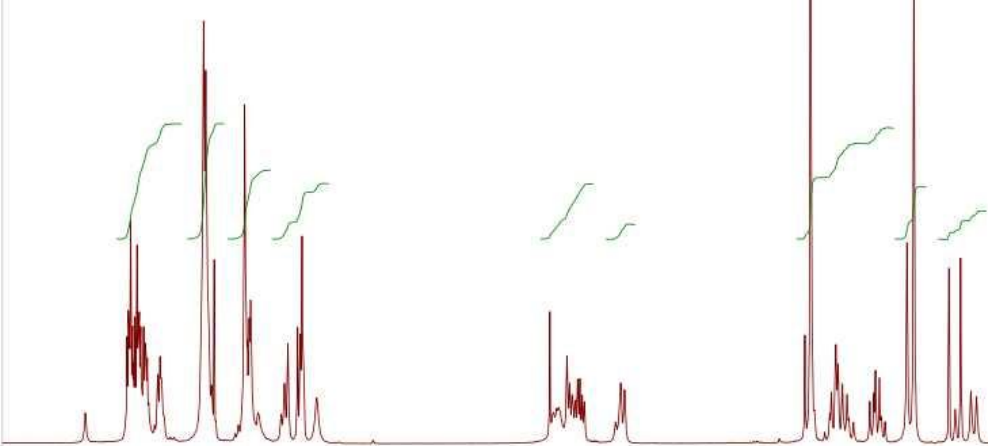

Nul

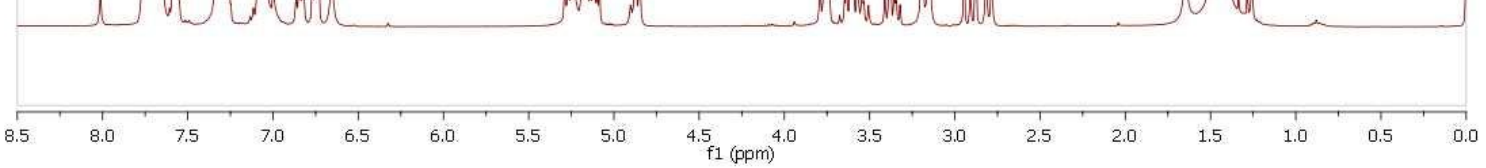

$\mathrm{C13} /$ s2pul/Mercury-400

cdcl3/Temp: $25^{\circ} \mathrm{C} / \mathrm{N}$ reg: N-PCB060511083146

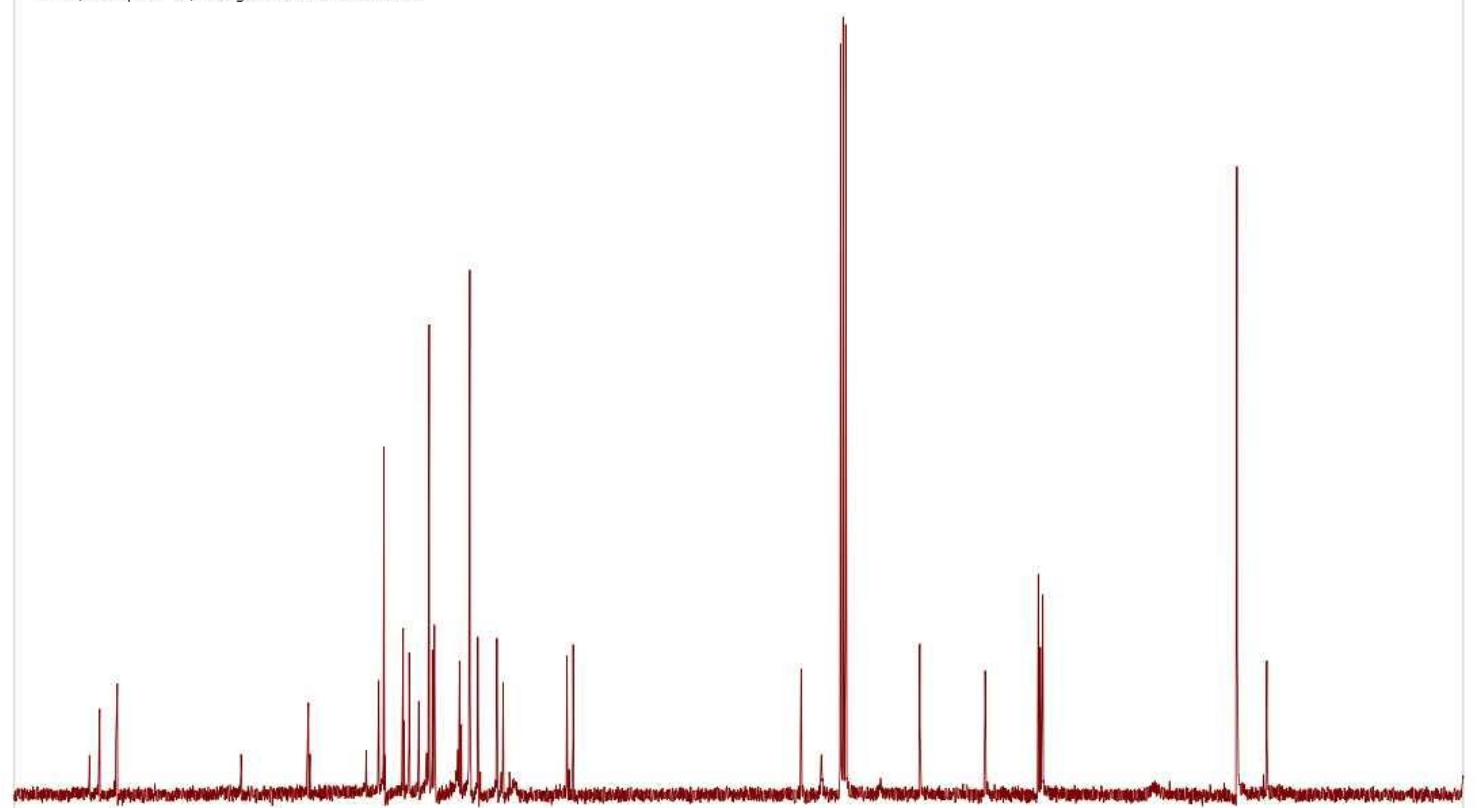


$1 e$.
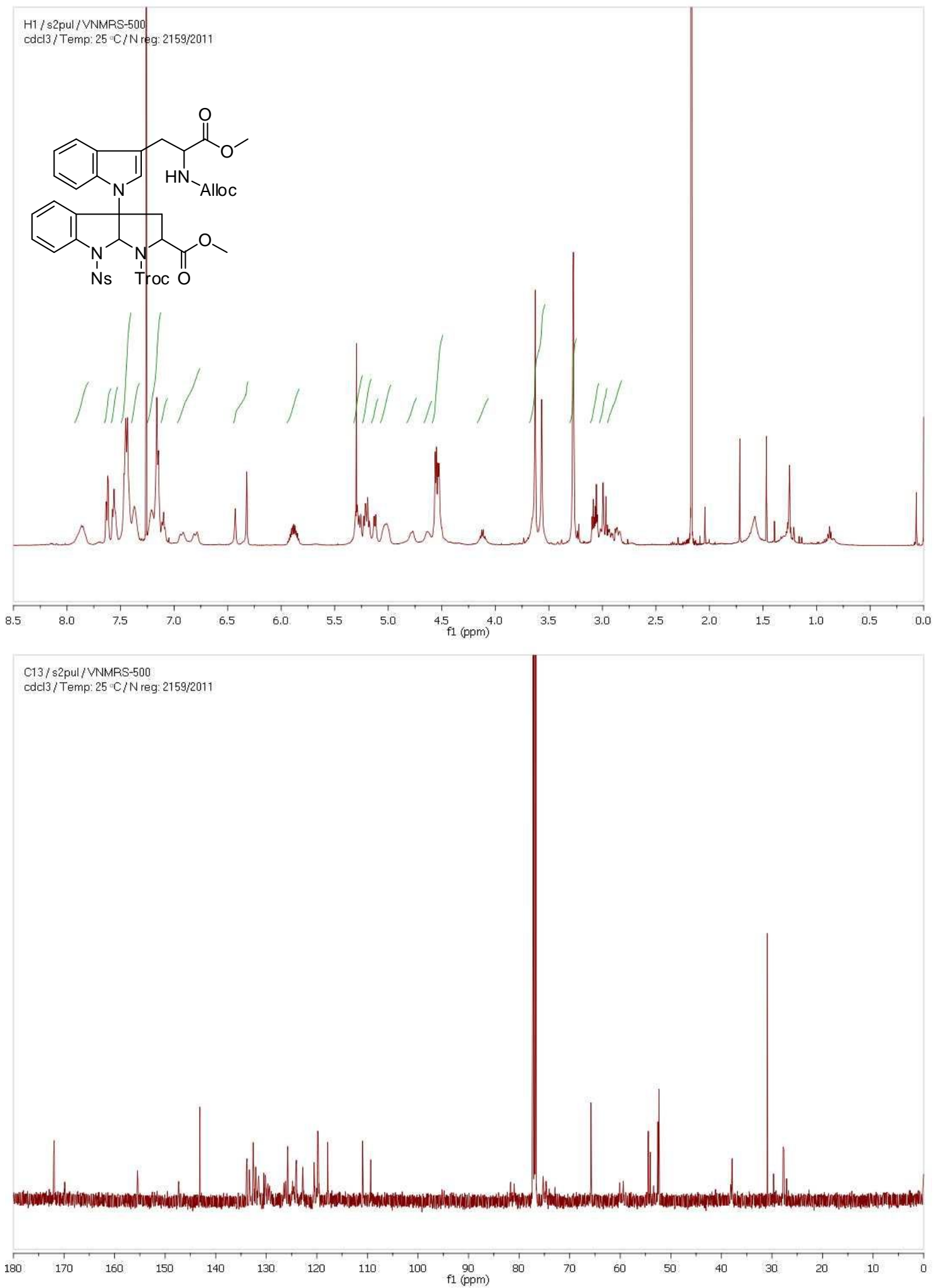
$1 f$.

H1/s2pul/Mercury-400

cdcl3/Temp: $25^{\circ} \mathrm{C} / \mathrm{N}$ reg: D-PCB190511091619
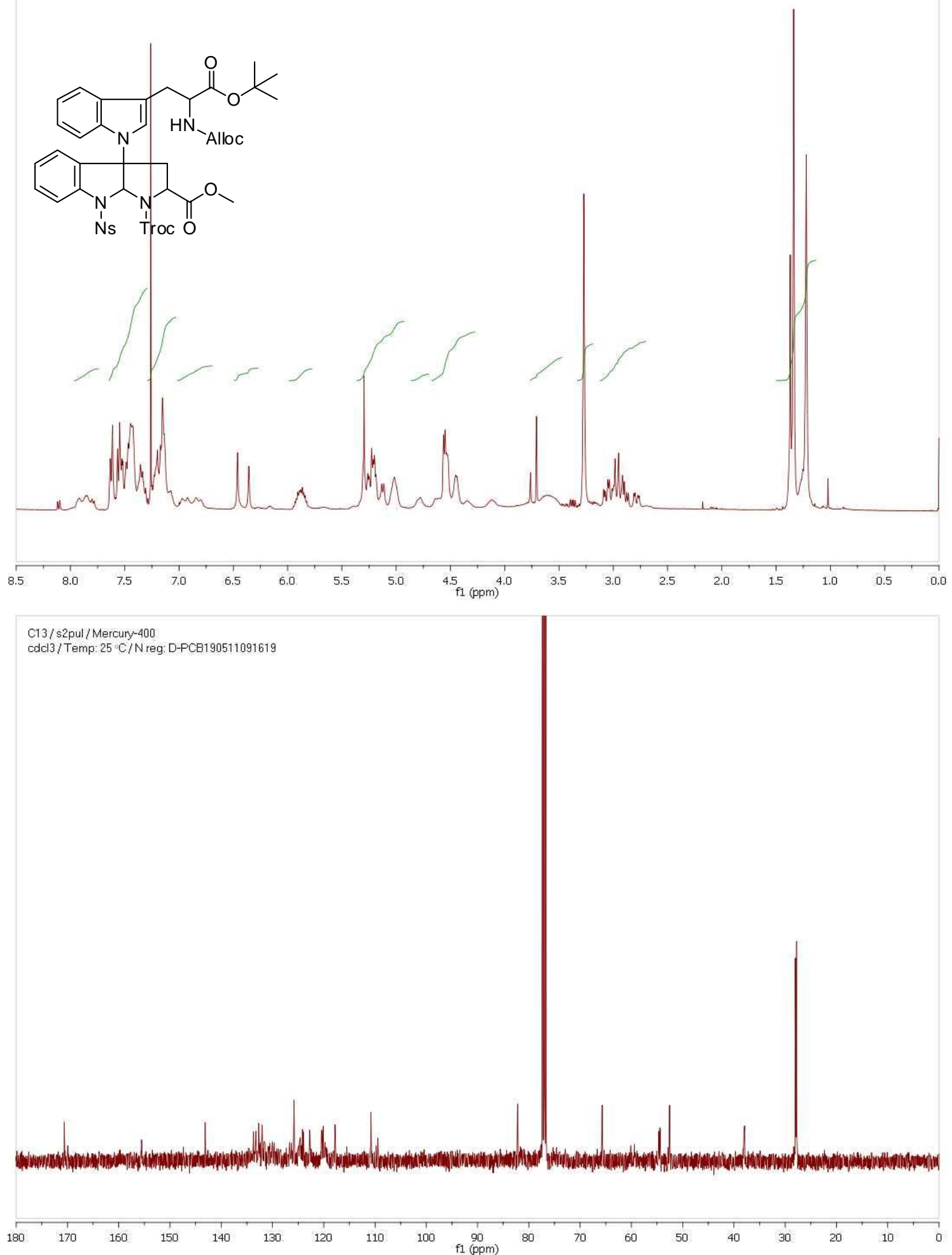
$1 \mathrm{~g}$.

H1/ s2pul/VNMRS-500

cdcl $3 /$ Temp: $25^{\circ} \mathrm{C} / \mathrm{N}$ reg: $2326 / 2010$<smiles>CCCCCNC(C(=O)NC(Cc1cn(C2(c3ccccc3P(=O)(O)O)CCC(C(=O)OC)N2C(C)(C)C)c2ccccc12)C(=O)OC(C)(C)C)C(C)CC</smiles>

$\mathrm{PhO}_{2} \stackrel{\mathrm{I}}{\mathrm{S}} \mathrm{M} \mathrm{oc} \mathrm{O}$

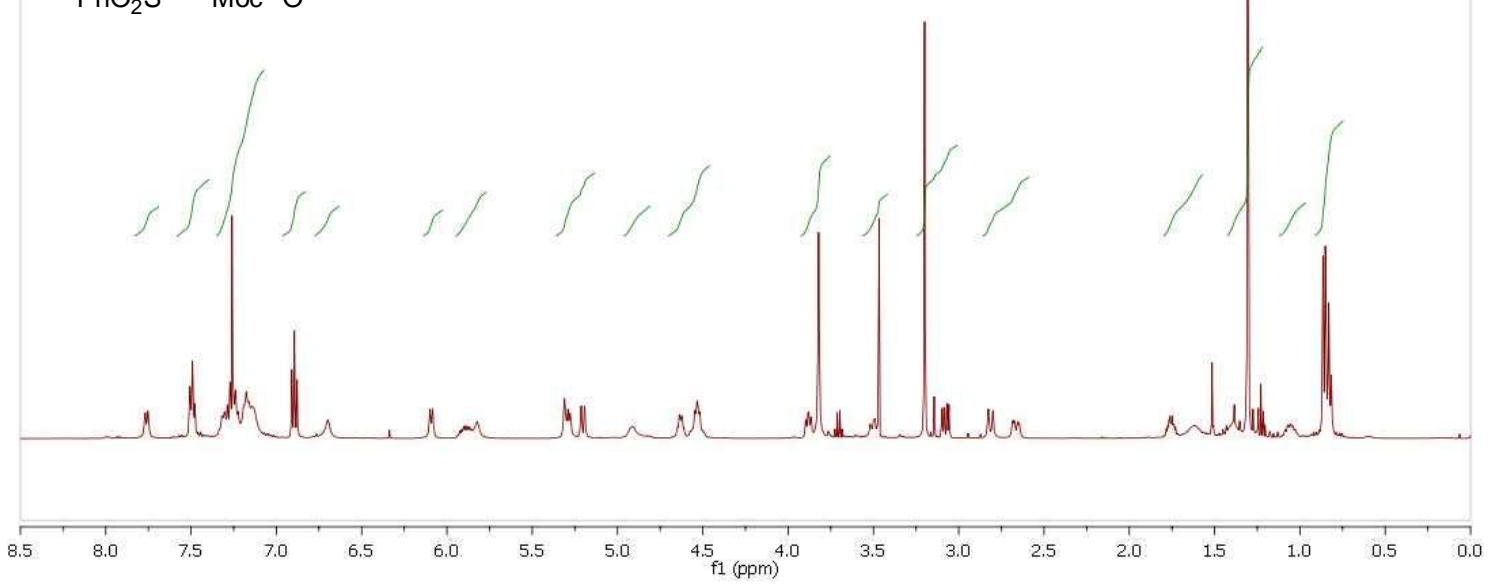

C13/s2pul/VNMRS-500

cdcl3/Temp: $25^{\circ} \mathrm{C} / \mathrm{N}$ reg: $2326 / 2010$

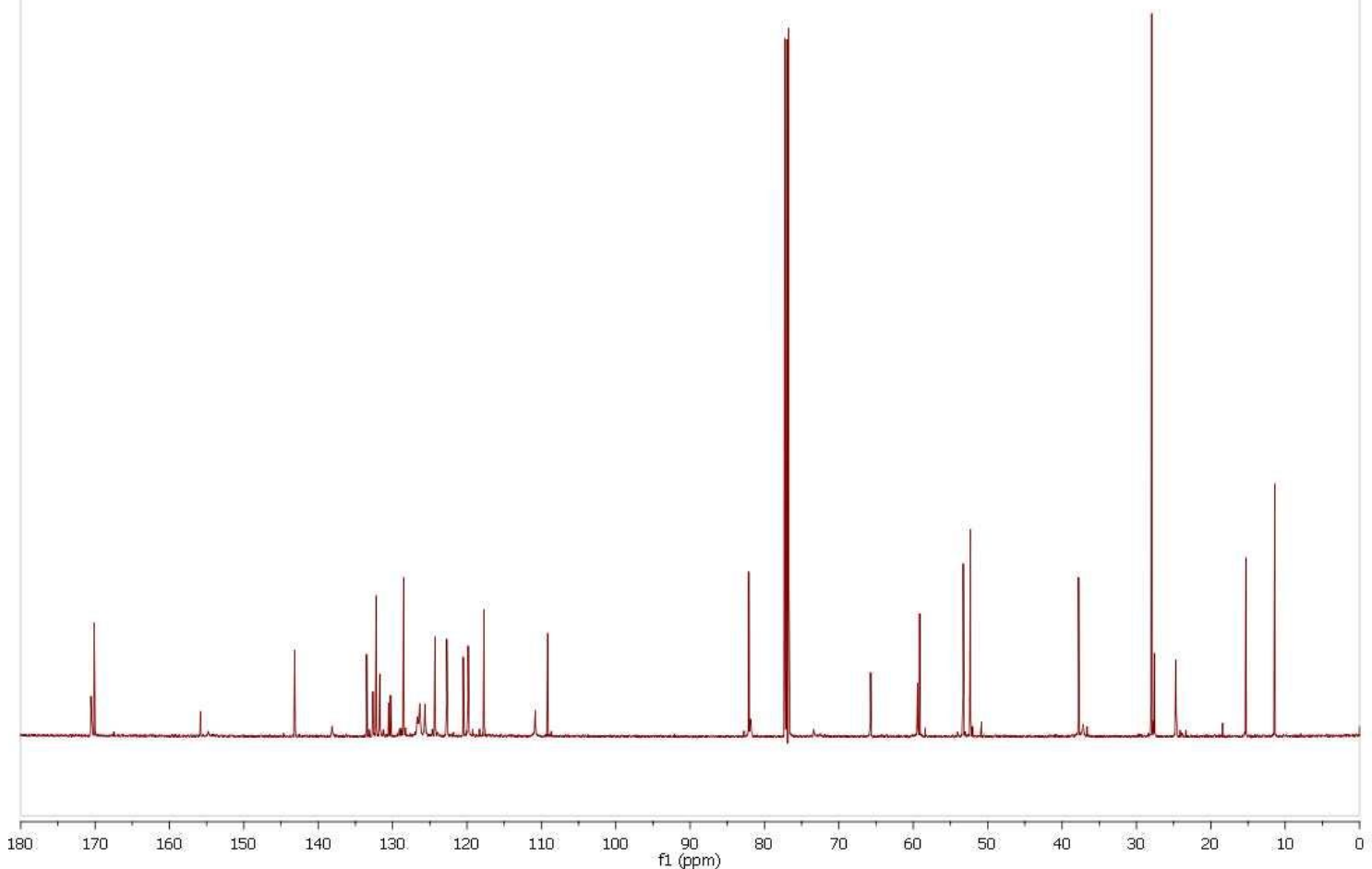


$1 h$.

H1 / s2pul/Mercury-400

Cdcl3/Temp $25^{\circ}$ / N reg: N-PCB170908093429<smiles>COC(=O)C(Cc1cn(C2(c3ccccc3N(S(=O)(=O)O)S(=O)(=O)O)CC(C(=O)OC)N(C)C2NC(C)=O)c2ccccc12)C(=O)OC</smiles>
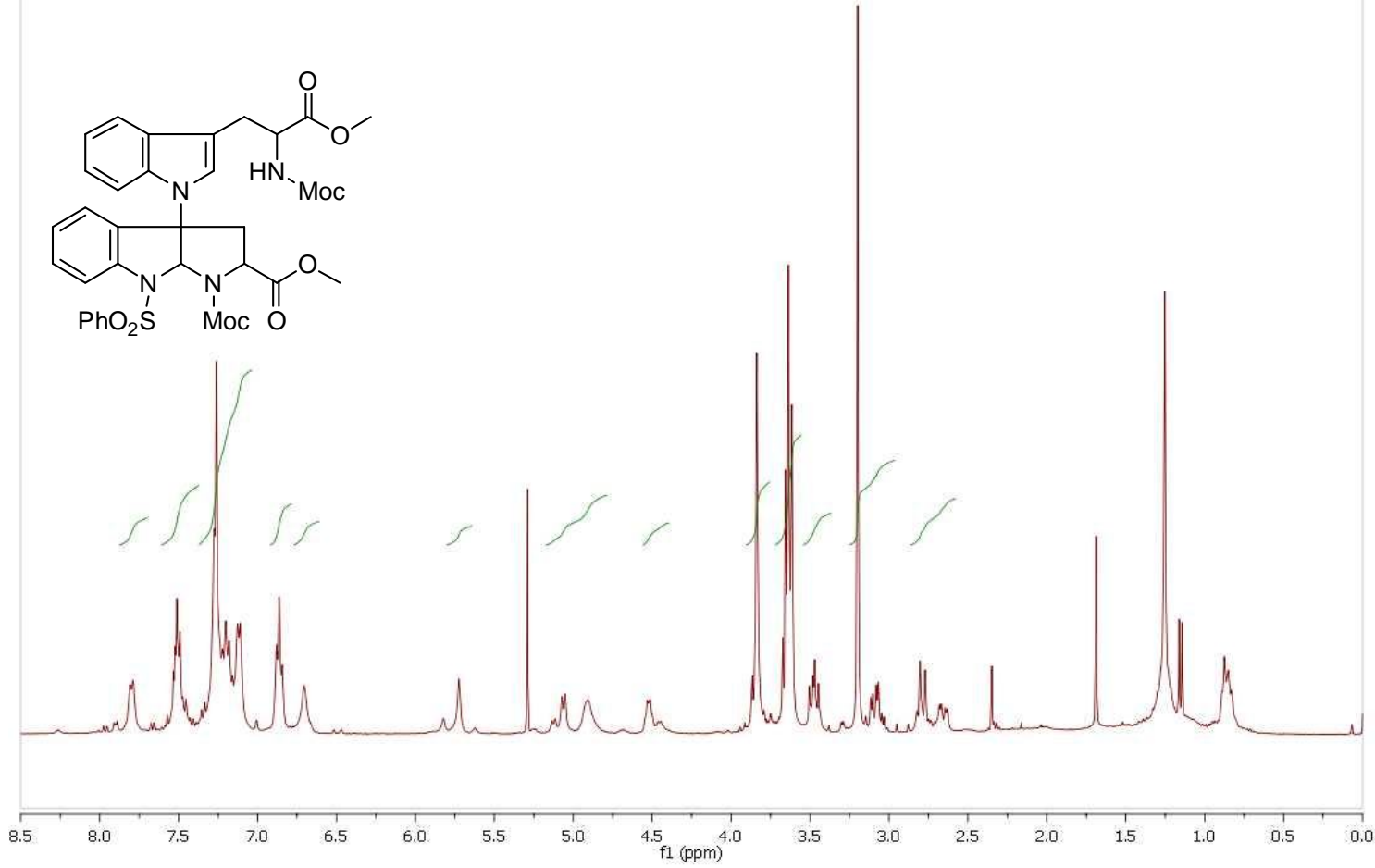

C13/s2pul/Mercury-400

cdcl3/Temp: $25^{\circ} \mathrm{C} / \mathrm{N}$ reg: N-PCB170908093429
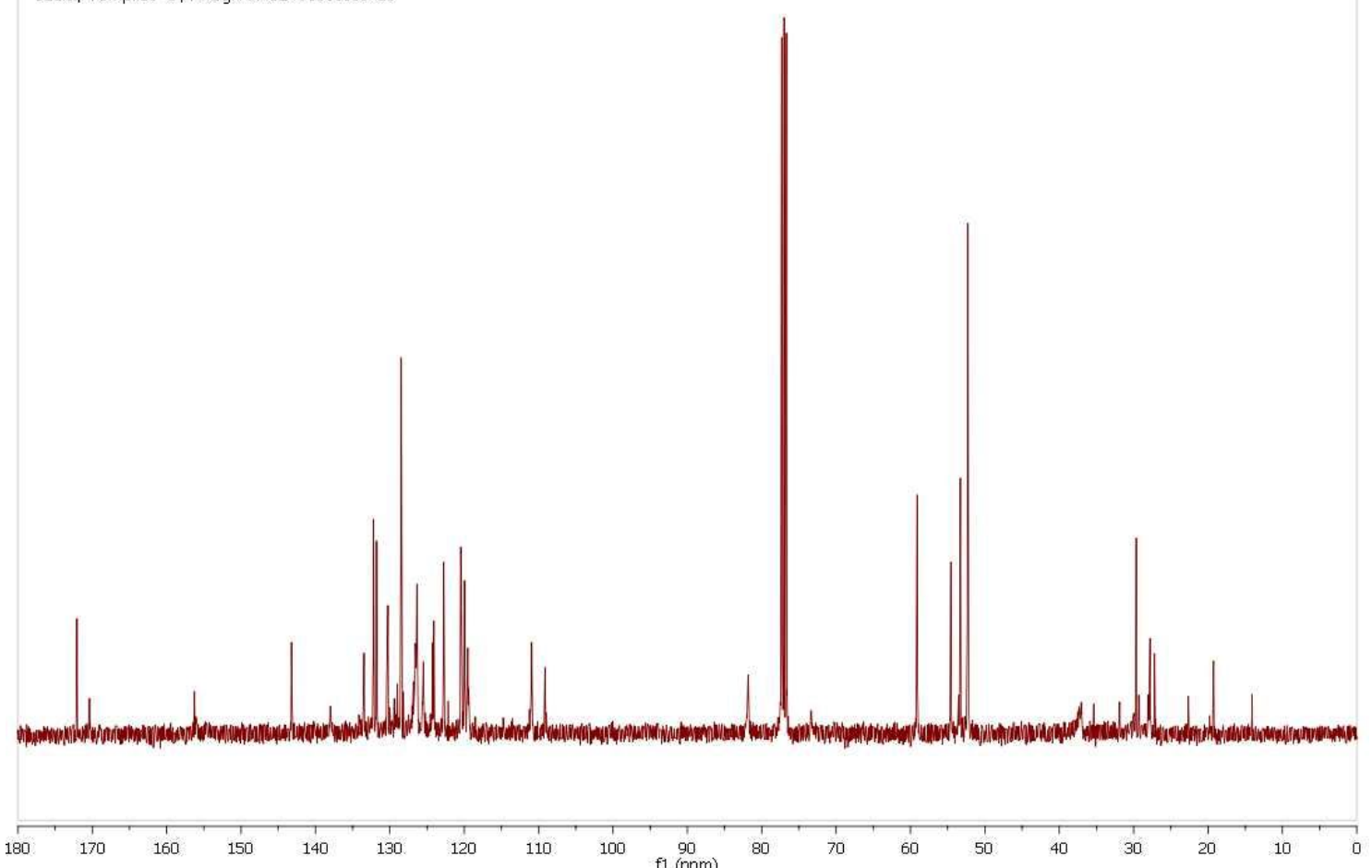
$1 i$.

$\mathrm{H} 1 /$ s2pul / Mercury-400

odd13/Temp: $25^{\circ} \mathrm{C} / \mathrm{N}$ reg: D-PCB221106090543
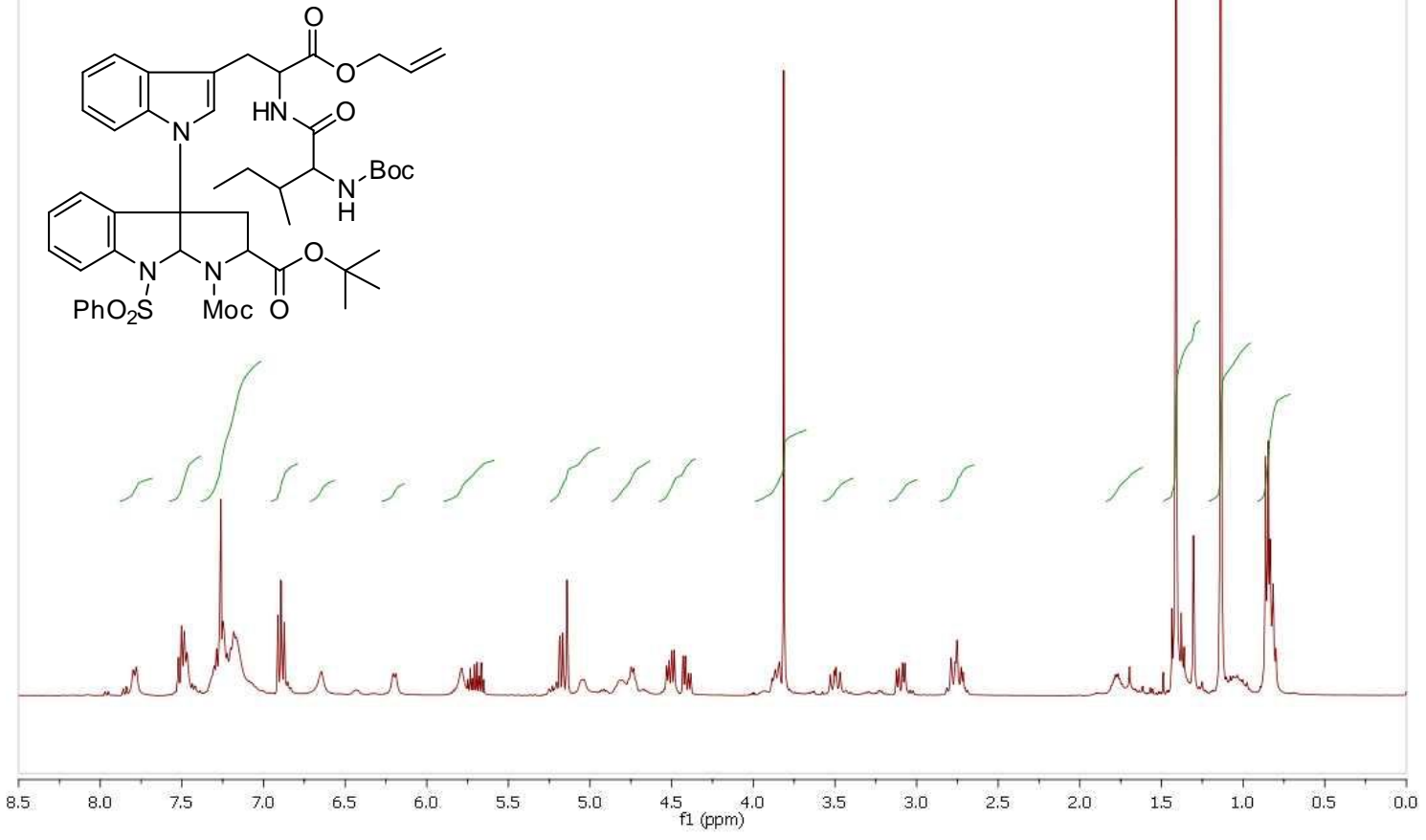

C13/s2pul/Mercury-400

cdcl3/Temp: $25^{\circ} \mathrm{C} / \mathrm{N}$ reg: D-PCB221206175924

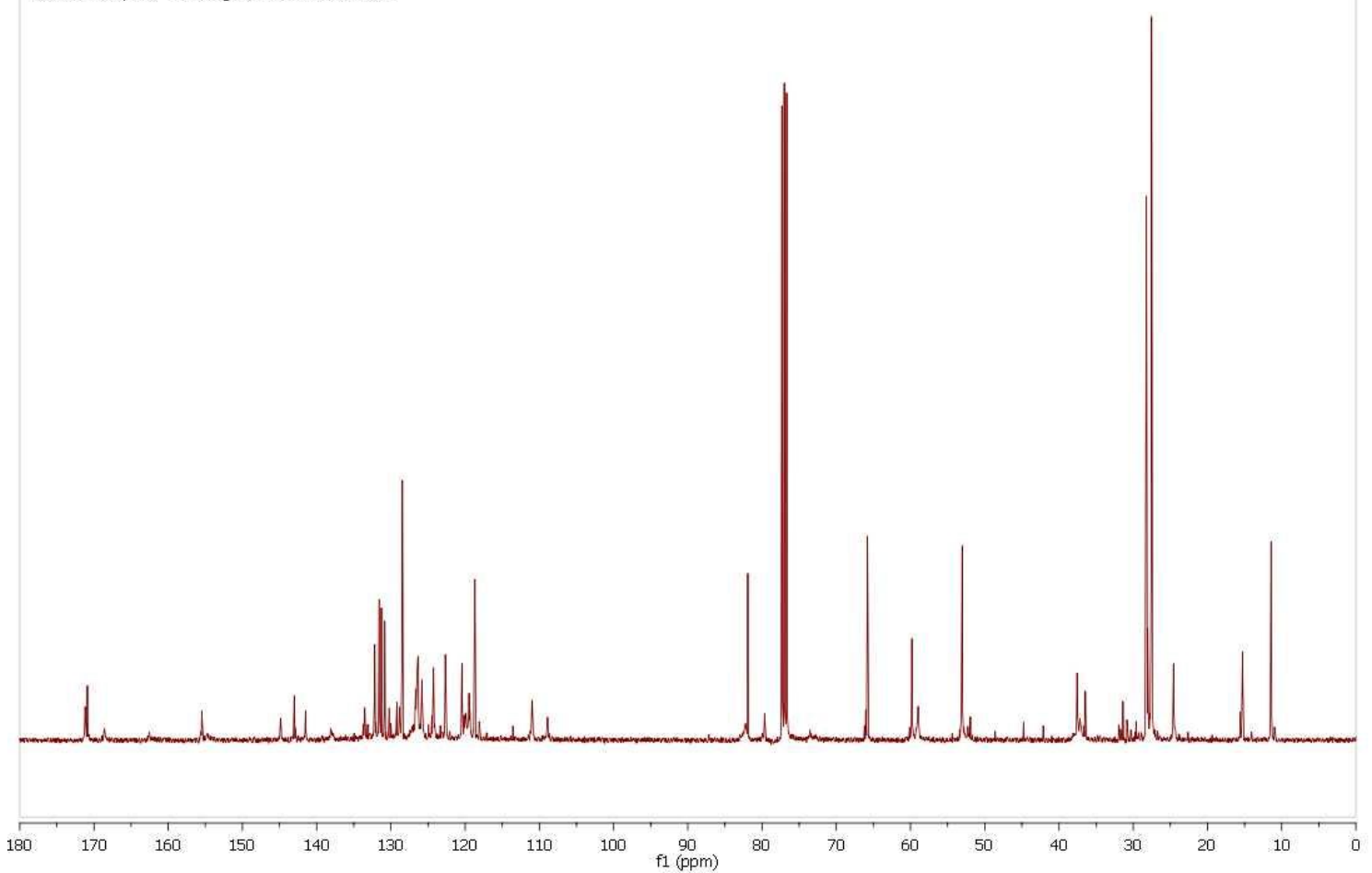


$1 \mathrm{j}$.

$\mathrm{H} 1 /$ s2pul/Mercuny-400

cdc13/Temp: $25^{\circ} \mathrm{C} / \mathrm{N}$ reg: N-PCB020609172323

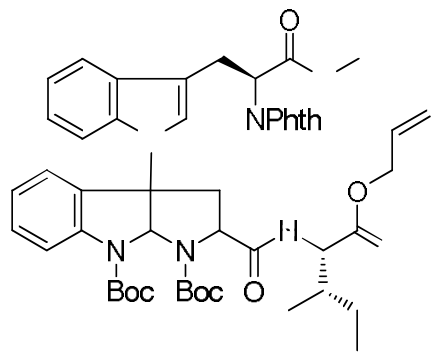
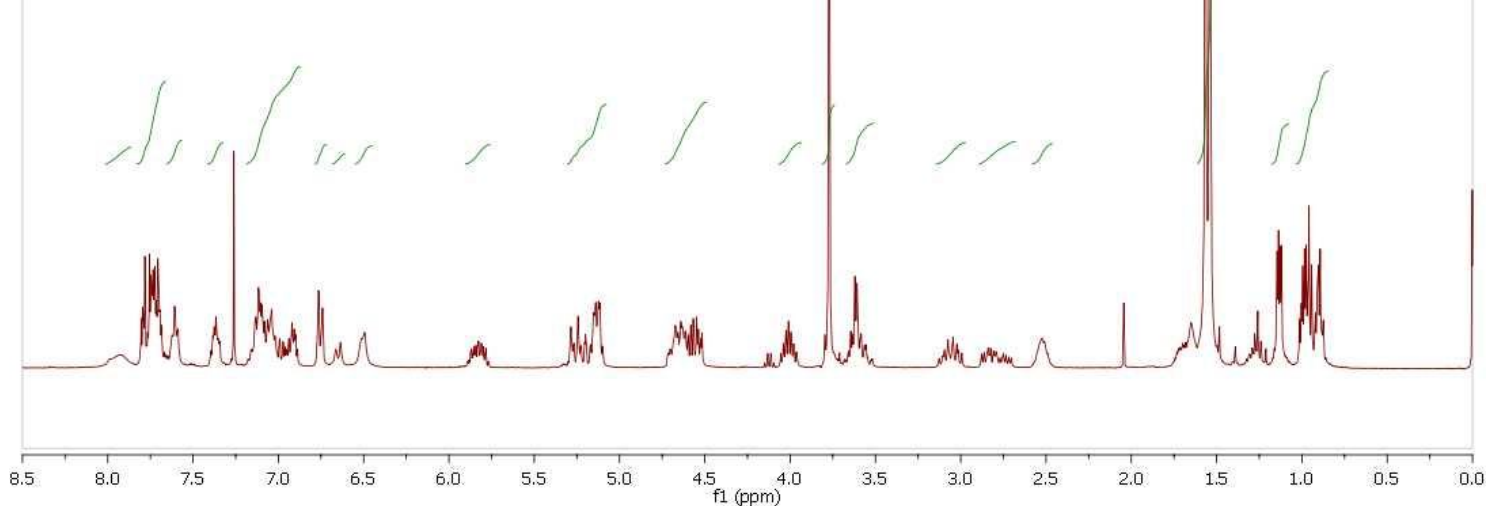

C13/s2pul/Mercury-400

cdc13/Temp: $25^{\circ} \mathrm{C} / \mathrm{N}$ reg: N-PCB020609172323
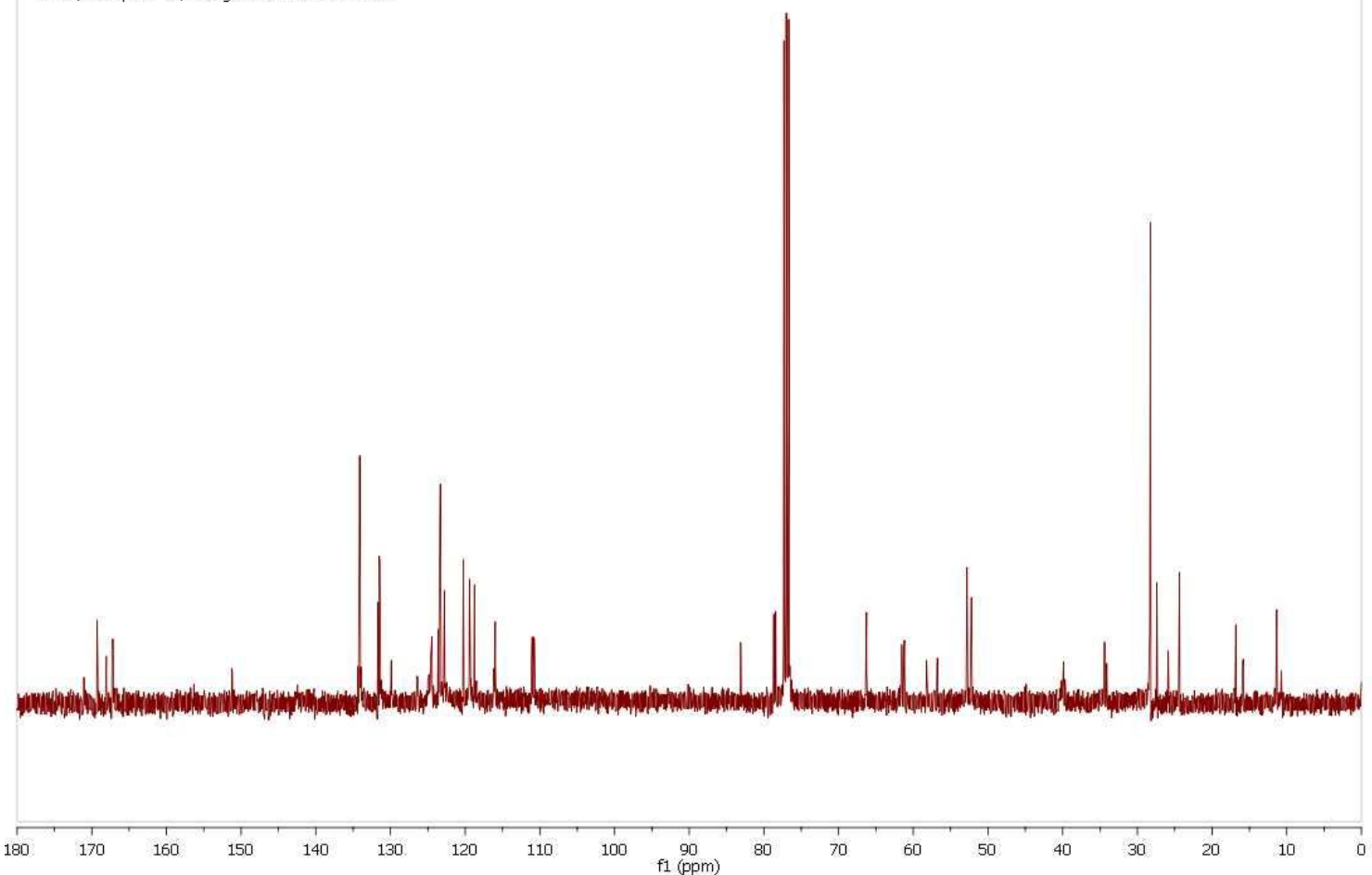
endo-3g.

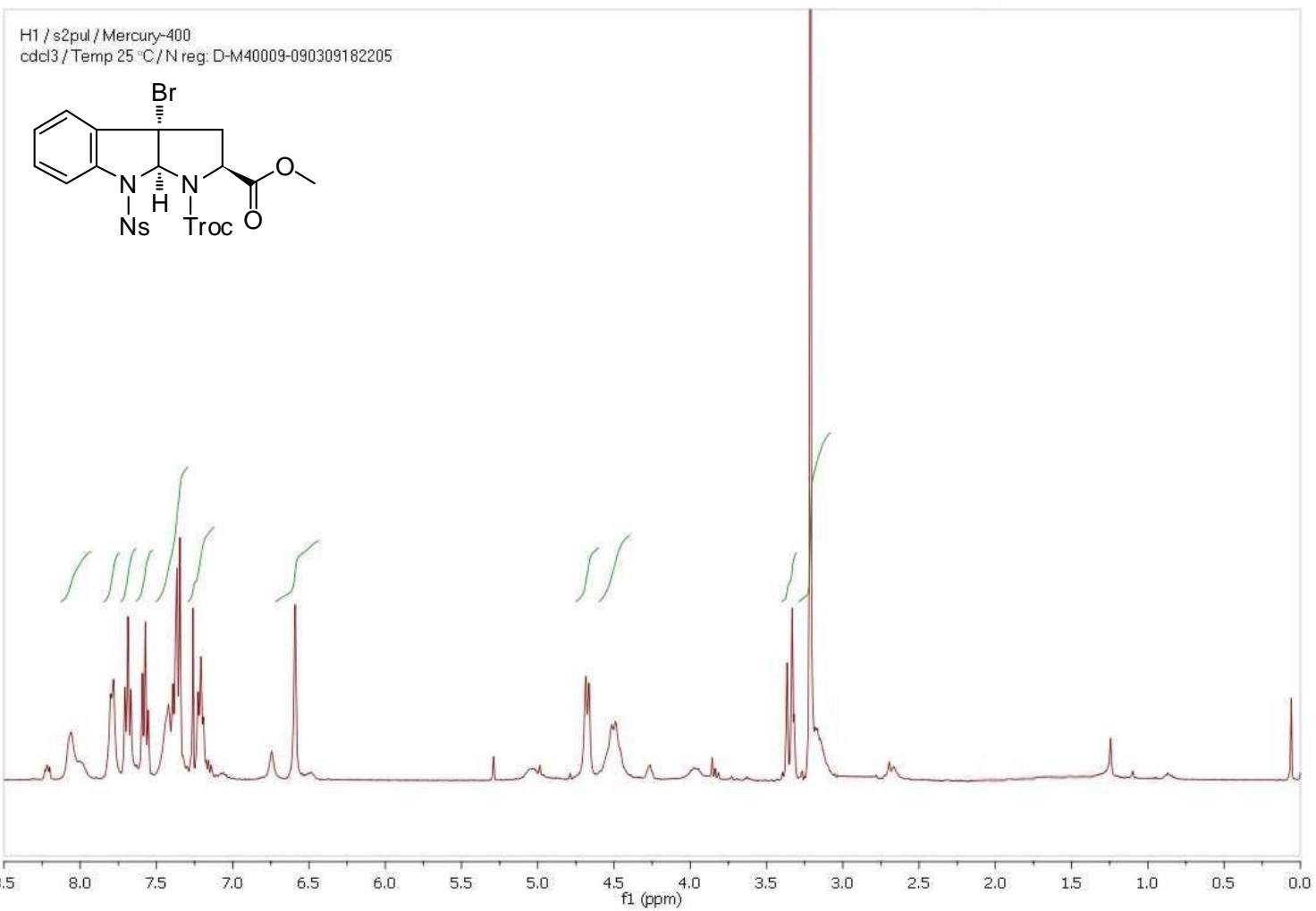

C13/s2pul/Mercury-400

cdcl3/ Temp $25^{\circ} \mathrm{C} / \mathrm{N}$ reg: D-M40009-090309182205

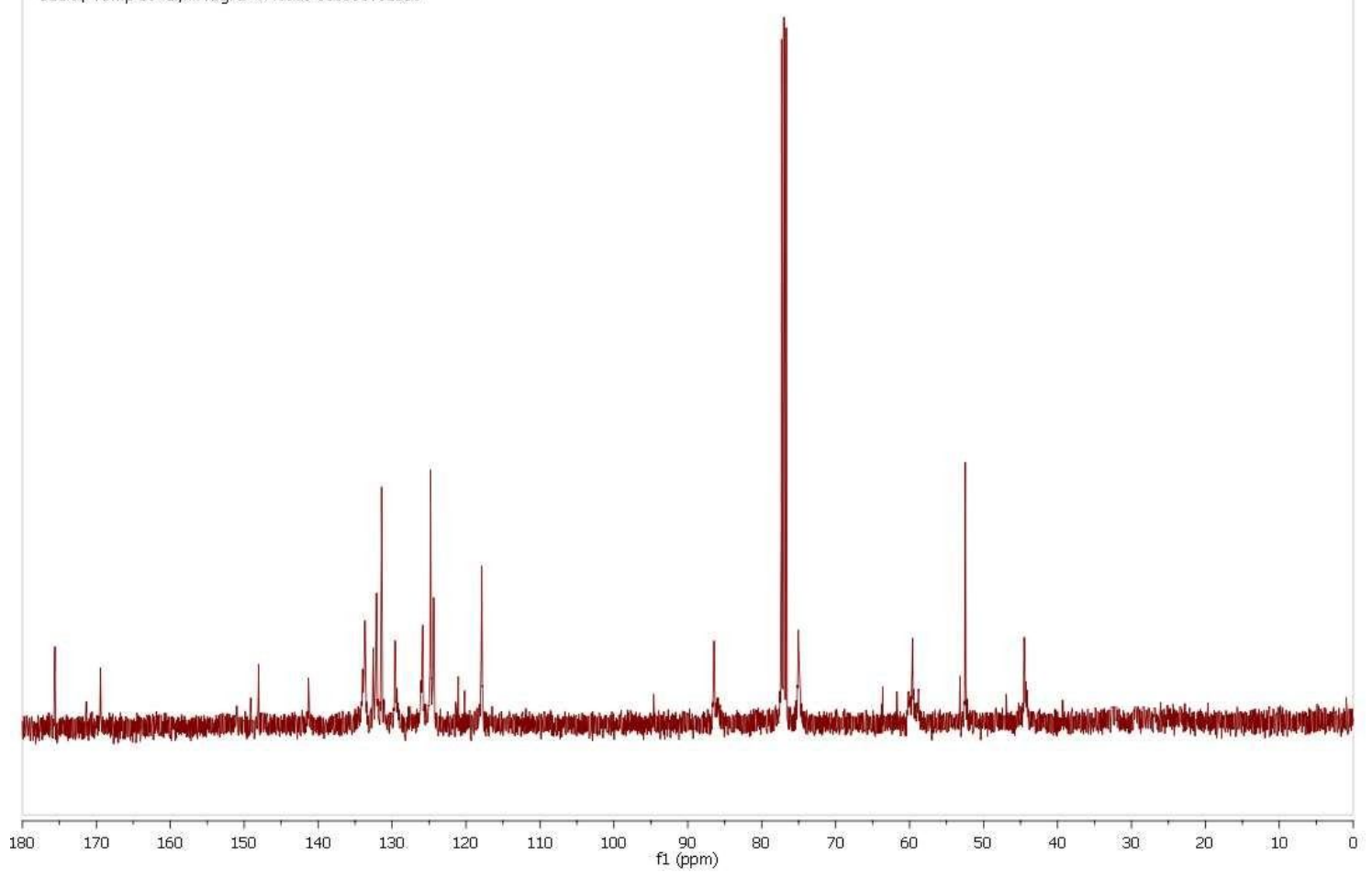


exo-3g.

H1/s2pul/Mercury-400

cdcl3/Temp $500^{\circ} \mathrm{C} / \mathrm{N}$ reg: 1160/2009
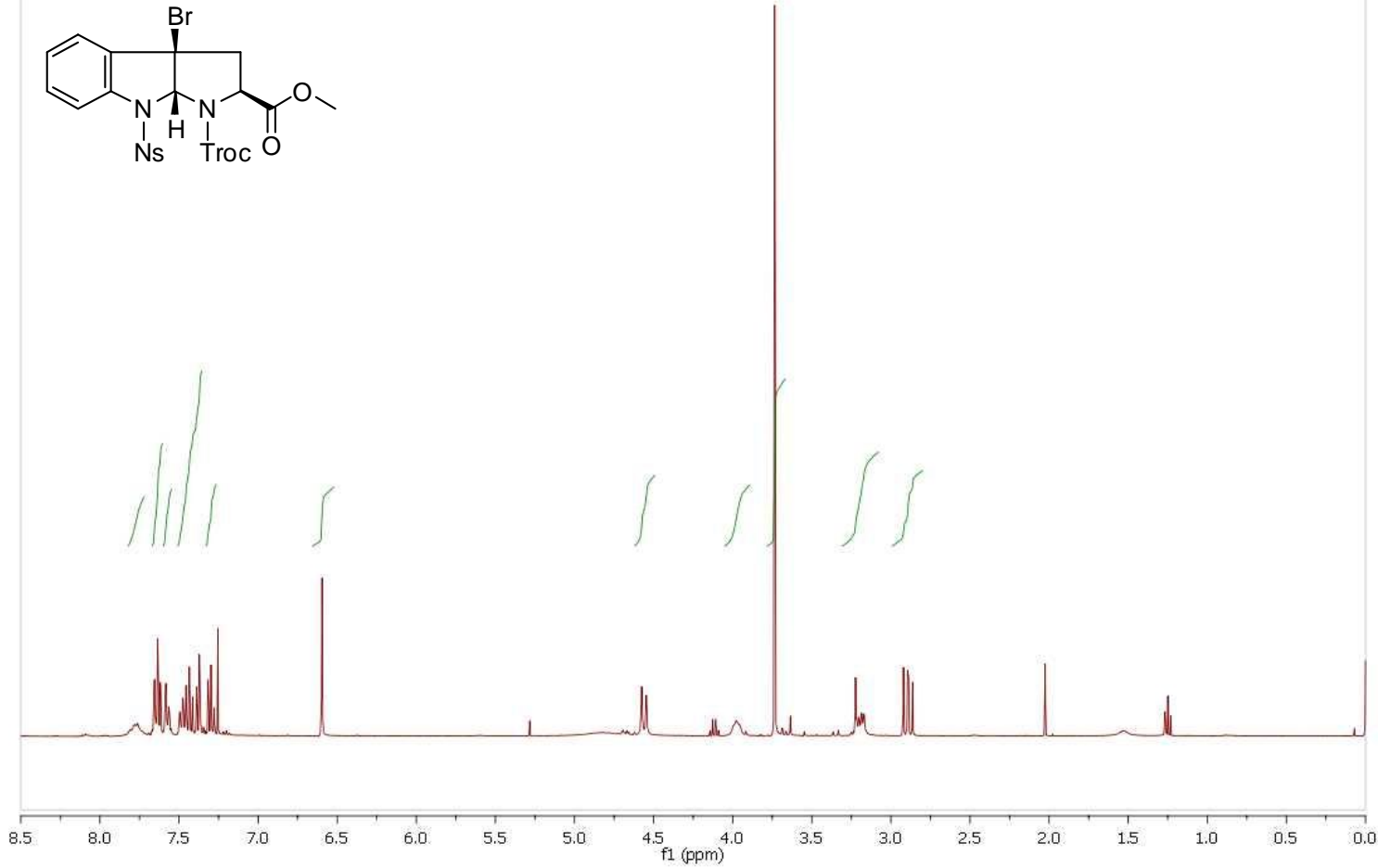

C13/s2pul/Mercury-400

cdcl3/Temp $50^{\circ} \mathrm{C} / \mathrm{N}$ reg: 1160/2009

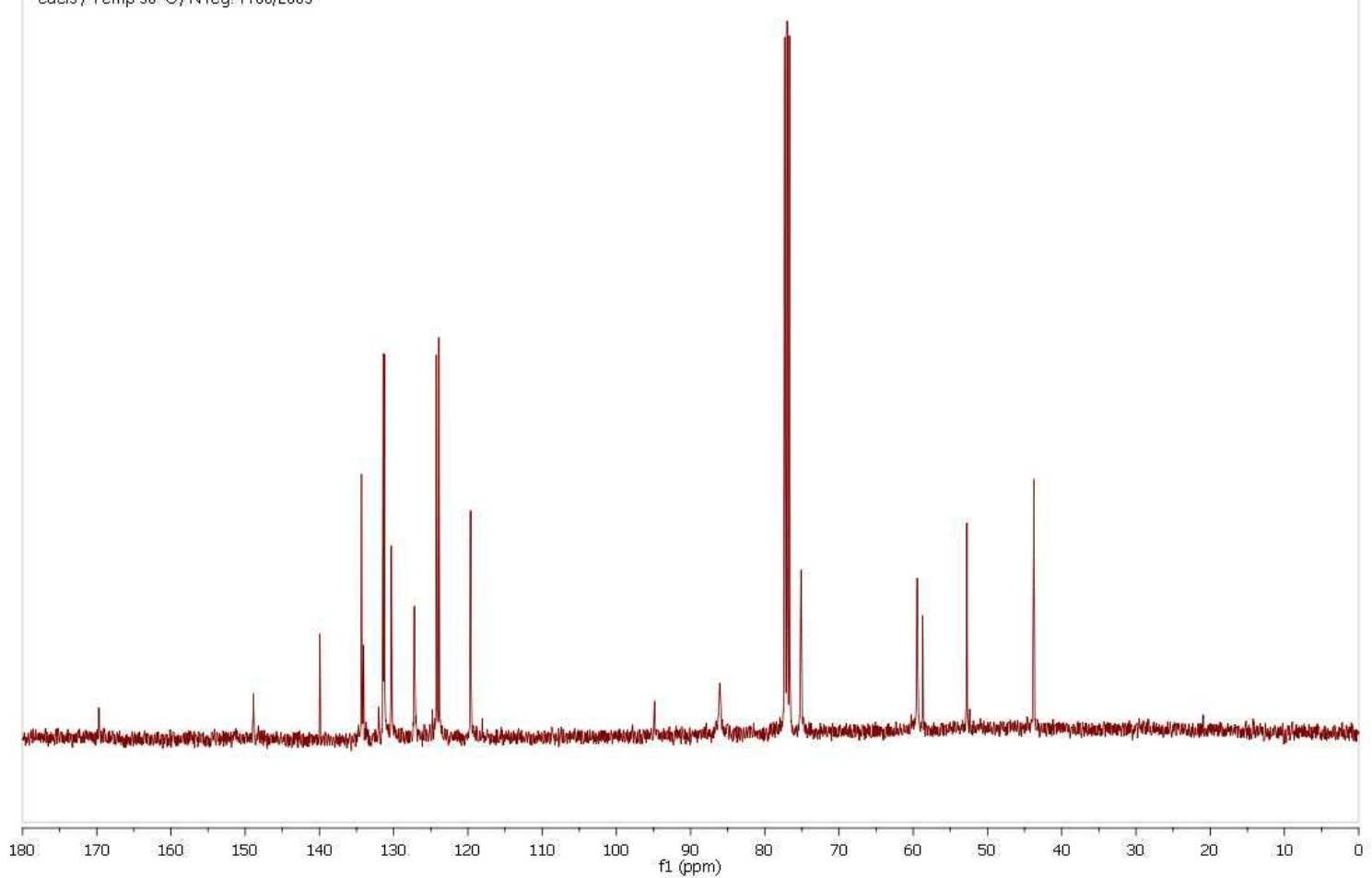




\section{Compost 5}
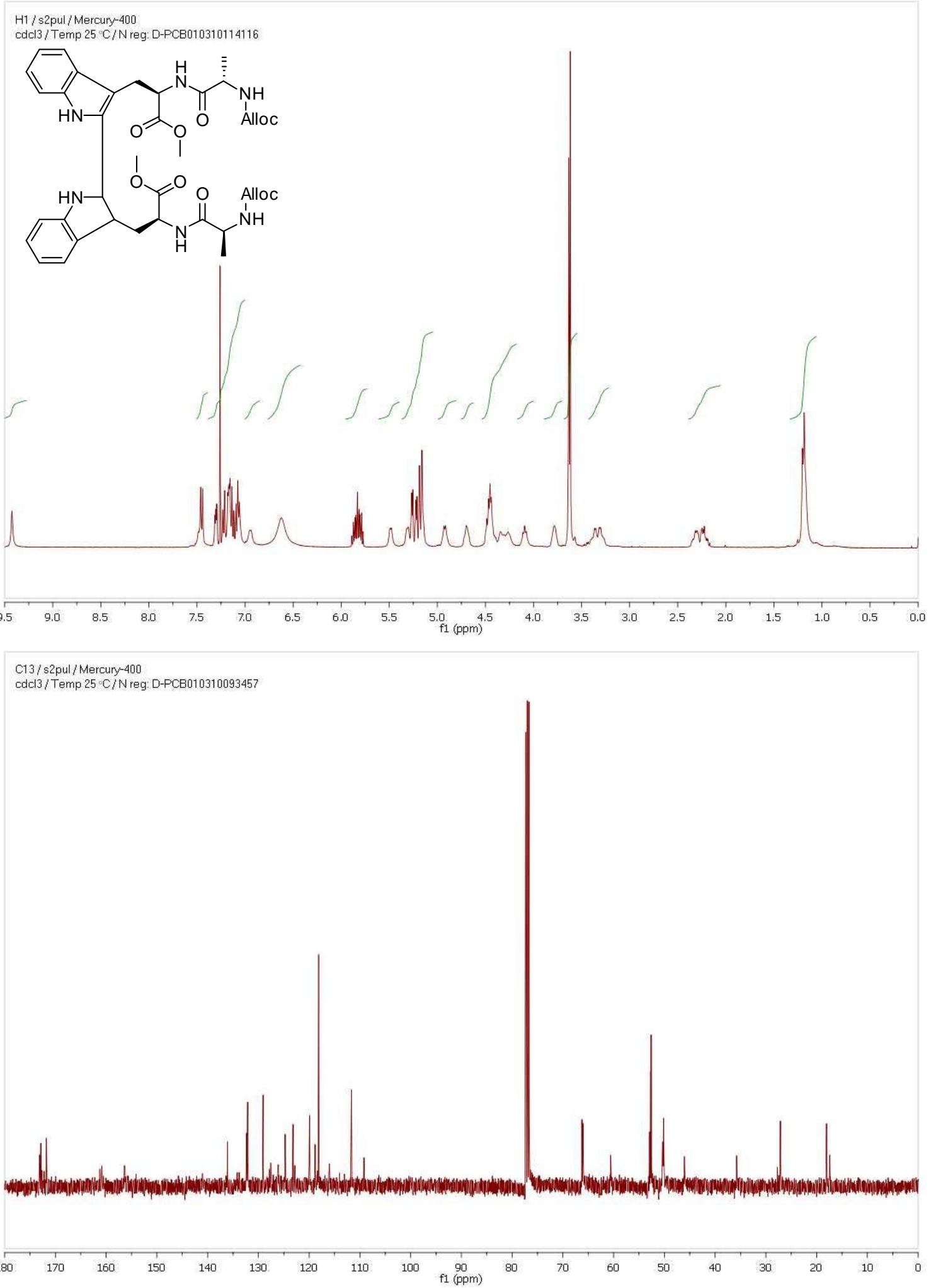


\section{Compost 8}

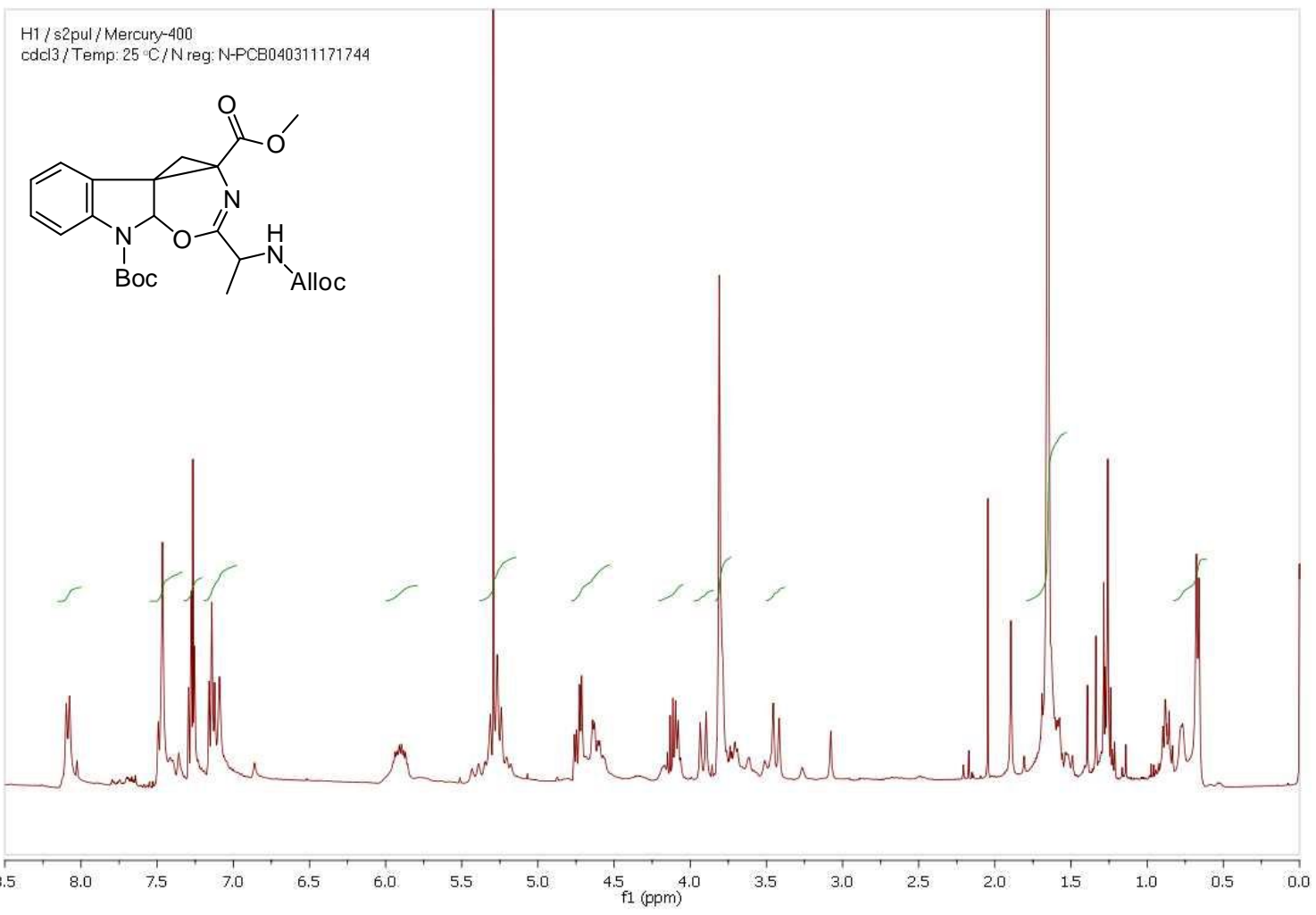

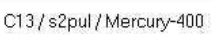

cdcl3/Temp: $25{ }^{\circ} \mathrm{C} / \mathrm{N}$ reg: N-PCB040311171744

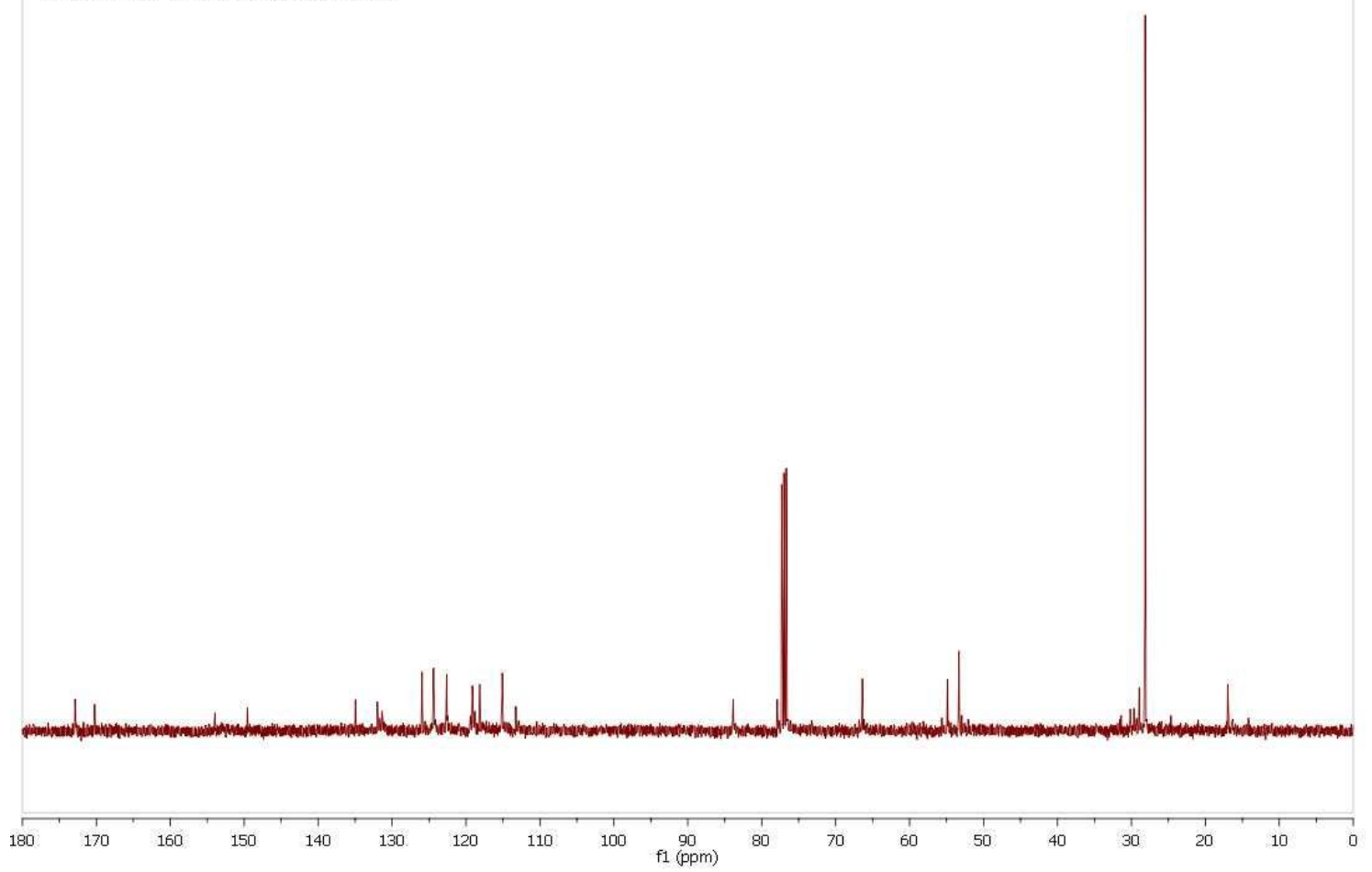



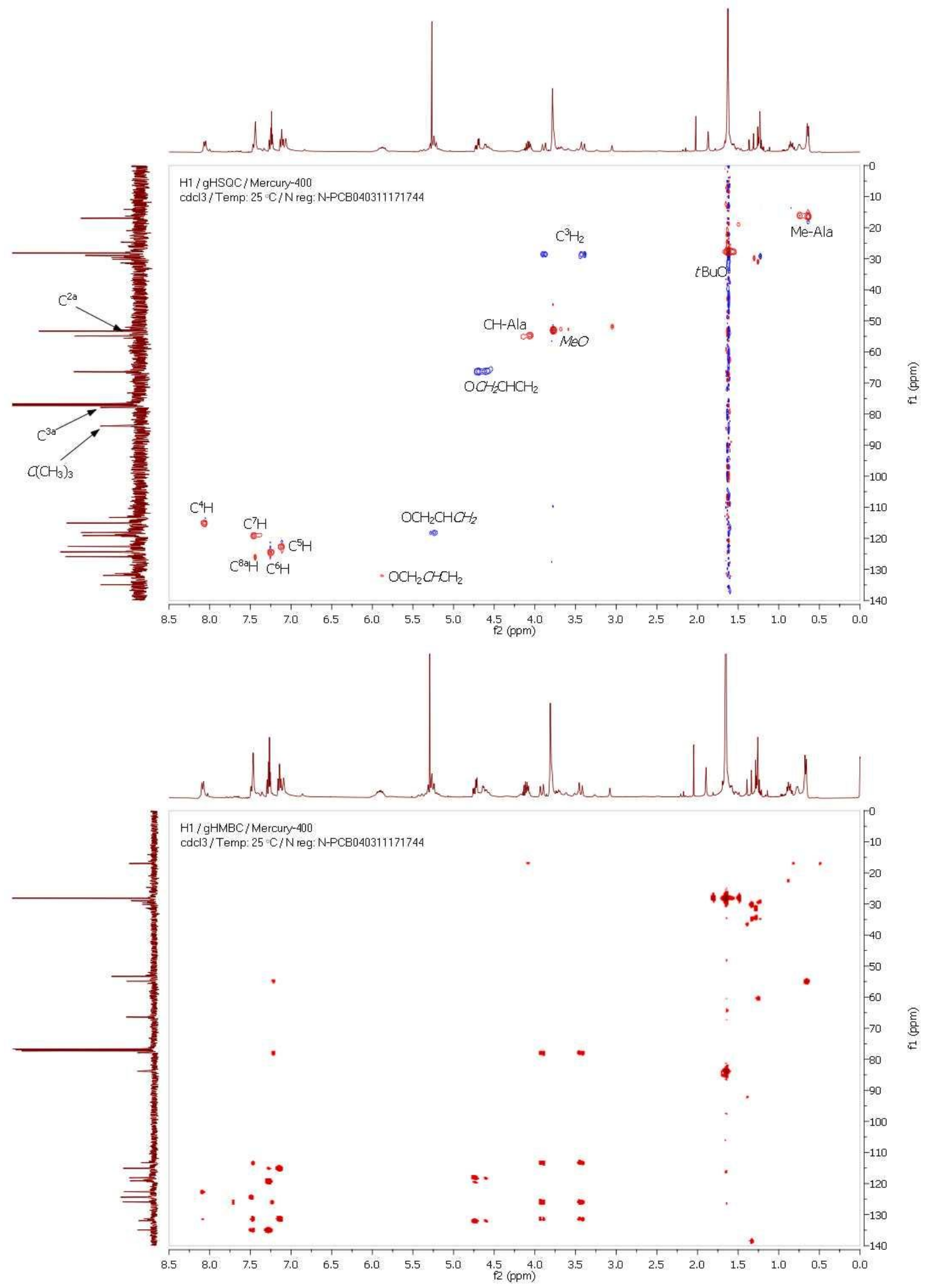